Tesis para obtener el grado de Doctor en Economía

\title{
Tópicos en el mercado inmobiliario
}

Un análisis desde la econometría espacial

\author{
Juan Ignacio Zoloa
}

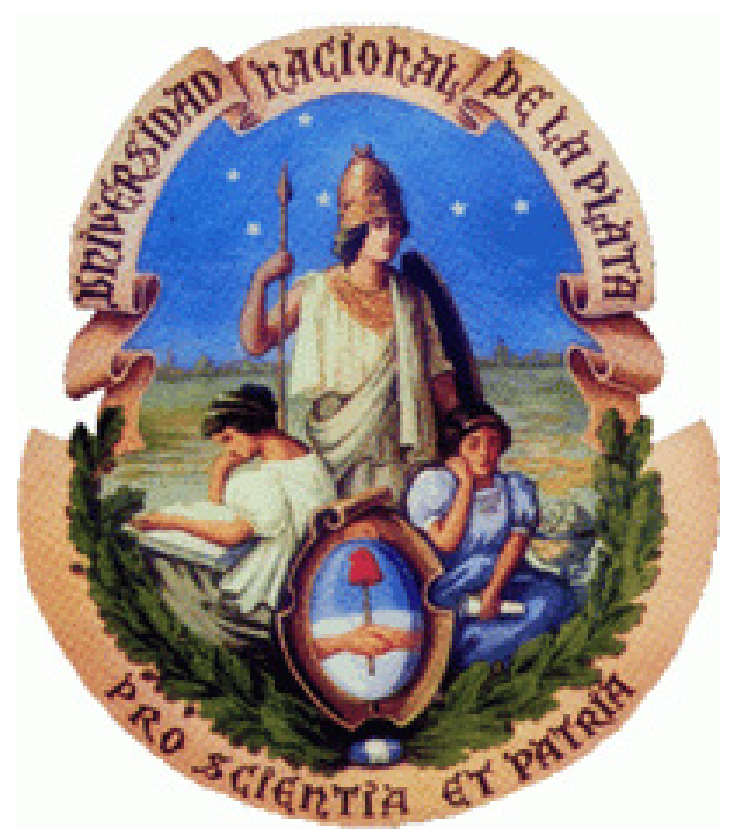

Universidad Nacional de La Plata

Facultad de Ciencias Económicas 


\section{Director}

Prof. Dr. Walter Sosa Escudero

\section{Co-Director}

Prof. Dr. Marcos Herrera Gómez 


\section{Agradecimientos}

Esta tesis no hubiera sido posible sin la colaboración de mucha gente, por eso quiero agradecer a mis directores, Walter y Marcos, por su generosidad, por su apoyo, por sus sugerencias y por su participación en el intercambio de ideas para el desarrollo de esta Tesis. A las autoridades del Comité de Doctorado por el aporte de ideas y de sugerencias a lo largo de los distintos seminarios. A mis compañeros de Doctorado y a los participantes de los seminarios por sus valiosos comentarios. A Leonardo Gasparini por sus sugerencias y por sus consejos a lo largo de todo el proceso. A Mariano Rabassa por los consejos y por las ideas que ayudaron a darle forma a este trabajo.

También aprovecho para agradecer a las personas que me empujaron (literalmente) en los primeros pasos de mi educación, a mi abuela, al abuelo, a mamá y, también, a papá. A Jorge y a Marta que fueron una influencia importante en los primeros tropiezos. A mis amigos, que estuvieron siempre. 



\section{Índice general}

$\begin{array}{lr}\text { Resumen } & 1\end{array}$

$\begin{array}{ll}\text { Introducción } & 3\end{array}$

$\begin{array}{ll}\text { 1. Modelos hedónicos espaciales } & 7\end{array}$

1.1. Introducción . . . . . . . . . . . . . . . . . . . 7

1.2. Modelo hedónico básico . . . . . . . . . . . . . . . . . 9

1.3. Modelos hedónicos espaciales . . . . . . . . . . . . . . . . . . 12

1.3.1. Detección de efectos espaciales . . . . . . . . . . . . . . 15

1.3.2. Especificación de modelos hedónicos espaciales . . . . . . . . . . . . 18

1.4. Estimación de los modelos hedónicos espaciales . . . . . . . . . . . . . . . 19

1.4.1. Modelo de rezago espacial (SLM) . . . . . . . . . . . . . . 20

1.4.2. Modelo de error espacial (SEM) . . . . . . . . . . . . . . . . 22

1.4.3. Modelo SARAR . . . . . . . . . . . . . . . . . 24

1.5. Interpretación de los resultados . . . . . . . . . . . . . 26

2. Valuación de los riesgos de inundación en La Plata 29

2.1. Revisión de la literatura . . . . . . . . . . . . . . . . . . . . 31

2.2. Datos utilizados . . . . . . . . . . . . . . . . . . 33

2.2.1. Mercado de Lotes baldíos . . . . . . . . . . . . . . . . 33

2.2.2. Riesgos de Inundación . . . . . . . . . . . . . . . . . . . . . 35

2.3. Estrategia empírica . . . . . . . . . . . . . . . . . . . 36

2.4. Resultados . . . . . . . . . . . . . . . . . . . 38

2.5. Conclusiones . . . . . . . . . . . . . . . . . . . . . 39

2.6. Apéndice . . . . . . . . . . . . . . . . . . . . . . 40

3. Valuación de la contaminación acústica en La Plata 47

3.1. Revisión de la literatura . . . . . . . . . . . . . . . . . . 48

3.2. Datos utilizados . . . . . . . . . . . . . . . . . . 49

3.2.1. Mercado de viviendas . . . . . . . . . . . . . . . . . . . . . . . . . 49

3.2.2. Contaminación acústica . . . . . . . . . . . . . . . 51

3.3. Estrategia empírica . . . . . . . . . . . . . . . . . . . 52 
3.4. Resultados . . . . . . . . . . . . . . . . . . . . . 53

3.5. Sensibilidad de los resultados . . . . . . . . . . . . . . . . 54

3.6. Conclusiones . . . . . . . . . . . . . . . . . . 57

3.7. Apéndice . . . . . . . . . . . . . . . . . . . 58

4. Valuación por cuantiles en la ciudad de Buenos Aires $\quad 71$

4.1. Regresiones espaciales por cuantil . . . . . . . . . . . . . . . 73

4.2. Datos utilizados . . . . . . . . . . . . . . . . . 75

4.3. Estrategia empírica . . . . . . . . . . . . . . . . 76

4.4. Resultados de la estimación . . . . . . . . . . . . . . . . . 77

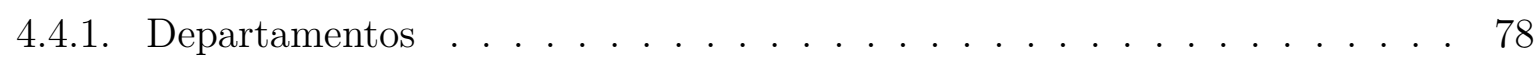

4.4.2. Casas . . . . . . . . . . . . . . . . . . . 79

4.4.3. Lotes baldíos . . . . . . . . . . . . . . . . 80

4.5. Conclusiones . . . . . . . . . . . . . . . . . . 80

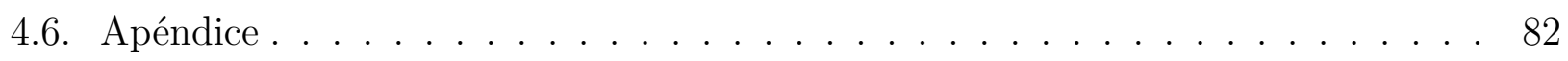

$\begin{array}{lr}\text { Consideraciones finales } & 97\end{array}$

$\begin{array}{ll}\text { Bibliografía } & 101\end{array}$ 


\section{Resumen}

Esta tesis doctoral aborda varios desafíos de investigación relacionados con el mercado inmobiliario. La primera línea de investigación está relacionada con los riesgos asociados a los desastres naturales; más precisamente, se estudia la valoración de los riesgos de inundación. Este problema se encuentra presente en muchas ciudades en desarrollo, en las cuales los individuos en pos de obtener lotes más baratos inician construcciones en lugares propensos a sufrir inundaciones. El segundo tópico se relaciona con la calidad del medio ambiente de las ciudades y analiza la valoración que realizan los individuos de vivir en zonas más tranquilas, con menos contaminación acústica. La última línea de trabajo analiza el hecho de que diversas características de los inmuebles pueden ser valoradas de una manera distinta a través de la distribución de precios; más precisamente, se analiza si el valor marginal de una determinada característica puede ser diferente dependiendo del lugar en la distribución de precios.

Los mencionados tópicos son abordados utilizando los avances más recientes en las técnicas econométricas. Las estimaciones realizadas introducen interacciones espaciales que permiten capturar el impacto del vecindario geográfico sobre cada inmueble observado, así como mejorar la precisión de los parámetros considerando la posible presencia de heteroscedasticidad en el término de error.

La principal motivación de esta tesis es contribuir a una mejor medición de la valuación que hacen los individuos de algunas características ambientales y de las diversas características de los inmuebles. En algunos de los temas analizados, existe una amplia literatura; sin embargo, la misma se focaliza casi por completo en mercados inmobiliarios de países desarrollados. La evidencia empírica proveniente de países en desarrollo es relativamente escasa y, en el caso particular de la Argentina, casi no se observan estudios sobre dichos temas.

Una de las contribuciones de esta tesis es la estimación de la valoración de los riesgos de inundación en la ciudad de La Plata. El análisis indica que cuando las propiedades se encuentran en zonas con alto riesgo hídrico sufren un importante descuento en el precio. Dicho descuento llega, aproximadamente, al $12 \%$ en las zonas propensas a inundaciones.

Por otra parte, las conclusiones del análisis de las condiciones medioambientales ponen de relieve la importancia que la contaminación acústica tiene para los habitantes de la ciudad de La Plata, en la cual un comprador estaría dispuesto a pagar alrededor de un 1,9\% menos por cada decibel. 
Finalmente, el análisis de las valoración de las características a través de la distribución de precios revela la existencia de una variación sustancial de varios atributos a través de la distribución de precios inmobiliarios.

Las valoraciones obtenidas en esta tesis servirán para mejorar la eficacia de las políticas públicas, las cuales tienen como objetivo mejorar la calidad de vida de las personas, como son la legislación sobre el uso del suelo, el planeamiento urbano y la aplicación de regulaciones sobre la contaminación ambiental. Es importante remarcar que uno de los aspectos más relevantes en la evaluación de dichas políticas radica en la comparación de los beneficios económicos obtenidos por estas acciones respecto a los costos incurridos en aplicar tales políticas. Esta tesis permite contar con medidas cuantitativas apropiadas del valor económico de los beneficios esperados y es en este punto donde se realiza el principal aporte. 


\section{Introducción}

El mercado inmobiliario es de central interés para la determinación del nivel de bienestar de la sociedad y para el nivel de actividad económica. En una revisión realizada por Muellbauer \& Murphy (2008) puede observarse cómo el mercado inmobiliario presenta múltiples interacciones con el resto de los sectores económicos e impacta en el ingreso, en las tasas de interés, en la disponibilidad crediticia y afecta, también, los patrones migratorios y demográficos. La importancia de este mercado ha sido tal que numerosos economistas se han dedicado al análisis de su funcionamiento y de su estructura. A su vez, especial atención ha sido puesta sobre el impacto en el mercado inmobiliario de determinadas características y de factores ambientales. Esta tesis intenta acercar al lector los últimos avances en los métodos empíricos para valorar adecuadamente dichos factores.

La primera línea de investigación está relacionada con los riesgos asociados a los desastres naturales; más precisamente, se estudia la valoración de los riesgos de inundación. Este problema se encuentra presente en muchas ciudades en desarrollo, en las cuales los individuos, en pos de obtener lotes más baratos, inician construcciones en lugares propensos a sufrir inundaciones $^{1}$. El segundo tópico se relaciona con la calidad del medio ambiente de las ciudades y analiza la valoración que realizan los individuos de vivir en zonas más tranquilas, en términos de contaminación acústica. La última línea de trabajo analiza el hecho de que diversas características de los inmuebles pueden ser valoradas de manera distinta a lo largo de la distribución de precios, como así también pueden estar influenciadas por componentes espaciales. Más precisamente, se analiza cómo el valor marginal de una determinada característica puede ser diferente dependiendo del lugar en la distribución de precios.

Una característica común de los temas de investigación analizados es la inexistencia de un mercado directamente observable. Debido a esta particularidad, la asignación de un valor a las características estudiadas se convierte en una tarea difícil, ya que este valor no se captura en las transacciones que se realizarían regularmente en un mercado explícito. Para salvar esta dificultad, la literatura ha propuesto tres alternativas que permiten obtener estos valores (Freeman III, 2003).

\footnotetext{
${ }^{1}$ Además de zonas inundables, los individuos de menores recursos tienden a instalarse en zonas cercanas a plantas químicas, a basurales de desechos urbanos y descartes tecnológicos, en la rivera de los lugares donde las fábricas y principalmente las curtiembres arrojan sus desechos.
} 
La primera alternativa es el método de valuación contingente que consiste en preguntar directamente a la persona cuánto estaría dispuesta a pagar ante una modificación en alguna característica del inmueble o de la zona. Por ejemplo, en el caso de la contaminación acústica, se le pediría que declare su disposición a pagar ante una reducción del ruido.

Otra valoración alternativa es el método de gastos defensivos (averting behavior), el cual consiste en contabilizar los gastos que una persona realiza para reducir su exposición a la amenidad. En el caso de los riesgos de inundación, consistiría en sumar los gastos en compuertas que impidan la entrada de agua al inmueble durante las inundaciones.

Finalmente, el tercer método es el enfoque de preferencias reveladas que consiste en considerar las decisiones que los individuos realizan en otros mercados y de ahí extraer la valoración de cada una de las características. La idea es identificar las preferencias observando las elecciones que realizan los consumidores entre diferentes productos o características en mercados vinculados. Estas elecciones revelan las preferencias de los individuos permitiendo cuantificar cuanto están dispuestos a pagar por cada característica o atributo.

En esta tesis la estrategia utilizada es una derivación del enfoque de las preferencias reveladas conocida como modelo de precios hedónicos. Desde el trabajo pionero de Lancaster (1966) y Rosen (1974), muchos estudios han aplicado el modelo de precios hedónicos para conocer la relación entre los precios de los inmuebles, las características constructivas y los riesgos medioambientales, tales como inundaciones, terremotos o la cercanía a plantas químicas.

La formulación más general de un modelo hedónico relaciona el precio de un bien con sus atributos. En particular, si la función de utilidad de un individuo se define como una función de las características del inmueble, la calidad ambiental, las características socioeconómicas de la zona y de un bien compuesto, la maximización de su utilidad llevaría al individuo a igualar la utilidad marginal de cada uno de los atributos con sus precios marginales. La derivada de la ecuación de precios hedónicos con respecto a cada variable explicativa es su precio marginal implícito. Este precio marginal puede interpretarse como la disposición marginal a pagar asumiendo que el mercado inmobiliario se encuentra en equilibrio. Además, el modelo hedónico sugiere que los beneficios derivados de una modificación en alguna de las características, como ser una reducción en el riesgo de inundaciones o una disminución en el nivel de contaminación acústica, se reflejará en el precio que los individuos pagan en el mercado por la propiedad que elijen. En otras palabras, el fundamento de este enfoque es que, ceteris paribus, la propiedad en una zona con menor riesgo de inundación o con menos contaminación acústica tendrá estos beneficios capitalizados en su valor, el cual debería reflejarse en unos precios más altos.

Aunque los modelos hedónicos tienen una larga historia como herramientas para la valoración económica de los bienes que no tienen un mercado particular, sólo recientemente han introducido la dimensión espacial dando lugar a los denominados modelos hedónicos espaciales. La introducción de espacio en los modelos hedónicos presenta desafíos adicionales, incluyendo 
nuevos métodos de estimación y una interpretación alternativa de los resultados teóricos y empíricos.

Si bien se conocen las implicacias teóricas de la omisión de las interacciones espaciales, sin la realización de pruebas de diagnóstico y la estimación de los modelos pertinentes, es imposible determinar si la falta de consideración de las interacciones espaciales tiene un efecto significativo en las estimaciones. Sólo mediante aplicaciones empíricas puntuales se puede determinar el impacto que generan las interacciones espaciales.

El objetivo central de esta tesis es contribuir a una mejor valuación de las medidas de bienestar, incluyendo, explícitamente, la dimensión espacial de los datos. Estas estimaciones tienen un impacto considerable en las decisiones de política como son la evaluación de proyectos, el diseño de impuestos y otros instrumentos de política relacionados con el medio ambiente. La precisión y la magnitud de las estimaciones pueden cambiar dependiendo de la especificación utilizada, así como también de la forma en que se incorporan los efectos espaciales. Por lo tanto, la obtención de estimaciones precisas de los beneficios asociados con las políticas públicas mejorará la calidad de las mismas y aumentará la eficacia de la política ambiental y fiscal. Además, proporcionará a la clase política una mayor cantidad de información sobre la sensibilidad de esas medidas y les permitirá tomar decisiones con mejor información.

La tesis está estructurada de la siguiente manera: El capítulo 1 presenta la metodología que permite incorporar al modelo de precios hedónicos los diferentes tipos de interacciones espaciales y la estrategia de estimación a utilizar. El capítulo 2 analiza el impacto de los diferentes niveles de riesgo de inundación sobre los precios de los inmuebles de la ciudad de La Plata. El capítulo 3 desarrolla un modelo que permite estimar la disposición marginal a pagar de los individuos por reducir la contaminación acústica en la ciudad de La Plata. El capítulo 4 presenta un modelo espacial por cuantil para la Ciudad Autónoma de Buenos Aires que permite analizar el hecho de que algunas características de los inmuebles pueden ser valoradas de manera distinta a lo largo de la distribución de los precios, teniendo en cuenta la existencia de las interacciones espaciales. La tesis finaliza con un apartado de consideraciones finales que resume los principales resultados de cada capítulo. 



\section{Modelos hedónicos espaciales}

\subsection{Introducción}

Los temas considerados en esta tesis poseen como características comunes la inexistencia de precios de mercado y la importancia de la localización geográfica. Ambos temas, por separado, han generado técnicas particulares como el análisis del modelo hedónico y los modelos de econometría espacial. En este capítulo se pretende mostrar lo conjunción de ambas técnicas que fundamentan el desarrollo de los demás capítulos.

Los riesgos de inundación, los cambios en la contaminación ambiental y las diversas características de los inmuebles son atributos que presentan dificultades en la asignación de un valor económico. En ausencia de un mercado explícito para cada uno de los atributos, la asignación de un valor debe realizarse por algún método alternativo. En la literatura existen al menos tres métodos alternativos para estimar estos valores económicos. Entre ellos se encuentra el enfoque de preferencias reveladas del que deriva el modelo de precios hedónicos que es el modelo utilizado a lo largo de los diferentes capítulos.

Desde la década de los sesenta, iniciando con el trabajo de Griliches (1961), se introduce el análisis hedónico para tratar la heterogeneidad de los bienes y para salvar el problema de la falta de mercado de algunos atributos. Los importantes aportes de Lancaster (1966) y, posteriormente, Rosen (1974) permiten que muchos estudios apliquen el modelo de precios hedónicos para conocer la relación entre los precios de los inmuebles respecto a las características constructivas y a los riesgos medioambientales, tales como inundaciones, terremotos, etc. Tal ha sido la flexibilidad del modelo hedónico que se ha convertido en la actualidad en una metodología habitual en temas de economía ambiental.

Por su parte, la localización geográfica es una característica inherente al mercado inmobiliario. Tradicionalmente, el problema de la ubicación geográfica ha sido considerado como una característica o atributo adicional dentro del enfoque de precios hedónicos. La estrategia más ampliamente adoptada es la construcción de diferentes medidas de distancia que permiten capturar la valoración de la posición espacial. De esta forma, la localización geográfica pasa a formar parte de una característica implícita del inmueble y le asigna un estatus similar a otros atributos, como la cantidad de dormitorios o el tamaño del inmueble. Este tipo de medición espacial ha posibilitado obtener estimaciones, por ejemplo, del impacto de la cercanía 
a plantas químicas, a accesos a carreteras, a lugares de recreación, etc. Sin embargo, los modelos considerados en esta tesis, además de considerar la dimensión espacial como una característica implícita del inmueble, consideran la posición geográfica de forma explícita dentro de la especificación del modelo econométrico. En este contexto, el término explícito indica que se reconoce que los datos poseen un ordenamiento espacial generalmente identificado por la latitud y por la longitud de cada observación ${ }^{1}$.

En econometría, la introducción de elementos espaciales explícitos ha dado lugar a una nueva y creciente subárea denominada econometría espacial. El surgimiento de esta rama econométrica puede ser explicado por, al menos, dos razones. Una de ellas ha sido el renovado interés por el rol del espacio y de las interacciones espaciales en la teoría económica reflejado en la Nueva Geografía Económica (Krugman, 1991; Fujita et al., 1999). Otra razón ha sido el desarrollo de diferentes técnicas computacionales tales como los sistemas de posicionamiento globales (comúnmente conocidos por sus siglas en inglés, GPS) y los sistemas de información geográfica (SIG) que facilitan la utilización de la información georeferenciada, así como de programas econométricos que incorporan la estimación espacial. Estos avances han posibilitado que diversas áreas de investigación como agricultura, medio ambiente, recursos naturales, tópicos de economía urbana y regional incorporen rápidamente elementos espaciales explícitos (Florax \& Van der Vlist, 2003).

A lo largo de los capítulos, la incorporación explícita de los elementos espaciales se realizará de forma pragmática, es decir, mediante la búsqueda de una especificación econométrica dirigida por los datos, data-driven approach. Otra vía alternativa, no aplicada aquí, es la modelización de los efectos espaciales desde la teoría económica conocida como theory-driven approach. La omisión de los efectos espaciales en un modelo econométrico puede generar diferentes inconvenientes. Los problemas menos severos incluyen potencial sesgo y pérdida de eficiencia en los coeficientes estimados, y, en el peor de los casos, se incluye la inconsistencia de los estimadores (Anselin, 1988). La detección y la incorporación de los efectos espaciales en los modelos hedónicos solucionan estos potenciales problemas y se han vuelto más habituales en los estudios empíricos del mercado inmobiliario, y dieron, así, lugar a los llamados modelos hedónicos espaciales.

A continuación se profundizará en el marco teórico y metodológico que sustenta los principales resultados de la presente tesis. En la segunda sección se presenta el modelo hedónico básico, entendido como la metodología tradicional que permite valorar los diferentes atributos y características que no poseen un mercado explícito. En esta sección, se discuten los principales supuestos y, también, las limitaciones del enfoque. La tercera sección presenta la discusión del modelo hedónico espacial; para ello, es necesario describir los diferentes efectos espaciales y las herramientas estadísticas que permiten la detección de dichos efectos. En la cuarta

\footnotetext{
${ }^{1}$ Formalmente, se asume que los datos observados son una realización de un proceso estocástico espacial. Para mayor detalle véase Arbia (2006, capítulo 2).
} 
sección se desarrolla el método de estimación de momentos generalizados y las correcciones por heterocedasticidad en el término de error. Finalmente, la quinta sección muestra la adecuada interpretación de los resultados según el modelo especificado.

\subsection{Modelo hedónico básico}

En la segunda mitad de los años sesenta, una nueva rama de la teoría de la utilidad fue desarrollada, donde la utilidad no se definía en función de los bienes, sino en función de las características que componían esos bienes. Con este desarrollo, Lancaster (1966) proporcionó la base microeconómica para el modelo hedónico centrado en el consumidor (Malpezzi, 2003) y no fue hasta Rosen (1974) que se desarrolló realmente un modelo de equilibrio de mercado que tuviese en cuenta tanto a los consumidores como a los productores. Rosen (1974) modeló como bienes diferentes lo que podría ser considerado esencialmente como un solo bien, lo que permitió que la utilidad de un individuo sea vista como una función de las características del bien y que los costos de producción dependan del tipo de bien.

La función de precios hedónicos define el equilibrio de mercado del bien objeto de estudio luego de que todas las interacciones entre la oferta y la demanda hayan tenido lugar. El modelo hedónico básico, aplicado al mercado inmobiliario, asume que la utilidad individual es una función que depende: de un bien compuesto $x$, de un vector $C$ de características del inmueble,de un vector $A$ de características ambientales, de un vector $S$ de características sociales y vecinales y, finalmente, de un vector $L$ de características de localización. De esta forma, las preferencias pueden representarse de la siguiente manera:

$$
U=u(x, C, A, S, L)
$$

Uno de los principales supuestos del modelo hedónico es que las preferencias son débilmente separables entre el inmueble y sus características. Específicamente, este supuesto implica que la demanda de inmuebles se puede escribir como una función del precio del inmueble, dejando de lado los precios de los otros bienes, representados en este modelo por el bien compuesto $x$. Por otra parte, el modelo hedónico supone que los consumidores son capaces de percibir todas las características del inmueble y que las mismas son tenidas en cuenta al momento de la adquisición del inmueble. Bajo estos supuestos, los individuos resuelven el siguiente problema al momento de comprar un inmueble:

$$
\text { máx } u\left(x, C_{i}, A_{i}, S_{i}, L_{i}\right), \text { s.a. } M=P_{i}-x,
$$

donde $M$ son los ingresos del hogar, $P_{i}$ es el precio del inmueble $i$. La condición de primer orden para cada característica define la disposición marginal a pagar ante cambios en los niveles de la misma. 
La función de precios hedónicos es una ecuación de precios de equilibrio en el que el precio del inmueble $i, P_{i}$, se define en función de las características del mismo:

$$
P_{i}=f\left(A_{i}, C_{i}, S_{i}, L_{i}\right)
$$

por lo que es posible estimar la disposición marginal a pagar del individuo por cualquier característica de la función de utilidad diferenciando la ecuación (1.2). La justificación del enfoque indica que si es posible estimar el precio hedónico de una determinada característica y la elección realizada por el consumidor, bajo los supuestos de optimización del consumidor, la estimación provee información local sobre las preferencias de los individuos o sobre la disposición a pagar por los atributos.

Para considerar que la ecuación (1.2) representa el equilibrio de mercado es necesario considerar cómo se determina la producción de cada uno de los atributos. Para ello, se debe asumir que existe un conjunto de productores de un bien heterogéneo, inmuebles en este caso, cuya función de costos depende de los diferentes atributos que componen el bien. La maximización de la función de beneficios requiere que el costo marginal de cada característica iguale a su precio hedónico. El equilibrio en este mercado de bienes heterogéneos requiere de una función de precios hedónicos tal como (1.2) que iguale la oferta y la demanda para cada atributo. Un análisis detallado sobre la formulación del modelo de equilibrio puede revisarse en Sheppard (1999).

Bajo estos supuestos, el análisis completo de un modelo hedónico se puede dividir en dos etapas. En primer lugar, la ecuación de precios hedónicos y, en segundo lugar, la función inversa de demanda para las características del inmueble.

La primera etapa se compone de dos pasos. El primer paso analiza el efecto de las diferentes características en los precios inmobiliarios y se obtienen mediante la estimación de la función de precios hedónicos. En dicha estimación es posible especificar adecuadamente la ecuación econométrica para luego estimar el precio marginal de cada característica del inmueble. Generalmente, esto se obtiene como la derivada parcial de la función de precios hedónicos con respecto a la variable considerada, aunque la expresión final de esta derivada dependerá de la forma del equilibrio de precios. El precio marginal de la característica analizada será lo que el mercado esté dispuesto a pagar por un cambio marginal en dicha característica.

La segunda etapa del modelo hedónico es la estimación de la función inversa de demanda, la cual consiste en calcular la disposición marginal a pagar (DMAP) obtenida en la etapa anterior evaluada en los niveles observados. La forma general de esta ecuación se puede escribir de la siguiente manera:

$$
D M A P_{i}=f\left(A_{i}, D_{i}\right)
$$


donde $i$ representa al individuo o al nivel más bajo de agregación disponible. Aquí, el precio marginal obtenido en la primera etapa está en el lado izquierdo de la ecuación, y la característica analizada $(A)$ y las otras curvas de la demanda $(D)$ se incluyen como variables explicativas.

Esta última etapa es útil para investigar el efecto de los cambios no marginales en las características analizadas. La función de precios hedónicos estimada en la primera etapa permite aislar el efecto de los cambios marginales en la característica analizada en los precios inmobiliarios. Si se dispone de mayor información a nivel individual sobre la disposición marginal a pagar, así como también de las características, es posible, hasta cierto punto, recuperar la demanda de las características inmobiliarias y, en particular, la demanda de las características que no tienen un mercado individual. Sin embargo, la falta de disponibilidad de datos ha limitado el número de aplicaciones que estiman la etapa final del modelo hedónico. Esta tesis no es la excepción y se centrará en la estimación de la disposición marginal a pagar, es decir, se concentra en la primera etapa del modelo hedónico. La falta de información disponible sobre las características individuales ha impedido la estimación de funciones de demanda que permitan estudiar la disposición marginal a pagar por cambios no marginales en el mercado inmobiliario.

Los modelos hedónicos tienen varias debilidades que han sido identificadas en la literatura y que deben tenerse en cuenta. En primer lugar, está el hecho de que la función de precios hedónicos muestra la relación entre el precio y las características asumiendo que el mercado se encuentra en equilibrio. Esta debilidad se debe a que el modelo de precios hedónicos asume que sólo existe un mercado y éste puede estar segmentado. Si este es el caso, los coeficientes estimados estarán sesgados y sería más apropiado estimar un modelo separado para cada segmento del mercado (Day, 2003). La presencia de los submercados en cualquier estudio hedónico contribuye no sólo a lograr la identificación de la función de demanda en la segunda etapa del modelo hedónico, sino, también, garantiza que si el mercado se encuentra segmentado, el equilibrio de cada segmento se describe por su propia función de precios hedónicos.

En segundo lugar, puede mencionarse el hecho de que el modelo de precios hedónicos supone que la función de utilidad es separable según las características del inmueble. Es posible que esto no siempre se cumpla y que sea necesario tener en cuenta las posibles interacciones de otros bienes con características inmobiliarias y sus efectos sobre la utilidad de los individuos. Por otra lado, este tipo de modelos supone que los cambios en los atributos pueden ser plenamente percibidos por las familias. Si algún cambio en un atributo no es percibido por los individuos, el efecto de ese cambio no será incorporado en las medidas de bienestar.

En tercer lugar, una crítica particular a algunas de las características analizadas a lo largo de esta tesis, como son la contaminación acústica y el riesgo hídrico, radica en que dado los supuestos del modelo hedónico el valor de este tipo de características sólo se capitalizará en los precios si los individuos son capaces de ver sus efectos y si realmente perciben los cambios en dichas características. Se ha planteado la duda sobre la capacidad de los individuos de percibir 
estas diferencias, con el argumento de que en la mayoría de los casos serían incapaces de hacerlo. Sin embargo, esto es puramente una cuestión empírica que debe ser testeada.

Por último, Freeman III (1979) señala que la existencia de una relación estadísticamente significativa entre el precio del inmueble y las demás características no implica una relación causal. Este punto es importante a la hora de interpretar los resultados. El hecho de que en muchos estudios - con diferentes conjuntos de datos - hayan encontrado una fuerte asociación entre estas características tiende a apoyar la hipótesis de que los cambios en diversas características son reflejados en los precios de los inmuebles; sin embargo, el análisis no es de tipo causal.

\subsection{Modelos hedónicos espaciales}

En esta sección el análisis se centrará en la incorporación de los efectos espaciales al modelo hedónico. El punto de partida es un modelo que cumple con todos los supuestos econométricos. En particular, el modelo hedónico básico considera que existe una variable dependiente, el precio del inmueble $(P)$, y un conjunto de variables explicativas $(X)$ tal que formalmente se lo puede especificar de la siguiente manera:

$$
P=X \beta+\mu,
$$

donde $P$ es la variable dependiente definida como un vector de dimensión $(N \times 1), X$ una matriz de variables explicativas, incluyendo una constante, de orden $(N \times K), \beta$ un vector de parámetros desconocidos de orden $(K \times 1)$ y $\mu$ un vector de error aleatorio de dimensión $(N \times 1)$. Entre los supuestos que cumple el modelo pueden mencionarse: forma funcional correcta, variables explicativas exógenas, no multicolinealidad, errores independiente e idénticamente distribuidos (i.i.d.) con media nula y varianza constante ${ }^{2}$.

En el mercado inmobiliario una afirmación ampliamente aceptada es que "la ubicación importa". Sin embargo, la inclusión explícita de dicha información en los modelos hedónicos no está extendida y su introducción presenta desafíos adicionales, como nuevos métodos de estimación y una interpretación alternativa de los resultados teóricos y empíricos.

Según Anselin (1988), surgen dos problemas cuando los datos tienen un componente de localización: (i) la dependencia espacial entre las observaciones, y (ii) la heterogeneidad espacial en las relaciones que se están especificando.

La econometría tradicional ha ignorado en gran medida estas dos cuestiones que violan habituales supuestos en los modelos de regresión. En cuanto a la dependencia espacial entre las observaciones, el modelo lineal general asume que el término de error es i.i.d. y

\footnotetext{
${ }^{2}$ La distribución normal del error puede o no ser requerida.
} 
la presencia dependencia espacial, ya sea en la parte sistemática (omisión de una variable explicativa endógena relevante) o en la parte aleatoria (correlación residual), viola este supuesto. La presencia de cualquiera de estos efectos espaciales genera que la estimación por mínimos cuadrados ordinarios (MCO) sea inapropiada dando lugar a la necesidad de enfoques alternativos de estimación.

Formalmente, la dependencia espacial para una variable relevante como los precios de inmueble significa que la observación $i$, identificada por su posición geográfica latitud-longitud $\left(\right.$ lat $_{i}, \operatorname{long}_{i}$ ), depende del comportamiento de la misma variable en otras observaciones $j$, vecinas a $i$ :

$$
P_{i}=f\left(P_{j}\right), i, j=1, \ldots, N, j \neq i \text {. }
$$

Hay dos razones por las cuales los datos observados en un punto del espacio pueden ser dependientes de los valores observados en otros lugares. En primer lugar, la recolección de datos asociados a unidades espaciales tales como códigos postales, partidos, provincias, fracciones censales, etc., podrían reflejar errores de medición. Esto podría ocurrir si los límites administrativos no reflejan con exactitud la naturaleza del proceso subyacente generador de datos - por ejemplo: las tasas de desempleo y la medición de la fuerza laboral - debido a que los trabajadores son móviles y pueden cruzar los límites administrativos para encontrar empleo en las zonas vecinas. Dichas tasas medidas según el lugar de residencia de los individuos podrían exhibir dependencia espacial. Una segunda razón de la existencia de dependencia espacial, y la más relevante para los trabajos realizados en esta tesis, radica en que la dimensión espacial de la actividad socio-demográfica, económica o regional que se está analizando pueda ser un aspecto importante en el objeto de estudio. La ciencia regional se basa en la premisa de que la ubicación y la distancia son fuerzas importantes en las actividades económicas, y en las nociones de interacción y de difusión efectos espaciales, en las jerarquías de lugar y en los efectos de derrame geográficos. La dependencia espacial que surge de las interacciones regionales subyacentes, a menudo observadas en datos regionales, sugiere la necesidad de cuantificar y de modelar la naturaleza de la forma funcional desconocida $f($.$) en la ecuación (1.4).$

En el mercado inmobiliario, la dependencia espacial puede surgir cuando los precios o las características de los inmuebles tienden a asumir valores similares en unidades geográficamente cercanas. Esta dependencia espacial puede darse cuando los precios o las características son similares entre los inmuebles cercanos; por ejemplo: el alto precio de una propiedad vendida empuja hacia arriba el precio de todos los inmuebles del vecindario, por lo que los precios de las propiedades vecinas actúan como una variable explicativa del precio ${ }^{3}$. La omisión de

\footnotetext{
${ }^{3}$ Esto podría darse si en el proceso de determinación de precios de venta de una propiedad los agentes inmobiliarios y los propietarios de viviendas como primer paso buscan precios de las propiedades vendidas recientemente en el barrio y luego fijan el precio de su propia casa en base a los precios encontrados. En este caso, es de esperar que el precio de venta de una propiedad sea mas elevado si en el vecindario el precio
} 
la dependencia espacial proveniente de la variable endógena genera que el término de error no cumpla con los supuestos habituales de Mínimos Cuadrados Ordinarios (MCO) tal que la estimación de los parámetros por este método será inconsistente.

Si la autocorrelación espacial proviene de un error de medición de las variables explicativas o por una omisión de una variable explicativa exógena, la estimación por MCO será ineficiente, además de que los estadísticos $t$ y $F$ estarán sesgados y la bondad del ajuste será engañosa (Anselin, 1992).

El segundo problema que plantea la localización se refiere a la heterogeneidad de la relación investigada sobre el espacio. Formalmente, esto implica que los parámetros de posición varíen en función de la ubicación ( lat $_{i}, \operatorname{long}_{i}$ ) de la observación $i$. En el caso más extremo se podría esperar una relación diferente en cada punto del espacio:

$$
P_{i}=X_{i} \beta_{i}+\epsilon_{i}
$$

donde los subíndices $i$ se relacionan con los puntos en el espacio (lat ${ }_{i}, \operatorname{long}_{i}$ ), $X_{i}$ representa una matriz de variables explicativas con un conjunto asociado de parámetros $\beta_{i}, P_{i}$ es la variable dependiente de la observación $i$ y $\epsilon_{i}$ denota una perturbación estocástica en la relación lineal. Una forma un poco más complicada de expresar esta idea es permitir que la función sea de tipo genérica, $f($.$) , tal que la ecuación (1.5) se reexprese como:$

$$
P_{i}=f_{i}\left(X_{i} \beta_{i}+\epsilon_{i}\right)
$$

La heterogeneidad espacial indica la presencia de diferencias sistemáticas en la ocurrencia de un fenómeno en las distintas zonas geográficas. La heterogeneidad espacial puede provenir de características de la demanda, de la oferta, de barreras institucionales, de discriminación racial, que pueden hacer que la distribución de los precios de la vivienda sea diferente a lo largo del territorio. Esta variación sistemática en el comportamiento de los individuos a través del espacio genera errores heterocedásticos y esto viola uno de los supuestos del modelo de regresión lineal clásica.

El detalle de las consecuencias de ignorar diferentes tipos de dependencia espacial ha sido ampliamente discutido en la literatura (ver Anselin, 1988; Anselin \& Bera, 1998; LeSage \& Pace, 2009). La incorporación de las diferentes alternativas de efectos espaciales al modelo (1.3) da lugar a un grupo de modelos denominados modelos hedónicos espaciales. A continuación se analizan las formas de detección de las interacciones espaciales.

de venta de inmuebles vendidos recientemente fue a un alto precio que el de una propiedad similar rodeado de casas vendidas recientemente a un precio bajo. Esto dará lugar a la dependencia espacial en los precios inmobiliarios. 


\subsubsection{Detección de efectos espaciales}

En base al modelo básico definido en la ecuación (1.3) se pueden establecer algunos estadísticos de dependencia espacial. La hipótesis nula de estos estadísticos se define por la ecuación (1.3), en la cual el término de error se asume que es independiente e idénticamente distribuido, pero claramente este no será el caso bajo la hipótesis alternativa que considera las interacciones espaciales.

El objetivo de estos estadísticos es encontrar evidencia que permita rechazar la hipótesis nula de no autocorrelación espacial. Ante la evidencia de componentes espaciales, se incorporarán diferentes efectos al modelo según la hipótesis alternativa especificada.

Antes de presentar los estadísticos espaciales es necesario definir la matriz de ponderadores espaciales. Esta matriz forma parte de uno de los temas operacionales cruciales en la econometría espacial, ya que requiere expresar formalmente la estructura espacial que va a ser incorporada al modelo. Es decir, debe determinarse cuales unidades en el sistema geográfico van a tener influencia sobre la unidad particular bajo consideración.

La matriz de ponderaciones espaciales o de pesos espaciales representa el patrón de vecindad entre distintas localizaciones (Anselin, 1992). Dado un conjunto de $N$ localizaciones $\left\{A_{1}, \ldots, A_{i}, \ldots, A_{N}\right\}$, se construye una matriz $W$ donde cada elemento de $W$ representa una medida de proximidad entre $A_{i}$ y $A_{j}, \forall i, j \in\left\{A_{1}, \ldots, A_{i}, \ldots, A_{N}\right\}$. La matriz $W$ será de orden $(N \times N)$ y se define formalmente como:

$$
W=\left[\begin{array}{cccccc}
0 & w_{1,2} & \cdots & w_{1, j} & \cdots & w_{1 N} \\
w_{2,1} & 0 & \cdots & w_{2, j} & \cdots & w_{2 N} \\
\vdots & \vdots & \ddots & \cdots & \cdots & \cdots \\
w_{i, 1} & w_{i, 2} & \vdots & 0 & \cdots & w_{i N} \\
\vdots & \vdots & \vdots & \vdots & 0 & \cdots \\
w_{N, 1} & w_{N, 2} & \vdots & w_{N, j} & \cdots & 0
\end{array}\right]
$$

donde las filas y las columnas corresponden a las observaciones de corte transversal, siendo $w_{i, j}$ los ponderadores o pesos espaciales. Estos pesos espaciales determinan la proximidad entre la localización $i$ y la localización $j$, para $i \neq j$. Por convención, ninguna localización puede ser vecina de si misma, lo que da por resultado que la diagonal principal de $W$ se encuentre formada por ceros.

La matriz de ponderaciones establece quién es vecino de quién y por ello debe establecerse un criterio que permita definir la vecindad de cada localización. No hay una regla general que permita elegir el criterio de vecindad siendo, por lo general, un a priori del investigador que sigue alguna hipótesis de interacción. Dentro de los criterios más habituales se encuentran los conceptos de contigüidad y de distancia, que utilizan la información geográfica y aseguran la 
exogeniedad de la matriz. Este punto es importante ya que se asume que $W$ contiene elementos exógenos respecto a la especificación econométrica elegida, por lo que en la ponderación de los vecinos no deben utilizarse variables consideradas explícitamente en el modelo analizado, ya sea la dependiente o las explicativas (Herrera, 2015) ${ }^{4}$. La importancia de este punto puede entenderse más cabalmente al momento de realizar el análisis de interpretación (sección 1.5).

El criterio contigüidad es utilizado cuando se analizan unidades administrativas irregulares considerando como vecino aquella unidad que comparte frontera. En el caso de observaciones puntuales, como los inmuebles, es habitual utilizar una función de distancia entre las unidades. En esta tesis será utilizado este último criterio considerando la función inversa de distancia, con un coeficiente de decaimiento igual a 2, el cual permite asignar a las propiedades inmobiliarias cercanas una mayor ponderación que las más lejanas de acuerdo con la siguiente formula:

$$
w_{i j}=\left(\frac{1}{d_{i j}}\right)^{2},
$$

donde $d_{i j}$ es la distancia entre el inmueble $i$ y el inmueble $j$.

Una vez elegido el criterio, la matriz de ponderaciones es estandarizada por fila para limitar la suma de los elementos de cada fila a la unidad. Teniendo en cuenta el mercado inmobiliario, significa conceptualmente que el precio de cada propiedad se verá afectado por el precio promedio ponderado de las propiedades cercanas y las propiedades más cercanas tendrán una mayor ponderación e impacto sobre la unidad de análisis.

Definida la matriz de ponderaciones, se presenta el primer estadístico espacial. Este estadístico es el test I de Moran (Moran, 1948), que utiliza los residuos MCO del modelo $(1.3)^{5}$ :

$$
I=\frac{N \widehat{\mu}^{\prime} W \widehat{\mu}}{S_{0} \widehat{\mu}^{\prime} \widehat{\mu}}
$$

donde $\widehat{\mu}$ es el vector de residuos de $\mathrm{MCO}, N$ es el número de observaciones y $S_{0}$ es la suma de todos los elementos de $W$. Bajo una matriz estandarizada por fila, el término $N / S_{0}=$ 1 puede ser ignorado. La hipótesis nula de test $I$ de Moran es no autocorrelación espacial. La distribución probabilística del estadístico es desconocida para muestras finitas, por lo que usualmente se utiliza una aproximación empírica por permutación. Otra opción es plantear la inferencia estadística bajo el supuesto de normalidad asintótica.

El problema con el test $I$ de Moran es que el rechazo de la hipótesis nula no brinda información sobre el posible modelo a especificar. La hipótesis alternativa es general y no da una guía clara sobre el tipo de estructura espacial que se encuentra en el proceso generador de datos.

\footnotetext{
${ }^{4} \mathrm{Al}$ respecto, Griffith (1996) establece un conjunto de lineamientos útiles para la elección de la matriz: (1) Es mejor utilizar una especificación razonable de la matriz de pesos geográficos que considerar todas las conexiones nulas; (2) modelos con bajos ordenes deben ser preferidos por sobre los modelos de altos ordenes; (3) en general, es mejor emplear una matriz de pesos subidenticada que una sobreidenticada.

${ }^{5}$ Otro estadístico similar al I de Moran es el test c de Geary (Geary, 1954).
} 
Como alternativa al estadístico $I$ de Moran, existe un conjunto de tests de Multiplicadores de Lagrange (LM) que resultan de la aplicación del principio de máxima verosimilitud. Estos estadísticos tienen la ventaja de que la hipótesis alternativa se encuentra bien definida o restringida.

Una primera hipótesis alternativa proviene de plantear la presencia de autocorrelación espacial en el término de error (estructura espacial residual). Esta estructura de dependencia puede deberse a un proceso autoregresivo o a uno de medias móviles. Sin pérdida de generalidad, se concentrará el análisis en la estructura autoregresiva dado que ante ambas alternativas el estadístico es el mismo (Burridge, 1980). Un modelo con errores autoregresivos espaciales asume que el término de error aleatorio del modelo (1.3), obedece a un proceso como:

$$
\mu=\lambda W \mu+\varepsilon
$$

donde $\lambda$ es el parámetro espacial autorregresivo, $W$ es una matriz de ponderadores espaciales no estocástica de orden $(N \times N)$ y $\varepsilon$ es un vector de innovaciones con distribución normal de media cero y varianza constante $\mathcal{N}\left(0, \sigma^{2} I\right)$.

El test LM para detectar autocorrelación espacial en el error (spatial error) establece la siguiente hipótesis nula y alternativa:

$H_{0}: \lambda=0$,

$H_{1}: \lambda \neq 0$,

siendo su fórmula:

$$
L M_{E R R O R}=\frac{1}{T_{1}}\left(\frac{\widehat{\mu}^{\prime} W \widehat{\mu}}{\widehat{\sigma}^{2}}\right) \sim \chi_{(1)}^{2},
$$

donde $T_{1}$ es igual a $\operatorname{tr}\left[\left(W^{\prime}+W\right) W\right]$ y $\widehat{\mu}$ los residuos de MCO y $\widehat{\sigma}^{2}=\widehat{\mu}^{\prime} \widehat{\mu} / N$.

Una segunda hipótesis alternativa proviene de plantear un modelo con estructura espacial sustantiva, modelo de rezago espacial (spatial lag). Este modelo incorpora un rezago espacial de la variable dependiente, $W P$, como variable explicativa. Formalmente puede expresarse de la siguiente manera:

$$
P=\rho W P+X \beta+\mu
$$

donde $\rho$ es un parámetro espacial autoregresivo, $\mu$ es un vector de errores de dimensión $(N \times 1)$ у $\mu \sim N\left(0, \sigma^{2} I\right)$.

El test para la detección de dependencia espacial sustantiva plantea como hipótesis nula y alternativa:

$H_{0}: \rho=0$, 
$H_{1}: \rho \neq 0$,

siendo su fórmula:

$$
L M_{L A G}=\frac{\left(\frac{\widehat{\mu}^{\prime} W \widehat{\mu}}{\widehat{\sigma}^{2}}\right)}{N \widehat{J}_{\rho \beta}} \sim \chi_{(1)}^{2}
$$

donde $\widehat{J}_{\rho \beta}$ es igual a $1 / N \widehat{\sigma}^{2}\left[(W X \widehat{\beta})^{\prime} M(W X \widehat{\beta})+T_{1} \widehat{\sigma}^{2}\right]$ con $M=I-X\left(X^{\prime} X\right)^{-1} X^{\prime}$.

Estos tests LM presentan como inconveniente la sensibilidad a diferentes tipos de errores de especificación. Por ejemplo, el $L M_{E R R O R}$ detecta autocorrelación espacial debido a la presencia de un rezago espacial de la variable endógena, $W P$, y lo mismo puede decirse del $L M_{L A G}$, que arroja falsos positivos cuando el término de error contiene un rezago espacial, $W \mu$. Debido a estas debilidades, Anselin et al. (1996) proponen dos nuevos multiplicadores de Lagrange diseñados para que su comportamiento sea robusto a dichos errores de especificación. El $L M_{E R R O R}^{*}$ analiza la falta de correlación en los residuos y es robusto a la omisión del término $W P$ :

$$
L M_{E R R O R}^{*}=\frac{\left[\left(\frac{\widehat{\mu}^{\prime} W \widehat{\mu}}{\widehat{\sigma}^{2}}\right)-T_{1}\left(N \widehat{J}_{\rho \beta}\right)^{-1}\left(\frac{\widehat{\mu}^{\prime} W P}{\widehat{\sigma}^{2}}\right)\right]^{2}}{\left[T_{1}-T_{2}\left(N \widehat{J}_{\rho \beta}\right)^{-1}\right]} \sim \chi_{(1)}^{2}
$$

Por su parte, el $L M_{L A G}^{*}$ permite detectar la autocorrelación espacial sustantiva, que es robusta a la presencia de estructura espacial en el término de error:

$$
L M_{L A G}^{*}=\frac{\left[\left(\frac{\widehat{\mu}^{\prime} W P}{\widehat{\sigma}^{2}}\right)-\left(\frac{\widehat{\mu}^{\prime} W \widehat{\mu}}{\widehat{\sigma}^{2}}\right)\right]^{2}}{N \widehat{J}_{\rho \beta}-T_{1}} \sim \chi_{(1)}^{2}
$$

Para mayor detalle sobre la derivación de estos tests se puede consultar Anselin et al. (1996).

\subsubsection{Especificación de modelos hedónicos espaciales}

El uso combinado del test I de Moran y los tests LM permiten incorporar elementos espaciales de acuerdo con el rechazo o no de cada una de las hipótesis nulas. Tal es así que puede establecerse una estrategia de especificación de los diferentes modelos hedónicos espaciales:

- Si $I$ de Moran rechaza $H_{0}$, entonces existe evidencia a favor de la inclusión de elementos espaciales.

- Si solo los tests $L M_{E R R O R}$, y $L M_{E R R O R}^{*}$ rechazan $H_{0}$, hay evidencia a favor de un modelo de error espacial (SEM, spatial error model): 


$$
\begin{aligned}
P & =X \beta+\mu, \\
\mu & =\lambda W \mu+\varepsilon .
\end{aligned}
$$

Este modelo corregirá los errores por heterogeneidad espacial y la interpretación de los resultados de estimación será similar a la que se obtiene en el modelo MCO. El ajuste espacial solo afectará a los errores estándares.

- Si solo los tests $L M_{L A G}$, y $L M_{L A G}^{*}$ rechazan $H_{0}$, hay evidencia a favor de un modelo de rezago espacial (SLM, spatial lag model).

$$
P=\rho W P+X \beta+\mu \text {. }
$$

Este modelo genera una dinámica espacial no presente en el modelo MCO. La presencia de una variable endógena espacial como explicativa genera que la interpretación habitual de los resultados deba modificarse. En la sección 1.5 se detallan las implicancias del modelo.

- Si ambos tests robustos, $L M_{L A G}^{*}$ y $L M_{E R R O R}^{*}$, rechazan $H_{0}$, entonces se deberán incorporar elementos espaciales en la parte sistemática y aleatoria (SARAR).

$$
\begin{aligned}
P & =\rho W P+X \beta+\mu, \\
\mu & =\lambda W \mu+\varepsilon .
\end{aligned}
$$

Este modelo es una combinación de los modelos SLM y SEM, por lo que los errores estándares serán corregidos por heterogeneidad espacial y el modelo tendrá una dinámica espacial que requiere una adecuación de la interpretación de los efectos marginales de las variables explicativas. La sección 1.5 presenta en detalle esta adecuación.

Si no se rechaza $H_{0}$ bajo ninguno de los tests, el modelo adecuado será un modelo hedónico básico, sin componentes espaciales.

\subsection{Estimación de los modelos hedónicos espaciales}

Las alternativas de estimación de los modelos espaciales han sido tradicionalmente de dos tipos. La primera es por máxima verosimilitud (ML por sus siglas en inglés), que asume una distribución normal de la perturbación aleatoria. La segunda utiliza variables instrumentales o su versión generalizada, conocida como el Método de Momentos Generalizado (GMM por sus siglas en inglés), que recurre a la teoría asintótica sin necesidad del supuesto de normalidad. 
La principal diferencia entre estas alternativas radica en su funcionamiento bajo los supuestos de las innovaciones $\varepsilon$, particularmente el supuesto de homocedasticidad o de heterocedasticidad. La ventaja del estimador de Método de Momentos Generalizado es que produce estimaciones consistentes en cualquiera de los dos $\operatorname{casos}^{6}$. El estimador ML produce estimaciones consistentes en el caso i.i.d., pero en general no en el caso heterocedástico. Puede consultarse Lee (2003) para algunos resultados formales para el estimador ML y Arraiz et al. (2010) para pruebas de que el estimador ML no suele producir estimaciones consistentes en el caso heterocedástico.

En esta tesis se utilizará el método GMM, ya que tiene la ventaja de que utiliza la teoría asintótica sin necesidad del supuesto de normalidad de las perturbaciones y produce estimaciones consistentes en los casos en los que las innovaciones se distribuyan de manera heterocedástica. Para esto, se utilizará el estimador heteroskedasticity autocorrelation consistent (HAC) desarrollado por Kelejian \& Prucha (2007).

Los principales precursores de este método son los trabajos de Kelejian \& Prucha (1998, 1999), con generalizaciones recientes realizadas por Kelejian \& Prucha (2010), Arraiz et al. (2010) y Drukker et al. (2013).

Como punto de partida se considerará el modelo hedónico espacial más complejo, el SARAR, formalmente:

$$
\begin{aligned}
P & =\rho W P+X \beta+\mu, \\
\mu & =\lambda W \mu+\varepsilon
\end{aligned}
$$

donde $\rho$ y $\lambda$ son parámetros espaciales autoregresivos, $\mu$ es un vector de errores de dimensión $(N \times 1)$ y $\varepsilon \sim\left(0, \sigma^{2} I\right)$; las innovaciones $\varepsilon$ pueden modificarse para la inclusión del caso heterocedástico.

\subsubsection{Modelo de rezago espacial (SLM)}

El modelo de rezago espacial puede verse como un modelo restringido del SARAR. Un SLM es un modelo producto de las ecuaciones (1.13) y (1.14), bajo la condición de $\lambda=0$, que puede expresarse de la siguiente manera:

$$
P=Z \delta+\mu
$$

donde $Z=[X, W P]$ y $\delta^{\prime}=\left[\beta^{\prime}, \rho\right]$ con $\mu \sim N\left(0, \sigma^{2} I\right)$ siendo $I$ una matriz identidad de orden $(N \times N)$.

\footnotetext{
${ }^{6}$ Para los resultados formales y los debates respecto a ambos procedimientos véase Kelejian \& Prucha (1998, 1999); Arraiz et al. (2010); Drukker et al. (2013).
} 
La estimación de este modelo se basa en el enfoque de variables instrumentales (IV). La idea básica es simple, el modelo (1.15) no puede estimarse por los métodos habituales debido a que existe una variable endógena, $W P$, que está correlacionada con el término de error. La alternativa es encontrar un instrumento, $H$, fuertemente correlacionado con la variable que genera endogeneidad, $W P$, pero que no se correlacione asintóticamente con el error. Es decir, el instrumento $H$ debe cumplir con:

$\operatorname{plim}\left(\begin{array}{l}1 \\ n\end{array}\right) H^{\prime} W P=M_{H W P}$,

y

$\operatorname{plim}\left(\begin{array}{l}1 \\ n\end{array}\right) H^{\prime} \mu=0$

siendo $M_{H W P}$ una matriz finita no singular.

La forma reducida del SLM puede definirse como: $E(P)=(I-\rho W)^{-1}(X \beta+u)$ donde $(I-\rho W)^{-1}$ puede expresarse como una progresión: $(I-\rho W)^{-1}=I+\rho W+\rho^{2} W+\rho^{3} W+\ldots$ Entonces, el valor esperado de $P$ puede expresarse como: $E(P)=X \beta+\rho W X \beta+\rho^{2} W X \beta+\rho^{3} W X \beta+\ldots$, y $E(W P)=W X \beta+\rho W^{2} X \beta+\rho^{2} W^{2} X \beta+\rho^{3} W^{2} X \beta+\ldots \ldots$

Luego, $E(W P)$ se relaciona linealmente con $W X, W^{2} X, W^{3} X, \ldots, W^{q} X, \ldots$, siendo instrumentos directos de la variable endógena $W P$. Diferentes experimentos Monte Carlo muestran un buen comportamiento para $q=2$, por lo que habitualmente se utilizan $W X$ y $W^{2} X$ como el número máximo de instrumentos. El uso de órdenes mayores tiende a generar problemas de multicolinealidad entre los regresores. Aplicando esta idea al modelo (1.15) se obtiene el estimador de variables instrumentales (IV):

$\hat{\delta}=\left(\widehat{Z}^{\prime} Z\right)^{-1} \widehat{Z}^{\prime} P$

donde $\widehat{Z}=Q_{H} Z=[X, \widehat{W P}], \widehat{W P}=Q_{H} W P$ y $Q_{H}=H\left(H^{\prime} H\right)^{-1} H^{\prime}$, siendo los instrumentos elegidos $H=[X, W X]$.

Kelejian \& Prucha (1998) demuestran que:

$$
N^{1 / 2}(\widehat{\delta}-\delta) \stackrel{d}{\rightarrow} \mathcal{N}\left(\begin{array}{c}
0, \sigma^{2} \underset{N \rightarrow \infty}{N \rightarrow \infty} \\
\end{array} \quad N\left[\widehat{Z}^{\prime} \widehat{Z}\right]^{-1}\right)
$$

La estimación IV de estos parámetros puede ser obtenida como un estimador de mínimos cuadrados en dos etapas (2SLS). A continuación se describen los pasos necesarios para realizar dicha estimación:

1. Estimar la relación entre los instrumentos $H=[X, W X]$ y $Z=[X, W P]$, donde $Z=H \theta+\varepsilon$, y $\theta=\left(H^{\prime} H\right)^{-1} H^{\prime} Z$, tal que los valores predichos del modelo son: $\widehat{Z}=H \widehat{\theta}=H\left(H^{\prime} H\right)^{-1} H^{\prime} Z=$ $Q_{H} Z$.

2. Estimar $P=Z \delta+\varepsilon$, usando los resultados del paso anterior:

$\hat{\delta}=\left(\widehat{Z}^{\prime} Z\right)^{-1} \widehat{Z}^{\prime} P$, 
donde $\widehat{Z}=Q_{H} Z=[X, \widehat{W P}], \widehat{W P}=Q_{H} W P$.

Si se asume que el término de error es independiente pero heterocedástico, entonces se modifica la matriz de varianzas-covarianzas asintótica de $\widehat{\delta}$, y se adquiere la forma sándwich: $\left(\widehat{Z}^{\prime} \widehat{Z}\right)^{-1} \widehat{Z}^{\prime} \widehat{\Sigma} \widehat{Z}\left(\widehat{Z}^{\prime} \widehat{Z}\right)^{-1}$ donde $\widehat{\Sigma}$ es una matriz diagonal cuyos elementos $i$-ésimos son $\widetilde{\mu}_{i}^{2}$, con $\widetilde{\mu}_{i}=P_{i}-Z_{i} \widehat{\delta}$.

\subsubsection{Modelo de error espacial (SEM)}

El modelo de error espacial puede expresarse como:

$$
P=Z \delta+\mu, \text { con } \mu=\lambda W \mu+\varepsilon
$$

donde $Z=X, \delta=\beta, \rho$ es un parámetro autoregresivo espacial, siendo las innovaciones $\varepsilon \sim\left(0, \sigma^{2} I\right)$, e $I$ una matriz identidad de orden $(N \times N)$.

El objetivo es obtener un estimador consistente de $\lambda$. El primer paso consiste en generar un estimador inicial para $\lambda$ que surge de las condiciones de momentos cuadráticos de las innovaciones:

$E\left(\varepsilon^{\prime} A_{s} \varepsilon\right)=0, s=1, \ldots, S$,

donde las matrices $A_{s}$ satisfacen $\operatorname{tr}\left(A_{s}\right)=0$.

El estimador inicial GMM viene dado por:

$$
\widetilde{\lambda}=\operatorname{argmin}\left\{\left[\tilde{\Gamma}\left(\frac{\lambda}{\lambda^{2}}\right)-\tilde{\omega}\right]^{\prime}\left[\tilde{\Gamma}\left(\frac{\lambda}{\lambda^{2}}\right)-\tilde{\omega}\right]\right\}
$$

donde

$$
\widetilde{\Gamma}=\frac{1}{n}\left[\begin{array}{cc}
\widehat{\mu}\left(A_{1}+A_{1}^{\prime}\right) \widehat{\bar{\mu}} & -\widehat{\widehat{\mu}}^{\prime} A_{1} \widehat{\bar{\mu}} \\
\vdots & \vdots \\
\widehat{\mu}\left(A_{S}+A_{S}^{\prime}\right) \widehat{\bar{\mu}} & -\widehat{\bar{\mu}}^{\prime} A_{S} \widehat{\bar{\mu}}
\end{array}\right], \tilde{\omega}=\frac{1}{n}\left[\begin{array}{c}
\widehat{\mu}^{\prime} A_{1} \widehat{\mu} \\
\vdots \\
\widehat{\mu}^{\prime} A_{S} \widehat{\mu}
\end{array}\right],
$$

siendo $\widehat{\mu}=P-Z^{\prime} \widehat{\beta}$ y $\widehat{\bar{\mu}}=W \widehat{\mu}$.

Así planteado, el estimador GMM proviene de un estimador de mínimos cuadrados no-lineales. Generalmente, se utilizan dos ecuaciones $(\mathrm{S}=2)$.

Bajo homocedasticidad de las innovaciones: 


$$
A_{1}=\left[1+\left\{\frac{1}{n} \operatorname{tr}\left(W^{\prime} W\right)\right\}^{2}\right]^{-1}\left\{W^{\prime} W-\frac{1}{n} \operatorname{tr}\left(W^{\prime} W\right) I_{n}\right\}
$$

y

$$
A_{2}=W
$$

Una vez obtenida la estimación inicial de $\lambda$, se procede a obtener los coeficientes del modelo (1.16). Para ello, se utiliza la transformación Cochrane-Orcutt:

$$
\begin{gathered}
(I-\lambda W) P=(I-\lambda W) Z \beta+\varepsilon, \\
P_{\lambda}=Z_{\lambda} \beta+\varepsilon .
\end{gathered}
$$

Bajo esta especificación, se utiliza la estimación GMM para obtener:

$\widetilde{\lambda}=\left(\widehat{Z}_{\widetilde{\lambda}}^{\prime} \widehat{Z}_{\widetilde{\lambda}}\right)^{-1} \widehat{Z}_{\widetilde{\lambda}}^{\prime} P_{\widetilde{\lambda}}$

donde $P_{\widetilde{\lambda}}=(I-\widetilde{\lambda} W) P, Z_{\widetilde{\lambda}}=(I-\widetilde{\lambda} W) Z, \widehat{Z}_{\widetilde{\lambda}}=Q_{H} Z_{\widetilde{\lambda}}=X\left(X^{\prime} X\right)^{-1} X^{\prime} Z_{\widetilde{\lambda}}$.

El procedimiento completo es conocido como mínimos cuadrados espaciales en dos etapas generalizadas (GS2SLS, por sus siglas en ingles). Posterior a la estimación de los parámetros del modelo, puede darse un paso adicional para obtener un estimador eficiente de $\lambda$. Para ello, se utilizan los residuos del procedimiento GS2SLS:

$$
\widetilde{\lambda}=\arg \min \left\{\left[\tilde{\Gamma}\left(\frac{\lambda}{\lambda^{2}}\right)-\tilde{\omega}\right]^{\prime}\left\{\widehat{\Psi}_{\vec{\lambda}}^{\lambda \lambda}\right\}^{-1}\left[\tilde{\Gamma}\left(\frac{\lambda}{\lambda^{2}}\right)-\tilde{\omega}\right]\right\},
$$

donde $\widehat{\Psi}_{\widetilde{\lambda}}^{\lambda \lambda}$ es un estimador de la matriz de varianzas-covarianzas del vector de momentos muestrales (normalizados) basados en los residuos GS2SLS. Bajo homocedasticidad, los elementos $r, s(\operatorname{con} r, s=1,2)$ de $\widehat{\Psi}_{\widetilde{\lambda}}^{\lambda \lambda}$ están dados por:

$$
\begin{aligned}
\widehat{\Psi}_{\widetilde{\lambda}}^{\lambda \lambda}= & \left\{\widetilde{\sigma}_{\widetilde{\lambda}}^{2}\right\}^{2} \frac{1}{2 n} \operatorname{tr}\left\{\left(A_{r}+A_{r}^{\prime}\right)\left(A_{s}+A_{s}^{\prime}\right)\right\}+\frac{1}{n} \widetilde{\sigma}_{\widetilde{\lambda}}^{2} \widetilde{a}_{\tilde{\lambda} r}^{\prime} \widetilde{a}_{\tilde{\lambda} s} \\
& +\frac{1}{n}\left[\widetilde{\xi}_{\widetilde{\lambda}}^{(4)}-3\left\{\widetilde{\sigma}_{\widetilde{\lambda}}^{2}\right\}^{2}\right] \operatorname{vec}_{D}\left(A_{r}\right)^{\prime} \operatorname{vec}_{D}\left(A_{s}\right) \\
& +\frac{1}{n} \widetilde{\xi}_{\widetilde{\lambda}}^{(3)}\left\{\widetilde{a}_{\tilde{\lambda} r}^{\prime} \operatorname{vec}_{D}\left(A_{s}\right)+\widetilde{a}_{\tilde{\lambda} s}^{\prime} \operatorname{vec}_{D}\left(A_{r}\right)\right\}
\end{aligned}
$$

siendo

$\widetilde{\mathrm{a}}_{\widetilde{\lambda} r}=\widehat{\mathrm{T}}_{\widetilde{\lambda}} \widetilde{\alpha}_{\widetilde{\lambda}_{r}}$,

$\widehat{\mathrm{T}}_{\widetilde{\lambda}}=\mathrm{H} \widehat{Q}_{\widetilde{\lambda}}$, 
$\widehat{Q}_{\widetilde{\lambda}}=\widehat{V}_{H H}^{-1} \widehat{V}_{\widetilde{\lambda} H Z}\left\{\widehat{V}_{\widetilde{\lambda} H Z}^{\prime} \widehat{V}_{H H}^{-1} \widehat{V}_{\widetilde{\lambda}_{H Z}^{\prime}}^{\prime}\right\}^{-1}$,

$\widehat{V}_{H H}=\left(\frac{1}{n} H^{\prime} H\right)$,

$\widehat{V}_{\widetilde{\lambda} H Z}=\left(\frac{1}{n} H^{\prime} Z_{\widetilde{\lambda}}\right)$,

$Z_{\widetilde{\lambda}}=(I-\tilde{\lambda} W) Z$,

$\widetilde{\mathrm{a}}_{\widetilde{\lambda} r}=-\frac{1}{n}\left\{Z_{\widetilde{\lambda}}^{\prime}\left(A_{r}+A_{r}^{\prime}\right) \widehat{\varepsilon}_{\widetilde{\lambda}}\right\}$,

$\widehat{\varepsilon}_{\widetilde{\lambda}}=(I-\tilde{\lambda} W) \widehat{\mu}$,

$\widehat{\sigma}_{\widetilde{\lambda}}^{2}=\frac{1}{n} \widehat{\varepsilon}_{\widetilde{\lambda}}^{\prime} \widehat{\varepsilon}_{\widetilde{\lambda}}$,

$\widehat{\xi}_{\widetilde{\lambda}}^{(3)}=\frac{1}{n} \sum_{i=1}^{n} \widehat{\varepsilon}_{i \widetilde{\lambda}}^{3}$,

$\widehat{\xi}_{\widetilde{\lambda}}^{(4)}=\frac{1}{n} \sum_{i=1}^{n} \widehat{\varepsilon}_{i \widetilde{\lambda}}^{4}$

La estimación obtenida asume innovaciones homocedásticas. En el caso de heterocedasticidad, se debe modificar en el estimador inicial el elemento $A_{1}: A_{1}=W^{\prime} W-\operatorname{diag}\left(W^{\prime} W\right)$.

En la segunda etapa, que realiza una estimación eficiente de $\lambda$ usando los residuos GS2SLS, se modifica el elemento $r, s$ de $\widehat{\Psi}_{\tilde{\lambda}}^{\lambda \lambda}$ :

$$
\widehat{\Psi}_{\widetilde{\lambda}}^{\lambda \lambda}=\frac{1}{2 n} \operatorname{tr}\left\{\left(A_{r}+A_{r}^{\prime}\right) \widehat{\Sigma}_{\widetilde{\lambda}}\left(A_{s}+A_{s}^{\prime}\right)\right\}+\frac{1}{n} \widetilde{\sigma}_{\widetilde{\lambda}}^{2} \widetilde{\widetilde{a}}_{\tilde{\lambda} r}^{\prime} \widehat{\Sigma}_{\widetilde{\lambda}} \widetilde{\mathrm{a}}_{\tilde{\lambda} s}
$$

donde $\widehat{\Sigma}_{\widetilde{\lambda}}$ es una matriz diagonal cuyo elemento $i$-ésimo es $\widehat{\varepsilon}_{\widetilde{i} \widetilde{\lambda}}^{2}$ y $\widehat{\varepsilon}_{\widetilde{\lambda}}$ y $\widetilde{a}_{\widetilde{\lambda} r}$ son definidos como en el caso homocedástico.

\subsubsection{Modelo SARAR}

El modelo SARAR puede expresarse de la siguiente manera:

$P=Z \delta+\mu$ y $\mu=\lambda W \mu+\varepsilon$, donde $Z=[X, W P]$ y $\delta^{\prime}=\left[\beta^{\prime}, \rho\right]$ con $\varepsilon \sim\left(0, \sigma^{2} I\right)$.

El procedimiento de estimación del modelo SARAR es una combinación de los pasos dados en los modelos anteriores y puede ser resumido en los cuatro pasos siguientes:

Paso 1: Estimar por mínimos cuadrados en dos etapas (2SLS) la ecuación $P=Z \delta+\mu$, y así obtener:

$\widehat{\delta}=\left(\widehat{Z}^{\prime} \widehat{Z}\right)^{-1} \widehat{Z}^{\prime} P$

donde $\widehat{Z}=Q_{H} Z=[X, \widehat{W P}], \widehat{W P}=Q_{H} W P$ y $Q_{H}=H\left(H^{\prime} H\right)^{-1} H^{\prime}$ siendo los instrumentos elegidos $H=[X, W X]$.

Paso 2: Obtener $\widehat{\mu}=P-Z \widehat{\delta}, \overline{\widehat{\mu}}=W \widehat{\mu}$. Usando estos vectores residuales obtener el estimador inicial GMM minimizando: 
$\widetilde{\lambda}=\arg \min \left\{\left[\widetilde{\Gamma}\left(\frac{\lambda}{\lambda^{2}}\right)-\widetilde{\omega}\right]^{\prime}\left[\widetilde{\Gamma}\left(\frac{\lambda}{\lambda^{2}}\right)-\widetilde{\omega}\right]\right\}$.

Paso 3: Estimar $\widehat{\delta}$ por GS2SLS. Se utiliza la estimación inicial GMM, $\widehat{\lambda}$ en la transformación Cochrane-Orcutt para obtener

$\widehat{\delta}=\left(\widehat{Z}_{\tilde{\lambda}}^{\prime} \widehat{Z}_{\tilde{\lambda}}\right)^{-1} \widehat{Z}_{\tilde{\lambda}}^{\prime} P_{\tilde{\lambda}}$

donde $P_{\tilde{\lambda}}=(I-\tilde{\lambda} W) y, Z_{\tilde{\lambda}}=(I-\tilde{\lambda} W) Z, \widehat{Z}_{\tilde{\lambda}}=P_{H} \widehat{Z}_{\tilde{\lambda}}=H\left(H^{\prime} H\right)^{-1} H^{\prime} Z_{\tilde{\lambda}}$

Paso 4: Obtener el estimador eficiente y consistente de GMM. Utilizando los residuos del procedimiento GS2SLS se estima:

$\widetilde{\lambda}=\arg \min \left\{\left[\widetilde{\Gamma}\left(\begin{array}{c}\lambda \\ \lambda^{2}\end{array}\right)-\widetilde{\omega}\right]^{\prime}\left\{\widehat{\Psi}_{\widetilde{\lambda}}^{\lambda \lambda}\right\}^{-1}\left[\widetilde{\Gamma}\left(\begin{array}{c}\lambda \\ \lambda^{2}\end{array}\right)-\widetilde{\omega}\right]\right\}$,

Luego de haber computado los estimadores $\widehat{\delta}=(\widehat{\beta}, \widehat{\rho})$ y $\widetilde{\lambda}$, el próximo paso es computar un estimador consistente de la matriz de varianzas-covarianzas, $\Upsilon$. El estimador viene dado por $n \widehat{\Upsilon}$ donde:

$$
\widehat{\Upsilon}=\left(\begin{array}{cc}
\widehat{\Upsilon}^{\delta \delta} & \widehat{\Upsilon}^{\delta \lambda} \\
\widehat{\Upsilon}^{\delta \lambda^{\prime}} & \widehat{\Upsilon}^{\lambda \lambda}
\end{array}\right)
$$

con

$\widehat{\Upsilon}^{\delta \delta}=\widehat{Q}_{\widetilde{\lambda}}^{\prime} \widehat{\Psi}_{\widetilde{\lambda}}^{\delta \delta} \widehat{Q}_{\widetilde{\lambda}}$

$\widehat{\Upsilon}^{\delta \lambda}=\widehat{Q}_{\widetilde{\lambda}}^{\prime} \widehat{\Psi}_{\tilde{\lambda}}^{\delta \lambda} \widehat{Q}_{\widetilde{\lambda}}\left\{\widehat{\Psi}_{\widetilde{\lambda}}^{\lambda \lambda}\right\}^{-1} \widehat{J}\left[\widehat{J}^{\prime}\left\{\widehat{\Psi}_{\widetilde{\lambda}}^{\lambda \lambda}\right\}^{-1} \widehat{J}\right]^{-1}$,

$\widehat{\Upsilon}^{\lambda \lambda}=\widehat{J}\left[\widehat{J}^{\prime}\left\{\widehat{\Psi}_{\widetilde{\lambda}}^{\lambda \lambda}\right\}^{-1} \widehat{J}\right]^{-1}$

$\widehat{J}=\widehat{\Gamma}\left(\begin{array}{c}1 \\ 2 \widetilde{\lambda}\end{array}\right)$

Bajo el supuesto de homocedasticidad de las innovaciones, se utilizan:

$\widehat{\Psi}_{\widetilde{\lambda}}^{\delta \delta}=\widehat{\sigma}_{\widetilde{\lambda}}^{2} \widehat{V}_{H H}$,

$\widehat{\Psi}_{\widetilde{\lambda}}^{\delta \lambda}=\frac{1}{n} \widehat{\sigma}_{\widetilde{\lambda}}^{2} H^{\prime}\left\{\mathrm{a}_{1 \widetilde{\lambda}}, \mathrm{a}_{2 \widetilde{\lambda}}\right\}+\frac{1}{n} \xi_{\widetilde{\lambda}}^{3} H^{\prime}\left\{\operatorname{vec}_{D}\left(A_{1}\right), \operatorname{vec}_{D}\left(A_{2}\right)\right\}$

Cuando se especifica heterocedasticidad, la estructura de los siguientes elementos se modifica (además de las modificaciones presentadas en los pasos previos):

$\widehat{\Psi}_{\widetilde{\lambda}}^{\delta \delta}=\frac{1}{n} H^{\prime} \widehat{\Sigma}_{\widetilde{\lambda}} H$

$\widehat{\Psi}_{\widetilde{\lambda}}^{\delta \lambda}=\frac{1}{n} H^{\prime} \widehat{\Sigma}_{\widetilde{\lambda}}\left\{\mathrm{a}_{1 \widetilde{\lambda}}, \mathrm{a}_{2 \widetilde{\lambda}}\right\}$ 


\subsection{Interpretación de los resultados}

Una vez realizada la estimación es importante interpretar adecuadamente los resultados obtenidos. El modelo SEM no conlleva mayor dificultad que la que puede presentar un modelo por MCO, la única corrección espacial de este modelo radica en los errores estándares.

Para el caso de los modelos SLM y SARAR, es necesario realizar un esfuerzo adicional. En particular, el modelo SLM fue ampliamente utilizado en los pioneros trabajos de econometría espacial ya que la inclusión de un rezago espacial endógeno permitía obtener estimaciones de los llamados efectos contagio, derrame o desbordamiento, dependiendo del tópico analizado. La interpretación se centraba en la significancia y el signo del parámetro estimado de $\rho$ como evidencia de la importancia de los efectos espaciales. Sin embargo, LeSage \& Pace (2009) destacaron la parcial, y muchas veces errónea, interpretación de estos resultados y mostraron la necesidad de profundizar esta interpretación .

El punto inicial que plantean LeSage \& Pace (2009) es que para obtener los efectos derrame debe considerarse las derivadas parciales de una determinada variable explicativa sobre la variable dependiente. En concreto, si se asume que se estimará un modelo como $P=\rho W P+X \beta+\mu$, la obtención de los efectos marginales deberá considerar la forma reducida del modelo:

$$
P=(I-\rho W)^{-1}(X \beta+\mu)
$$

tal que aplicando el operador de esperanza se obtiene:

$$
E(P)=(I-\rho W)^{-1} X \beta
$$

siendo esta última expresión la que se utilizará para derivar los efectos marginales totales. Obsérvese que el operador de esperanza asume que los elementos contenidos en $W$ son exógenos, tales como las variables explicativas, y solo afecta al término de error. Este es uno de los puntos por los que las ponderaciones espaciales en $W$ no deben relacionarse con la variable dependiente.

Ahora, la matriz de derivadas parciales del valor esperado de $P$ respecto al cambio en una unidad de la $k$-ésima variable explicativa en $X$ puede ser representada como: 


$$
\begin{aligned}
{\left[\begin{array}{lll}
\frac{\partial E(P)}{\partial X_{1 k}} & \cdots & \frac{\partial E(P)}{\partial X_{N k}}
\end{array}\right] } & =\left[\begin{array}{ccc}
\frac{\partial E\left(P_{1}\right)}{\partial X_{1 k}} & \cdots & \frac{\partial E\left(P_{1}\right)}{\partial X_{N k}} \\
\vdots & \ddots & \vdots \\
\frac{\partial E\left(P_{N}\right)}{\partial X_{1 k}} & \cdots & \frac{\partial E\left(P_{N}\right)}{\partial X_{N k}}
\end{array}\right], \\
& =(I-\rho W)^{-1}\left[\begin{array}{cccc}
\beta_{k} & 0 & \cdots & 0 \\
0 & \beta_{k} & \cdots & 0 \\
\vdots & \vdots & \ddots & \vdots \\
0 & 0 & \cdots & \beta_{k}
\end{array}\right], \\
& =S\left[\beta_{k} I\right],
\end{aligned}
$$

donde $w_{i j}$ es el elemento $(i, j)$ - ésimo de $W, \beta_{k}$ es el $k$-ésimo elemento del vector $\beta$ y $S$ es igual a $(I-\rho W)^{-1}$. Nuevamente, se considera que los elementos de $W$ no se ven afectados por por cambios marginales en la variable explicativa $k$-ésima. Si la matriz $W$ fue construida con información relacionada con alguna variable explicativa, la presente interpretación no sería válida.

En la expresión (1.17) puede observarse la presencia de un efecto directo y otro indirecto. El efecto directo es representado por los elementos de la diagonal principal de la ecuación (1.17). Este impacto será diferente para cada región, por lo que no existe un único efecto directo. Por su parte, el efecto indirecto (spatial spillover), proviene de los elementos fuera de la diagonal principal de (1.17) y, nuevamente, será diferente para cada región. En este caso se tiene una sumatoria de efectos para cada unidad y es este efecto indirecto lo que la literatura ha denominado con diferentes nombres como efecto derrame, contagio o desbordamiento.

En un modelo espacial con la presencia de rezago espacial endógeno, siempre se tendrá efecto marginal que no es único para todas las unidades (en contraposición a lo que sucede bajo un modelo no-espacial). Debido a esta característica, LeSage \& Pace (2009) proponen una medida resumen para cada uno de los efectos considerando los valores promedio:

$$
\begin{aligned}
\bar{M}_{\text {Total }} & =\frac{\beta_{k}}{N} 1_{N}^{\prime} S 1_{N}=\frac{\beta_{k}}{N} \sum_{i} \sum_{j} S_{i, j} . \\
\bar{M}_{\text {Directo }} & =\frac{\beta_{k}}{N} \operatorname{tr}(S)=\frac{\beta_{k}}{N} \sum_{i} S_{i, i} . \\
\bar{M}_{\text {Indirecto }} & =\frac{\beta_{k}}{N} 1_{N}^{\prime} S 1_{N}-\frac{\beta_{k}}{N} \operatorname{tr}(S)=\frac{\beta_{k}}{n} \sum_{i} \sum_{j \neq i} S_{i, j} .
\end{aligned}
$$

La significancia de estos efectos puede ser obtenida mediante simulación Monte Carlo por medio de shocks aleatorios en el término de error. 
Es importante destacar que estos efectos deben ser cuidadosamente interpretados y no están libres de controversias, ya que se tiende a darles un fuerte componente causal. Incluso, algunos autores sugieren que el parámetro espacial del modelo SLM es un parámetro causal, Gibbons \& Overman (2012). Sin embargo, el parámetro autorregresivo espacial captura solo la dinámica espacial de la propia variable endógena y no debe interpretarse como causal ni tampoco los efectos marginales obtenidos ${ }^{7}$.

Por último, cabe mencionar que la evaluación de la bondad de ajuste en los modelos espaciales es un poco más complicada que la habitual. En estos modelos, el uso del $R^{2}$ puede ser engañoso, ya que el $R^{2}$ deja de tener una relación directa con el test de significatividad conjunta (test $\mathrm{F}$ ). Específicamente, los residuos del modelo estimado no tienen media cero y la descomposición estándar de la variabilidad total en variabilidad observada, explicada y variabilidad residual no se mantiene (Anselin, 1988). Con el fin de establecer una comparación de la adecuación de las diversas especificaciones, se presentará un pseudo $-R^{2}$, que mide el grado de correlación al cuadrado entre la variable dependiente observada y la variable predicha, la cual provee una medida de asociación lineal que toma valores entre 0 y 1 . Esta medida no está relacionada con la descomposición de la varianza (Buse, 1973, 1979).

${ }^{7}$ Para ver el motivo de la errónea interpretación puede establecerse un ejemplo paralelo de un proceso temporal $\operatorname{AR}(1), y_{t}=\rho y_{t-1}+\varepsilon_{t}$. No es correcto interpretar a $\rho$ como parámetro causal en series de tiempo. Lo mismo puede decirse para el caso SLM, para mayor detalle sobre este punto véase Herrera et al. (2014). 


\section{Valuación de los riesgos de inundación en La Plata}

\section{Introducción}

En la tarde del 2 de abril de 2013 una gran inundación golpeó la ciudad de La Plata, en la cual murieron 89 personas y miles fueron desplazadas de sus hogares. Ese evento, la inundación más importante de la ciudad en los últimos 100 años, hizo que las autoridades empezaran a considerar la construcción de una nueva infraestructura hidráulica, a desarrollar planes de contingencia y a regular mejor el crecimiento urbanístico de la ciudad. Si bien este tipo de políticas son necesarias, poco se sabe acerca de los beneficios que las personas ven en ellos. A su vez, se produjo un debate en la opinión pública sobre la adjudicación de permisos de construcción. Este trabajo pretende arrojar algo de luz sobre esta cuestión mediante la estimación de la disposición a pagar para evitar el riesgo de sufrir una inundación.

La ocurrencia de inundaciones ha producido importantes costos a nivel mundial, no sólo en términos de las pérdidas materiales y de la interrupción de la actividad económica, sino también en términos de vidas humanas. Los últimos datos disponibles indican que las inundaciones causaron pérdidas monetarias cercanas a los 21 mil millones de dólares durante el primer semestre de 2013, lo cual representa la mayoría de los costos económicos de las catástrofes naturales (Wake, 2013). La mayor parte de los costos económicos durante ese período fueron derivados de las inundaciones en el interior de Europa, Canadá, Asia y Australia. Mientras que las pérdidas económicas son típicamente mayores en los países desarrollados, la mayoría de las muertes por inundaciones ocurren en el mundo en desarrollo ${ }^{1}$

Recientemente, los episodios relacionados con las inundaciones han recibido más atención pública, no solo debido a la mejora de las telecomunicaciones, sino, también, porque los fenómenos meteorológicos extremos se han vuelto cada vez más frecuentes. Según el Grupo Intergubernamental de Expertos sobre el Cambio Climático, las inundaciones y las tormentas

\footnotetext{
${ }^{1}$ Por ejemplo, el desastre de 2013 en Uttarakhand, al norte de India, dio lugar a una cifra cercana a las 6000 personas muertas. Según el último Informe Mundial sobre Desastres Naturales, el número de personas afectadas por las inundaciones ocupa el primer lugar entre los distintos desastres naturales, más que duplicando el número de afectados por otros eventos. La mayoría de los afectados viven en países con niveles de desarrollo humano bajos y medios (IFRC, 2014).
} 
se encuentran entre las mayores amenazas como resultado del calentamiento global (IPCC, $2014)^{2}$.

La rápida urbanización en los países en desarrollo, como Argentina, presentan un desafío adicional al intentar mitigar los riesgos de las inundaciones. Una de las consecuencias de la continua urbanización no planificada es la tendencia de las personas a establecerse en zonas más económicas, en particular, aquellas zonas aledañas a los arroyos con alto riesgo de inundación, lo que incrementa la exposición a inundaciones.

La ciudad de La Plata tiene algunas características atractivas para el propósito de este estudio. En primer lugar, a pesar de que la ciudad está muy cerca del Río de la Plata, no se encuentra en sus orillas. El lote de la muestra más cercano a la orilla del Río de la Plata se encuentra a más de 10 kilómetros ${ }^{3}$. Este hecho permite superar el efecto de confusión que la proximidad a la costa y, en especial, a tener acceso a una vista privilegia, puede tener sobre el riesgo de inundación. La ciudad es atravesada por cinco arroyos cuyas corrientes sirven como afluentes del Río de la Plata. Estos arroyos son responsables por el potencial riesgo de anegamiento de la zona y en sí mismos no tienen valor estético o recreativo. En segundo lugar, La Plata es una ciudad bastante homogénea en términos de topografía y de acceso a servicios ambientales. Por último, la ciudad es relativamente plana, de baja altitud, y muy cercana a un gran río. Según las proyecciones de IPCC (2014) sobre el crecimiento del nivel del mar y las tormentas intensas en la zona, debido al cambio climático los episodios de inundación serán más probables en el futuro.

Los resultados obtenidos indican que el valor de mercado de un inmueble situado en una zona propensa a inundaciones es menor al de una propiedad similar localizada fuera de dicha zona. La reducción en el precio de las propiedades en zonas propensas a inundaciones es de, aproximadamente, un $12 \%$.

El capitulo se organiza de la siguiente manera: en la sección siguiente se realiza la revisión de la literatura y en la segunda sección se presentan los datos utilizados. La tercera sección expone la estrategia empirica y la cuarta sección detalla los principales resultados. Se exponen las conclusiones en la última sección.

\footnotetext{
${ }^{2}$ En particular, el IPCC afirma que los riesgos asociados con el cambio climático a partir de los fenómenos extremos, como las olas de calor, precipitaciones extremas y las inundaciones costeras, ya son moderadas (confianza alta) y alta con un grado centigrado adicional (confianza media). En consecuencia, las pérdidas económicas se prevén que aumenten debido a las inundaciones urbanas en las próximas décadas (Hallegatte et al., 2013).

${ }^{3}$ La cuenca del Plata es una de las más importantes del mundo, el drenaje es de aproximadamente una quinta parte del continente sudamericano. Con una anchura máxima de 220 kilómetros, la cuenca del Río de la Plata es considerada como la más ancha del mundo.
} 


\subsection{Revisión de la literatura}

Desde el trabajo pionero de Lancaster (1966) y Rosen (1974), muchos estudios han aplicado el modelo de precios hedónicos para conocer la relación entre los precios de los inmuebles y los riesgos medioambientales, entre los que se encuentran las inundaciones, los terremotos o la cercanía a plantas químicas. Los modelos de precios hedónicos permiten inferir la disposición a pagar para reducir la exposición a los diversos riesgos mediante la identificación del valor total de la probabilidad de que tales riesgos efectivamente se materialicen, incluyendo no sólo las pérdidas materiales, sino, también, las pérdidas no materiales y subjetivas. Varios estudios hedónicos, principalmente para los Estados Unidos, han abordado específicamente el tema de las inundaciones (Park \& Miller, 1982; Thompson \& Stoevener, 1983; Donnelly, 1998; Speyrer \& Ragas, 1991; Harrison et al., 2001; Zhai et al., 2003; Bin \& Polasky, 2004; Bin \& Kruse, 2006; Samarasinghe \& Sharp, 2010; Kousky, 2010; Carbone et al., 2006; Bin et al., 2008 por nombrar algunos). Aunque estos trabajos suelen diferir en su definición del riesgo de inundación, la gran mayoría encuentra un descuento en el precio estadísticamente significativo. En general, una propiedad ubicada en un lugar inundable tiene un descuento en el precio de, aproximadamente, un $5 \%$ en relación a una propiedad similar en una zona de bajo riesgo ${ }^{4}$. Un meta-análisis sobre 19 estudios de los Estados Unidos informa que un aumento en la probabilidad de inundación de un $1 \%$ en un año se traduce en una disminución de $0,6 \%$ en los valores de transacción de las propiedades (Daniel et al., 2009).

La literatura ha cubierto otras cuestiones relacionadas con riesgos de inundación además de su impacto directo sobre el valor de la vivienda. Por ejemplo, el efecto del seguro de inundación ha sido objeto de atención en diversos estudios empíricos ${ }^{5}$. En este caso, el descuento en el precio asociado a estar localizado en un lugar inundable se compara con el valor capitalizado de la prima del seguro contra inundaciones. En general, los resultados muestran que el descuento por el riesgo de inundación a menudo excede la capitalización de la prima de seguro, lo que implica que algunos riesgos no están completamente asegurados (por ejemplo, las pérdidas no monetarias $)^{6}$. Una explicación alternativa es la existencia de información incompleta por parte de los propietarios sobre los verdaderos riesgos de inundación, o una subestimación de los costos asociados a las inundaciones. Esta interpretación se ve reforzada por los resultados de encuestas que muestran que una gran proporción de hogares desconocen los riesgos de inundación, sobre todo cuando no se han producido inundaciones desde hace algún tiempo. Encuestas en Boulder, Colorado, USA revelan que la mayoría de los propietarios de viviendas en zonas inundables carecían de información sobre el riesgo de inundación cuando compraron una casa, y el $70 \%$

\footnotetext{
${ }^{4}$ Las zonas inundables, por lo general, se definen como aquellas áreas de los terrenos adyacentes a los cursos de agua que serían desbordados durante un evento de inundación que tiene un $1 \%$ de probabilidad de ocurrencia en cada año.

${ }^{5}$ Estos estudios son principalmente para Estados Unidos, donde la Agencia Federal de Manejo de Emergencias (FEMA) requiere un seguro obligatorio en las zonas propensas a inundaciones. Sin embargo, en Argentina no existen seguros de ese tipo.

${ }^{6}$ Una excepción es Harrison et al. (2001).
} 
de los encuestados dijeron que hubiesen reducido su oferta si hubieran sabido el costo actual del seguro contra inundaciones (Chivers \& Flores, 2002). En el Reino Unido, la encuesta de la Agencia de Medio Ambiente (DEFRA, 2008) sobre hogares en las zonas inundadas en 1998 y 2000 reveló que, antes de que ocurrieran las inundaciones, sólo el $24 \%$ de los hogares eran conscientes del riesgo de inundación (Burningham et al., 2008). Además, la encuesta de 2005 del British Market Research Bureau estimó que el $41 \%$ de las 5 millones de personas (2 millones de propiedades) que viven en Inglaterra y en Gales sobre zonas de riesgo de inundación no tenían conocimiento de la amenaza de inundación (BMRBB, 2005).

Algunos trabajos han estimado el impacto de una inundación particular sobre los valores inmobiliarios. Bin \& Polasky (2004) encuentran un descuento en el precio para viviendas ubicadas en zonas propensas a inundaciones después del huracán Floyd. Zhai et al. (2003) reportan un resultado similar después de una gran inundación en el centro de Japón. La ventaja de este enfoque es que permite inferir cómo los participantes del mercado actualizan los riesgos percibidos inmediatamente después de tal evento. Sin embargo, si estos valores reportados se mantienen o no en el tiempo es otra cuestión. Kousky (2010) utiliza casi 30 años de ventas de propiedades en St. Louis, Missouri, para estimar no sólo el impacto inmediato de una gran inundación, sino, también, cómo los riesgos percibidos varían en los años posteriores al evento.

De acuerdo con esta línea de la literatura, algunos trabajos como Tobin \& Newton (1986) y Tobin \& Montz (1994) argumentan que el descuento en el precio de los inmuebles en zonas inundables depende de la frecuencia con que se producen inundaciones en comparación con el tiempo necesario para restaurar la propiedad. En un extremo con inundaciones esporádicas, los precios inmobiliarios caen inmediatamente después de un evento de inundación y luego se recuperan completamente después de las reparaciones y permanecen en este nivel más alto hasta la próxima inundación. En este caso, en el largo plazo, ni los daños por inundación pasados ni futuros son capitalizados. En el otro extremo, con inundaciones que ocurren frecuentemente, los precios de las viviendas no tienen tiempo suficiente para recuperarse, por lo que los precios de las propiedades siguen siendo bajos. En este caso, los daños de las inundaciones han sido casi totalmente capitalizados en los precios de las casas ex post facto. Los casos de frecuencias de inundación intermedias tendrían un patrón intertemporal de los precios de la vivienda con una capitalización incompleta e imperfecta de los daños por inundación.

Otra rama de la literatura analiza el efecto de las regulaciones urbanísticas en zonas propensas a inundaciones sobre la percepción del riesgo y, en última instancia, en los valores inmobiliarios. En particular, estas regulaciones implican la divulgación de información sobre los riesgos de inundación de cada zona. Dado que muchas personas pueden no ser plenamente conscientes de que viven en una zona propensa a inundaciones, se ha hecho común, al menos en los países desarrollados, exigir que los compradores potenciales sean informados sobre las probabilidades reales de sufrir una inundación. El efecto de este tipo de regulaciones depende, en última instancia, de qué tan diferentes sean los riesgos objetivos de los riesgos percibidos por los 
propietarios y por los compradores potenciales. Por ejemplo, Speyrer \& Ragas (1991) concluyen que el descuento en el precio se produce por la publicidad de la información sobre dichos peligros.

\subsection{Datos utilizados}

La ciudad de La Plata es la capital de la provincia de Buenos Aires. Según el censo de 2010, tiene una población de 649.613 habitantes en 265.677 hogares. La Plata es una ciudad planificada, un paradigma de planificación urbana de finales del siglo XIX ${ }^{7}$. El plano original de la ciudad fue diseñado por el arquitecto Pedro Benoit y se caracterizaba por una red de líneas rígidas que llevaban a importantes avenidas. Hoy en día, la ciudad se ha expandido a varios suburbios sin mucha planificación urbana ${ }^{8}$ La Plata está situada en la parte noreste de la provincia de Buenos Aires, a unos 60 kilómetros al sur de la ciudad de Buenos Aires.

\subsubsection{Mercado de Lotes baldíos}

Los datos utilizados incluyen 679 lotes puestos a la venta durante el año $2004^{9}$. Los datos provienen de lotes en oferta anunciados por 28 agencias inmobiliarias locales. Estas inmobiliarias, que representan alrededor del $95 \%$ del mercado inmobiliario local, publican sus ofertas en un sistema de información en línea llamado SIOC (Sistema Inmobiliario de Ofertas por Computación). Este sistema proporciona información sobre las propiedades en venta como la ubicación exacta de la propiedad, su precio de oferta, la dimensión de la parcela y, también, contiene información sobre la disponibilidad de servicios públicos en la zona, como el acceso a cloacas y al suministro de agua corriente. Además de la información obtenida del SIOC, se calculó la distancia hasta el centro comercial de la ciudad ${ }^{10}$. También se buscó para cada lote el factor de ocupación total del suelo permitido por el municipio (FOT) para cada lote.

También se tuvieron en cuenta los efectos de los barrios de La Plata. Se incluyó un conjunto de variables dummy para cada barrio o localidad. El propósito de estas variables ficticias es capturar las diferencias socioeconómicas no observables entre los distintos barrios ${ }^{11}$.

\footnotetext{
${ }^{7}$ La ciudad fue galardonada con dos medallas de oro en las categorías Ciudad del Futuro y Mejor Realización Construida en la Exposición Universal de 1889 en París.

${ }^{8} \mathrm{El}$ área metropolitana de La Plata incluye las ciudades de Tolosa, Ringuelet, Gonnet, City Bell, Villa Elisa, Melchor Romero, Abasto, Gorina, José Hernández, Ángel Etcheverry, Arturo Segui, Los Hornos, Lisandro Olmos, Villa Elvira y Altos de San Lorenzo, todos ellos con centros comunales que funcionan como oficinas del gobierno local.

${ }^{9} \mathrm{No}$ fueron consideradas las parcelas ubicadas en barrios cerrados, ya que en la determinación de su precio están implicados otros factores como los servicios de seguridad, las instalaciones deportivas y recreativas e, incluso, la ubicación del lote dentro del emprendimiento urbanístico cerrado.

${ }^{10} \mathrm{El}$ centro comercial de la ciudad se estableció en la intersección de las calles 8 y 47 . Los cálculos se realizaron con el comando traveltime en Stata, que calcula las distancias de viaje y el tiempo en diversos medios de transporte a través de Google Maps.

${ }^{11}$ En esencia, las dummys por barrio capturan las diferencias en los índices delictivos y en la calidad de la infraestructura de transporte.
} 
Adicionalmente, se generó una variable dicotómica que indica si el lote en cuestión se encuentra sobre una avenida. Es importante remarcar la escasa disponibilidad de lotes en el trazado original de la ciudad, que representa sólo el $16 \%$ de las parcelas de la muestra.

Antes de analizar los datos, es necesario aclarar que no fue posible acceder a datos de transacciones inmobiliarias reales y por esta razón se trabajó con precios de oferta. Si bien el mercado inmobiliario formal se encuentra bien desarrollado en la Argentina, los precios de transacción son casi siempre sub reportados con el fin de evitar el pago de los impuestos correspondientes. Los valores de las propiedades compradas mediante un crédito hipotecario por lo general se registran con el valor de transacción real, ya que hay institución financiera formal involucrada. Sin embargo, estas operaciones son poco comunes en el mercado inmobiliario argentino. De acuerdo con la Asociación de Bancos, mientras que en Chile los créditos hipotecarios en 2012 representaron casi el 18\% del producto interno bruto y en Brasil el 6,8\%, en Argentina sólo alcanzó el 1,5\%. Por lo tanto, no se mejoraría el análisis considerando los valores de las transacciones declarados en lugar de los precios de oferta en el mercado inmobiliario $\operatorname{argentino}^{12}$. No obstante, los precios de oferta, aunque potencialmente diferentes de los valores de equilibrio, se establecen principalmente por tasadores experimentados, lo que lleva a suponer que no deberían ser muy diferentes de los valores de equilibrio. Por otra parte, la relativamente alta actividad económica durante el período analizado (el PIB de Argentina creció alrededor de $9 \%$ por año entre 2003 y 2005) sugiere que los oferentes vendieron sus propiedades muy a menudo por el precio de venta, lo que implica que los precios deben haber estado cerca del valor real de la transacción. El mercado inmobiliario argentino no se caracteriza por negociaciones de precios significativas entre compradores y vendedores. En general, las propiedades inmobiliarias se venden por su precio de venta y, en el caso de que una propiedad no se venda rápidamente después de ser puesta a la venta, por lo general se prefiere no disminuir el precio, sino mantener la casa en el mercado por un período más largo. Incluso en los mercados de vivienda más sofisticados, como en los Estados Unidos, donde compradores y vendedores se involucran más en las negociaciones, una proporción significativa de las propiedades se vende a su precio de oferta. Por ejemplo, Case \& Shiller (2003) informan que 47,4\% de los inmuebles en cuatro grandes ciudades de Estados Unidos (Los Ángeles, San Francisco, Boston y Milwaukee) se vendieron por su precio de oferta.

Los lotes baldíos tienen la ventaja de ser propiedades más homogéneas que las casas y los departamentos, ya que no poseen una construcción que afecte su precio. En particular, los únicos atributos relevantes de los terrenos son sus dimensiones, el acceso a los servicios y la ubicación. El cuadro 2.1 presenta las estadísticas descriptivas de los lotes considerados. El precio medio de los lotes es de 21864 dólares, tienen en promedio una dimensión de 600 metros

\footnotetext{
${ }^{12}$ Sin embargo, la subdeclaración (no observada) no sería un problema para el ejercicio empírico si en la práctica se aplicase de manera uniforme en todo el mercado, es decir, si se informarse el precio de transacción de cada inmueble con un descuento de, por ejemplo, un $10 \%$. Sin embargo, no hay evidencia de que tal subdeclaración se realice de manera uniforme en todo el mercado.
} 
cuadrados y se encuentran en zonas con buen acceso a los servicios públicos, con excepción de cloacas y de pavimento. El 16\% de los lotes se encuentra sobre una avenida y la distancia promedio al centro comercial es de poco más de 7 kilómetros con un máximo de 17 kilómetros.

Al analizar los datos por barrio se encuentran algunas diferencias importantes, las cuales se muestran en el cuadro 2.2. La localidad de Hernández y el barrio Aeropuerto contienen los lotes más baratos, con valores promedio cercanos a los 8 mil dólares, mientras que en el casco urbano se encuentran los lotes más caros, con valores promedio superiores a los 61 mil dólares. Siguiendo en orden descendente se encuentran las localidades de Gonnet (18485), City Bell (18279), Tolosa (16888) y Ringuelet (16123). En lo que se refiere al tamaño de los lotes, los de mayor tamaño se encuentran en las localidades de Gonnet, Villa Elisa, City Bell y San Carlos con terrenos de 700 metros cuadrados, mientras que los más pequeños, con pocos más de 400 metros se encuentran en Los Hornos y en Tolosa. En lo que respecta al FOT, solo en el casco urbano se encuentran valores superiores a la unidad, con valores promedio de 2.21.

\subsubsection{Riesgos de Inundación}

El clima en la región donde se encuentra la ciudad de La Plata es bastante húmedo durante todo el año debido a su proximidad a la costa. La humedad media es superior al $75 \%$ durante todo el año. En la ciudad caen alrededor de $1100 \mathrm{ml}$ de lluvia cada año, siendo los inviernos los meses más secos y los veranos los más húmedos ${ }^{13}$.

Los datos sobre el riesgo de inundación provienen de un estudio del Instituto de Geomorfología y Suelos de la Universidad Nacional de La Plata realizado durante la década de los 2000, Hurtado et al. (2006). Dicho informe está basado en el análisis de la geomorfología del terreno y de las escorrentías de la zona y permite identificar las zonas propensas a inundarse en el partido de La Plata. Desde el punto de vista físico, las zonas inundables son las áreas más propensas a verse afectadas por las lluvias y por los desbordes de los cuerpos de agua, que a su vez influyen en la posición relativa de la capa freática ${ }^{14}$, lo que reduce la capacidad de almacenamiento subterráneo del agua. La mayor parte de la ciudad de La Plata es plana y de baja altitud. El punto de mayor elevación se encuentra a sólo 24 metros sobre el nivel del mar y cerca del $85 \%$ se compone de suelo que posee mal drenaje.

Con el fin de vincular los riesgos de inundación a los datos de las propiedades utilizadas fue necesario georeferenciar cada inmueble en la muestra, un proceso que se llevó a cabo de forma manual utilizando la dirección postal. Luego, a cada observación se le asignó una de las tres categorías de riesgo de inundación (riesgo bajo, moderado y alto) elaboradas por el Instituto

\footnotetext{
${ }^{13}$ Para tener una idea de lo dramático de la inundación de abril 2013, la cantidad de lluvia que cayó en menos de 5 horas fue el equivalente al $20 \%$ de las precipitaciones anuales (cerca de tres veces el nivel normal de abril).

${ }^{14} \mathrm{El}$ nivel freático o la tabla de agua es la distancia a la que se encuentra el agua desde la superficie del terreno.
} 
de Geomorfologia y Suelos utilizando el software ArcGIS ${ }^{15}$. La Figura 2.1 muestra el Área Metropolitana de La Plata, los arroyos, las diferentes áreas propensas a inundaciones y los lotes utilizados en el análisis. En las líneas azul oscuro se muestran las escorrentías de la ciudad, las zonas color azul claro son aquellas en las que el riesgo de inundación es más alto, con una recurrencia de una inundación cada 20 años. Estas áreas incluyen los márgenes de los arroyos, zanjas, llanuras de barro y estuarios interiores. Alrededor del $22 \%$ de los terrenos baldíos se encuentra en zonas de alto riesgo. Las regiones sombreadas en azul que rodean las zonas de alto riesgo son aquellas zonas donde los riesgos se clasifican como moderados e incluyen las pendientes que convergen hacia los arroyos. Es posible que algunos lotes en una zona de riesgo de inundación moderada nunca hubieran experimentado una inundación hasta abril de 2013. Alrededor del $27 \%$ de los lotes se encuentra dentro de estas áreas. El riesgo está asociado a una inundación entre 20 y 100 años. El resto de las parcelas, 51\%, enfrenta nivel de riesgo de inundación bajo ${ }^{16}$. El riesgo asociado en esta clasificación es una inundación cada 100 años.

$\mathrm{Al}$ analizar los riesgos de inundación por localidad, se puede observar en el cuadro 2.2 que las localidades de Gonnet (68\%) y de Ringuelet (87\%) son las que tienen una mayor proporción de los lotes en venta sobre zonas con riesgo de inundación, y de esos lotes el $66 \%$ en Gonnet y el $47 \%$ en Ringuelet corresponden a zonas con alto riesgo de inundación. En cambio, en las zonas del barrio Aeropuerto y Hernández solo el $22 \%$ de las propiedades en venta se encuentra en zonas con riesgo hídrico y solo el $2 \%$ se encuentra en zonas con alto riesgo. Sin embargo, las zonas de Aeropuerto y Hernández son las menos demandadas.

\subsection{Estrategia empírica}

Como se especificó en el capítulo metodológico, se utiliza un enfoque de precios hedónicos espaciales para estimar el efecto que tiene sobre los valores de los lotes baldíos el hecho de que estén localizados en lugares propensos a inundaciones.

La estrategia empírica comienza con la estimación por MCO de un modelo hedónico que relaciona el precio de un inmueble con las características del lote, la calidad ambiental, el riesgo de inundación y las características socioeconómicas del barrio. Formalmente, se estima el siguiente modelo:

$$
P=X \beta+F \gamma+\mu,
$$

${ }^{15}$ En realidad, los riesgos de inundación varían dentro de la llanura de inundación, por lo general disminuye con la distancia al arroyo o al río. Sin embargo, la mayoría de los estudios empíricos establecen los riesgos de inundación como una variable dicotómica, es decir, si la propiedad se encuentra o no en la llanura de inundación. Excepciones notables en la literatura son Bartosova et al. (2000) y Rambaldi et al. (2013), cuyos trabajos utilizan una medida del riesgo de inundación continua.

${ }^{16}$ Algunas de estas propiedades se inundaron en realidad durante el evento 2013. Ese evento fue, sin embargo, realmente único en la historia de la ciudad. 
donde $P$ es un vector de $N$ por 1 que contiene los precios de las propiedades, $X$ es una matriz de $N$ por $K$ de variables independientes referidas a la dimensión de la parcela, la disponibilidad de servicios públicos en la zona, como el acceso a cloacas, suministro de agua corriente, factor de ocupación total del suelo, la distancia al centro comercial y si el lote en cuestión se encuentra sobre una avenida, junto con las dummys por barrio y las características socioeconómicas de la zona. $F$ es una matriz de $N$ por $L$ variables indicadoras del riesgo de inundación y $\mu$ es el término de error.

Luego, se realizan los test de Lagrange propuestos por Anselin et al. (1996) sobre los errores de la estimación por MCO para detectar la existencia de interacciones espaciales. Los resultados de los test obtenidos en el cuadro 2.3, muestran que tanto los multiplicadores de Lagrange $L M_{L a g}$ y $L M_{\text {Error }}$, como sus versiones robustas son significativos para casi todas las especificaciones analizadas y, por lo tanto, sugieren la inclusión de ambos tipos de interacciones espaciales.

El modelo a estimar será un modelo hedónico espacial SARAR que considera no sólo la dependencia espacial en la variable dependiente, sino, también, que los errores provengan de un proceso autorregresivo espacial. Formalmente, se estima el siguiente modelo:

$$
P=\rho W P+X \beta+F \gamma+\mu
$$

con

$$
\mu=\lambda W \mu+\varepsilon
$$

donde $P, X$ y $F$ han sido definidos anteriormente, con $\rho$ como el coeficiente de la variable dependiente rezagada espacialmente y $\lambda$ como el coeficiente de la estructura autorregresiva espacial de la perturbación $\mu$. El coeficiente $\rho$ muestra la intensidad de la dependencia especial e indica cómo afectan los cambios en una propiedad a las propiedades vecinas. Un valor alto del indicador indica efectos derrame más altos (mayores efectos indirectos). El coeficiente $\lambda$ mide la intensidad de la heterogeneidad espacial y muestra la intensidad de las relaciones espaciales en los errores. El coeficiente lambda indica que un shock aleatorio afectará no sólo a la región en donde se provocó, sino que se transmitirá a todo el sistema. El valor del coeficiente representa la intensidad con la que ese shock se transmite a lo largo del espacio.

La matriz $W$, de dimensión $\mathrm{N}$ por $\mathrm{N}$, es la matriz de ponderadores espaciales obtenida aplicando el criterio de distancia según la función definida en el capítulo 1. Las innovaciones $\varepsilon$ se asumen independientes e idénticamente distribuidas (i.i.d.) o independientes pero heterocedasticas, donde la heteroscedasticidad es de una forma desconocida. 


\subsection{Resultados}

Los resultados del análisis sobre cómo los riesgos de inundación impactan en los precios de los terrenos baldíos se presentan en el cuadro 2.4, así como también los coeficientes que acompañan las otras características de los lotes.

La variable dependiente es el logaritmo natural del precio de venta. Las dos primeras columnas corresponden a estimaciones por mínimos cuadrados ordinarios (MCO) mientras que las restantes columnas muestran las estimaciones de los modelos espaciales realizadas por el Método de Momentos Generalizados (GMM). El cuadro 2.5 presenta los efectos marginales de las variables del riesgo de inundación para los modelos espaciales.

La ubicación en una zona con riesgo hídrico afecta negativamente al precio, las estimaciones de MCO (columna 1) muestran un descuento estadísticamente significativo en el precio de alrededor del 8,5\%. Las estimaciones de los modelos espaciales sugieren un descuento aproximado del $7 \%$ en el precio (ver efectos marginales en el Panel A del cuadro 2.5). Al desagregar los riesgos de acuerdo con su intensidad, las parcelas en zonas de mayor riesgo sufren un descuento significativo y de mayor tamaño, del 12,7\% en el modelo por MCO (columna 2) y entre el 11,7\% y el 13,3\% en los modelos espaciales (Panel B del cuadro 2.5). Sin embargo, el mercado no parece discriminar riesgos moderados de situaciones de bajo riesgo (Panel $\mathrm{C}$ del cuadro 2.5).

Los descuentos estimados para los lotes baldíos son superiores a los obtenidos para lotes con construcciones encontrados en la literatura. Los resultados sugieren que el mercado percibe los lotes como más riesgosos. Por un lado, podría ser que los lotes se encuentren en zonas de mayor riesgo y, por lo tanto, los riesgos percibidos estén alineados con los riesgos objetivos diferenciales. A su vez, el hecho de que la ciudad se esté expandiendo hacia áreas propensas a inundaciones proporciona alguna evidencia en esa dirección. Por otro lado, un descuento superior podría ser totalmente debido a razones subjetivas.

Tanto el coeficiente $\rho$ que mide dependencia espacial, como el coeficiente $\lambda$ que mide la estructura autorregresiva espacial de los errores tienen valores significativos. El resto de las características de los lotes tienen el efecto esperado. A medida que los lotes son más grandes, tienen mejor acceso a infraestructura y a servicios públicos y tienen precios mayores. Las parcelas que se encuentran más lejos del centro tienen un menor valor y los lotes que se encuentran ubicados sobre avenidas tienen un precio mayor. El factor de ocupación del lote, el cual determina la cantidad de metros que pueden construirse, tiene un efecto positivo sobre el precio del mismo. Por último, las características socioeconómicas de la zona, como el ingreso de los hogares y la tasa de desempleo, potencialmente correlacionadas con los atributos de vecinos, tienen el efecto esperado en los precios inmobiliarios. 


\subsection{Conclusiones}

La mitigación de los riesgos de inundación ha ido ganando la atención de la opinión pública debido a que se prevé que los fenómenos meteorológicos extremos se intensifiquen en un futuro cercano. Existe una amplia literatura empírica sobre la disposición a pagar para evitar los riesgos de inundación en zonas urbanas, pero se centra casi por completo en los países desarrollados. La evidencia de los países en desarrollo es escasa, particularmente para Latinoamérica.

En este capitulo se ha estimado el efecto que los riesgos de inundación tienen sobre los precios de los terrenos baldíos utilizando modelos hedónicos espaciales para la ciudad de La Plata. Los resultados indican que cuando las propiedades se encuentran en zonas con riesgo hídrico sufren un descuento en el precio. Las estimaciones muestran que los lotes en zonas propensas a inundaciones son aproximadamente un $12 \%$ más baratos. Los descuentos estimados para los lotes baldíos son superiores a los obtenidos en la literatura para lotes con edificaciones, lo que sugiere que el mercado percibe a los lotes baldíos como más riesgosos.

Una política destinada a proporcionar información a los potenciales compradores sobre la exposición al riesgo sin duda aumentaría el bienestar general. Se considera que este estudio tiene importantes implicancias en las decisiones sobre la realización de inversiones en infraestructura. La disponibilidad a pagar estimada debe ser confrontada con los costos esperados de los distintos proyectos de control de inundaciones con el fin de priorizar las inversiones.

Resulta sumamente interesante analizar cómo el descuento en el precio por estar localizado en una zona con riesgo hídrico cambió después de la inundación de 2013. Es muy probable que la gente haya actualizado su percepción del riesgo y, por lo tanto, la reducción de los precios inmobiliarios sea mayor. Sin embargo, se deja esta tarea para futuras investigaciones. 


\subsection{Apéndice}

Figura 2.1: Zonas propensas a inundaciones y ofertas inmobiliarias en La Plata

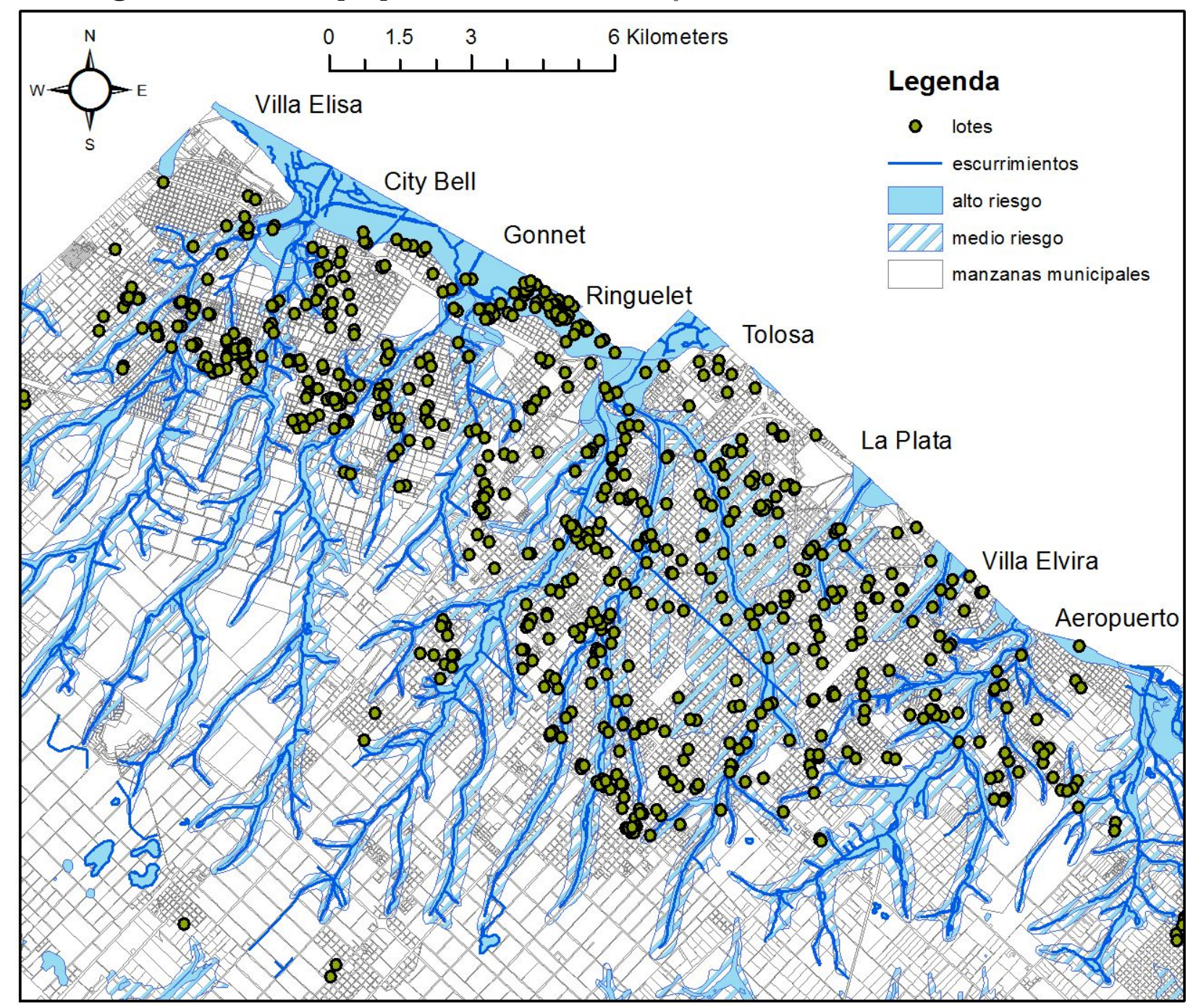




\section{Cuadro 2.1: Estadistas Descriptivas}

\begin{tabular}{|c|c|c|c|c|c|}
\hline Descripción & Media & Desvío estándar & Mínimo & Máximo & Fuente \\
\hline Valor del lote (Dólares 2004) & 21864 & 28925 & 1067 & 279067 & $\mathrm{f} 1$ \\
\hline Valor del lote (Dólares por m2) & 44 & 55 & 2 & 464 & f1 \\
\hline Superficie del terreno (metros cuadrados) & 600 & 541 & 128 & 4644 & f1 \\
\hline Fondo (en metros) & 39 & 17 & 13 & 220 & f1 \\
\hline Frente (en metros) & 15 & 9 & 6 & 78 & f1 \\
\hline Factor de ocupación total & 1.11 & 0.70 & 0.60 & 3.00 & f1 \\
\hline Gas & $72 \%$ & $45 \%$ & $0 \%$ & $100 \%$ & f1 \\
\hline Energía eléctrica & $67 \%$ & $47 \%$ & $0 \%$ & $100 \%$ & f1 \\
\hline Agua de red & $76 \%$ & $42 \%$ & $0 \%$ & $100 \%$ & f1 \\
\hline Cloacas & $53 \%$ & $50 \%$ & $0 \%$ & $100 \%$ & f1 \\
\hline Pavimento & $50 \%$ & $50 \%$ & $0 \%$ & $100 \%$ & f1 \\
\hline Sobre avenida & $16 \%$ & $37 \%$ & $0 \%$ & $100 \%$ & f1 \\
\hline Distancia al centro $(\mathrm{km})$ & 7.10 & 3.70 & 0.50 & 17.00 & $f 2$ \\
\hline Riesgo de inundación: bajo & $51 \%$ & $50 \%$ & $0 \%$ & $100 \%$ & f3 \\
\hline Riesgo de inundación: moderado & $27 \%$ & $44 \%$ & $0 \%$ & $100 \%$ & f3 \\
\hline Riesgo de inundación: alto & $22 \%$ & $41 \%$ & $0 \%$ & $100 \%$ & f3 \\
\hline Salario horario medio & 509 & 49 & 435 & 646 & $\mathrm{f} 4$ \\
\hline Tasa de desempleo & $24 \%$ & $8 \%$ & $8 \%$ & $46 \%$ & $\mathrm{f} 4$ \\
\hline Años de la educación & 4 & 1 & 3 & 6 & $\mathrm{f} 4$ \\
\hline Proporción de propietarios de la vivienda y del terreno & $76 \%$ & $10 \%$ & $33 \%$ & $91 \%$ & $\mathrm{f} 4$ \\
\hline Proporción de propietarios de la vivienda solamente & $4 \%$ & $6 \%$ & $0 \%$ & $55 \%$ & $\mathrm{f} 4$ \\
\hline Proporción de inquilinos & $9 \%$ & $9 \%$ & $1 \%$ & $58 \%$ & $\mathrm{f} 4$ \\
\hline Proporción de ocupantes por relación de dependencia & $7 \%$ & $3 \%$ & $1 \%$ & $31 \%$ & $\mathrm{f} 4$ \\
\hline Proporción de ocupantes por préstamo & $1 \%$ & $3 \%$ & $0 \%$ & $32 \%$ & $\mathrm{f} 4$ \\
\hline Proporción de otra situación & $3 \%$ & $2 \%$ & $0 \%$ & $22 \%$ & $\mathrm{f} 4$ \\
\hline Al menos una NBI & $14 \%$ & $12 \%$ & $0 \%$ & $66 \%$ & $\mathrm{f} 4$ \\
\hline
\end{tabular}

Nota: Los precios de los inmuebles son en dólares estadounidenses. Las fuentes son f1: Sistema Inmobiliario de Ofertas por Computacion (SIOC); f2: SIOC y herramientas geoespaciales; f3: Censo 2001 a nivel de radio censal; f4: Instituto de Geomorfologia y Suelos y herramientas geoespaciales. 


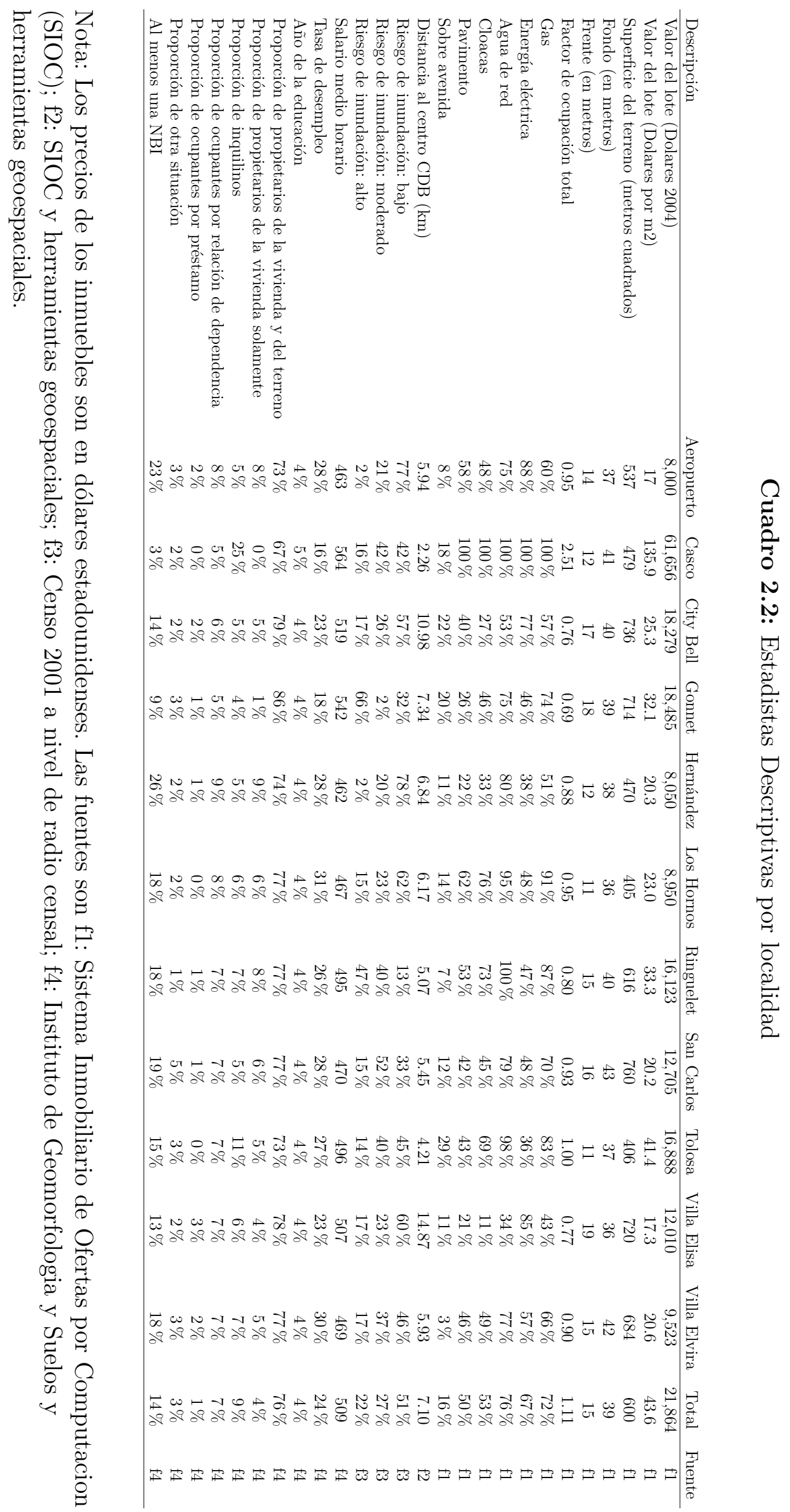


Cuadro 2.3: Diagnostico espacial

\begin{tabular}{lcccc}
\hline \multirow{2}{*}{ Modelo } & \multirow{2}{*}{ I de Moran } & \multicolumn{2}{c}{ Estadístico } \\
\cline { 3 - 4 } & & & Multiplicador de Lagrange & Multiplicador de Lagrange robusto \\
\hline \multirow{2}{*}{1} & Spatial error & \multirow{2}{*}{$8.203^{* * *}$} & $54.493^{* * *}$ & $5.813^{* *}$ \\
& Spatial lag & & $66.098^{* * *}$ & $17.418^{* * *}$ \\
\hline \multirow{2}{*}{2} & Spatial error & $6.709^{* * *}$ & $34.415^{* * *}$ & $5.988^{* *}$ \\
& Spatial lag & & $34.979^{* * *}$ & $6.552^{* *}$ \\
\hline \multirow{2}{*}{3} & Spatial error & $6.71^{* * *}$ & $34.523^{* * *}$ & 2.213 \\
& Spatial lag & & $49.009^{* * *}$ & $16.699^{* * *}$ \\
\hline \multirow{2}{*}{4} & Spatial error & $6.264^{* * *}$ & $29.479^{* * *}$ & $3.795^{*}$ \\
& Spatial lag & & $34.164^{* * *}$ & $8.481^{* * *}$ \\
\hline \multirow{2}{*}{5} & Spatial error & $6.264^{* * *}$ & $29.254^{* * *}$ & $3.843^{*}$ \\
& Spatial lag & & $33.392^{* * *}$ & $7.98^{* * *}$ \\
\hline \multirow{2}{*}{6} & Spatial error & $8.104^{* * *}$ & $52.856^{* * *}$ & $4.844^{* *}$ \\
& Spatial lag & & $66.913^{* * *}$ & $18.901^{* * *}$ \\
\hline \multirow{2}{*}{7} & Spatial error & $6.656^{* * *}$ & $33.64^{* * *}$ & $4.844^{* *}$ \\
& Spatial lag & & $35.467^{* * *}$ & $18.901^{* * *}$ \\
\hline \multirow{2}{*}{8} & Spatial error & $6.643^{* * *}$ & $33.64^{* * *}$ & $5.343^{* *}$ \\
& Spatial lag & & $35.467^{* * *}$ & $7.17^{* * *}$ \\
\hline \multirow{2}{*}{9} & Spatial error & $6.218^{* * *}$ & $33.579^{* * *}$ & $3.298^{*}$ \\
& Spatial lag & & $34.728^{* * *}$ & $9.193^{* * *}$ \\
\hline \multirow{2}{*}{10} & Spatial error & $6.213^{* * *}$ & $28.558^{* * *}$ & $3.342^{*}$ \\
& Spatial lag & & $33.869^{* * *}$ & $8.652^{* * *}$ \\
\hline
\end{tabular}

* significativo al $10 \% ; * *$ significativo al $5 \%$; $* *$ significativo al $1 \%$

El modelo 1 incluye controles por riesgo hídrico (riesgo alto y moderado). El modelo 2 incluye controles por riesgo hídrico, salario medio y proporción de propietarios. El modelo 3 incluye controles por riesgo hídrico, tasa de desocupación y proporción de propietarios. El modelo 4 incluye controles por riesgo hídrico, salario medio y tasa de desocupación. El modelo 5 incluye controles por riesgo hídrico, salario medio, tasa de desocupación y proporción de propietarios. El modelo 6 incluye controles por riesgo de inundación alto y moderado. El modelo 7 incluye controles por riesgo de inundación alto y moderado, salario medio y proporción de propietarios. El modelo 8 incluye controles por riesgo de inundación alto y moderado, tasa de desocupación y proporción de propietarios. El modelo 9 incluye controles por riesgo de inundación alto y moderado, salario medio y tasa de desocupación. El modelo 10 incluye controles por riesgo de inundación alto y moderado, salario medio, tasa de desocupación y proporción de propietarios. 


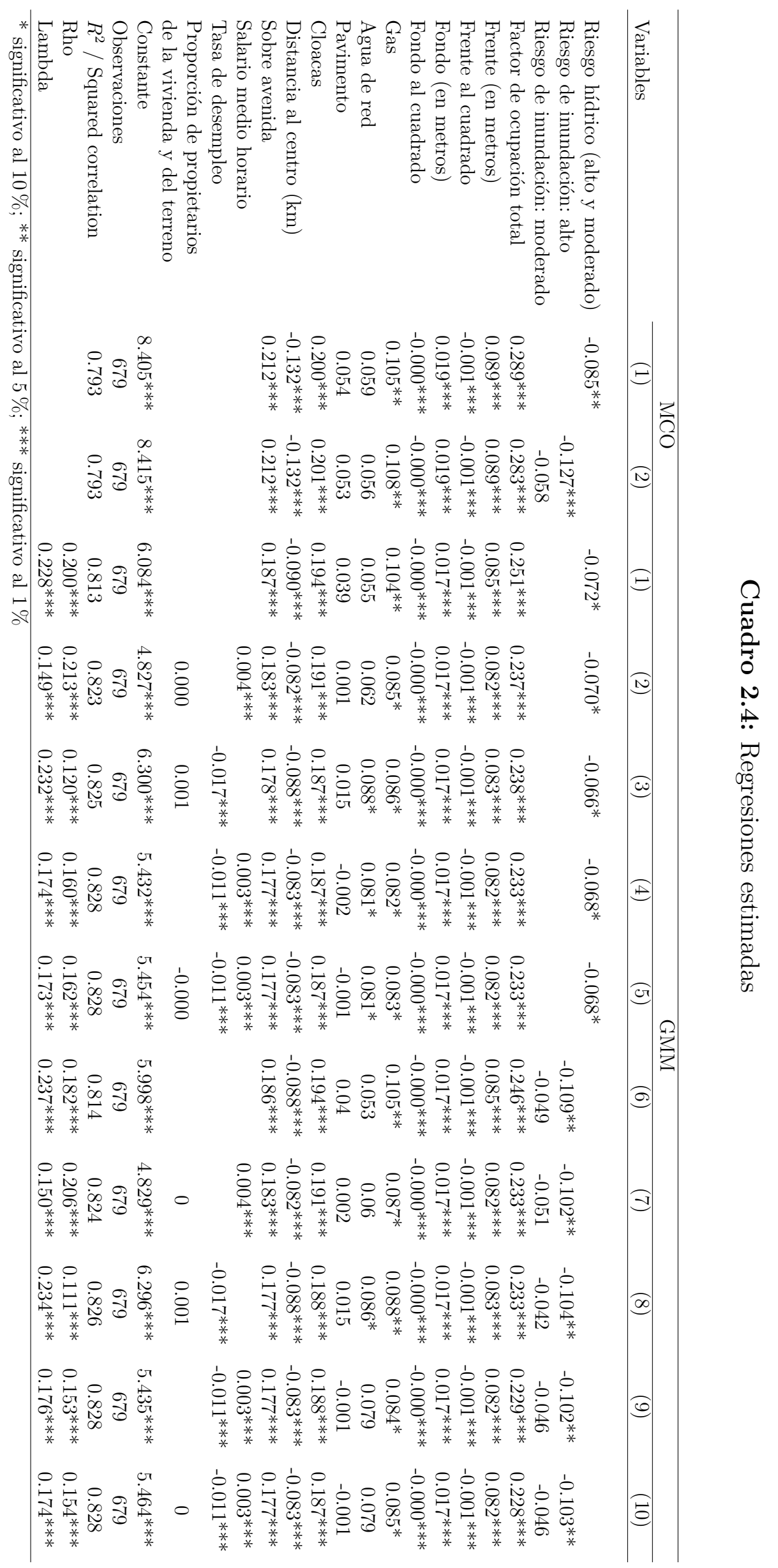


Cuadro 2.5: Efectos marginales de las variables de riesgo de inundación

\begin{tabular}{|c|c|c|c|c|c|}
\hline \multicolumn{6}{|l|}{ A. Riesgo hídrico } \\
\hline Modelo & $(1)$ & $(2)$ & $(3)$ & $(4)$ & $(5)$ \\
\hline Directo efecto & $-0.073^{*}$ & $-0.071^{*}$ & $-0.066^{*}$ & $-0.068^{*}$ & $-0.068^{*}$ \\
\hline Indirecto efecto & $-0.017^{*}$ & $-0.018^{*}$ & $-0.009^{*}$ & $-0.013^{*}$ & $-0.013^{*}$ \\
\hline Total efecto & $-0.09^{*}$ & $-0.089^{*}$ & $-0.075^{*}$ & $-0.081^{*}$ & $-0.081^{*}$ \\
\hline \multicolumn{6}{|l|}{ B. Riesgo alto } \\
\hline Modelo & (6) & $(7)$ & $(8)$ & (9) & $(10)$ \\
\hline Directo efecto & $-0.11^{* *}$ & $-0.103^{* *}$ & $-0.104^{* *}$ & $-0.103^{* *}$ & $-0.104^{* *}$ \\
\hline Indirecto efecto & $-0.023^{* *}$ & $-0.025^{* *}$ & $-0.013^{* *}$ & $-0.018^{* *}$ & $-0.018 * *$ \\
\hline Total efecto & $-0.133^{* *}$ & $-0.128^{* *}$ & $-0.117^{* *}$ & $-0.12^{* *}$ & $-0.122^{* *}$ \\
\hline \multicolumn{6}{|l|}{ C. Riesgo Medio } \\
\hline Modelo & $(6)$ & $(7)$ & $(8)$ & $(9)$ & $(10)$ \\
\hline Directo efecto & -0.049 & -0.052 & -0.042 & -0.046 & -0.046 \\
\hline Indirecto efecto & -0.012 & -0.013 & -0.006 & -0.008 & -0.009 \\
\hline Total efecto & -0.061 & -0.065 & -0.048 & -0.055 & -0.055 \\
\hline
\end{tabular}





\section{Valuación de la contaminación acústica en La Plata}

\section{Introducción}

Muchas ciudades se ven afectadas por los altos niveles de contaminación del aire y el ruido derivados fundamentalmente del tráfico, de la actividad industrial y de las operaciones relacionadas con la construcción. De hecho, la contaminación atmosférica y acústica es uno de los principales problemas de los individuos que habitan las ciudades. En este sentido, el ruido, entendido como un factor de estrés ambiental, puede producir trastornos del sueño y del aprendizaje, de la memoria, de la motivación, en la resolución de problemas y en el incremento de la irritabilidad y de la agresividad. Incluso, cuando la persona consigue afrontar con éxito su respuesta frente a la exposición al ruido ésta puede generar efectos secundarios con consecuencias negativas en la salud y, de esta manera, interferir con el bienestar del individuo.

Los datos de la Organización Mundial de Salud muestran, por un lado, que en la parte occidental de Europa se pierden cada año al menos un millón de años de vida saludable ${ }^{1}$ debido a los ruidos relacionados con el tráfico. Por otro lado, si bien varios países desarrollados han implementado políticas de reducción del ruido en las últimas décadas, se ha calculado que más del $20 \%$ de la Unión Europea (cerca de 80 millones de personas) está expuesta al ruido en niveles que se consideran por encima de los límites aceptables y que provocan molestias, perturbación del sueño y posibles efectos adversos sobre la salud. Adicionalmente, otros 170 millones viven en lo que se conoce como zonas grises, donde los niveles de ruido causan serias molestias durante el día (WHO, 2011). Lamentablemente, no se cuentan con estudios similares para Argentina, como así tampoco para Latinoamérica.

Una valoración precisa de los beneficios asociados con una reducción de los niveles de contaminación acústica es especialmente relevante en el diseño de políticas ambientales, ya que determina si los beneficios de las políticas diseñadas exceden los costos asociados con

\footnotetext{
${ }^{1}$ La Organización Mundial de la Salud, WHO (2011), con supuestos conservadores aplicados a los métodos de cálculo, estiman que los años de vida perdidos ajustados por discapacidad (DAYLs) como consecuencia del ruido ambiental son 61000 años para la enfermedad isquémica del corazón, 45000 años por el deterioro cognitivo de los niños, 903000 años por los trastornos del sueño, 22000 años por el zumbido y 587000 años por las molestias en los Estados miembros de la Unión europea y otros países de Europa occidental.
} 
su implementación. Es importante, entonces, encontrar la forma más adecuada para estimar dichos beneficios y determinar la precisión de los valores estimados. Es esencial comprender cómo estas estimaciones son sensibles a los métodos utilizados, para conocer la fiabilidad de las estimaciones de los beneficios que están siendo utilizadas para la toma de decisiones políticas.

La motivación de este trabajo es contribuir a mejorar la medición de los beneficios asociados a los cambios en los niveles de contaminación acústica. El objetivo de este trabajo consiste en obtener una valoración precisa de la disposición marginal a pagar de los individuos por reducir la contaminación acústica. Los resultados obtenidos ponen de relieve la importancia de dicha reducción: un comprador está dispuesto a pagar alrededor de un 1,9\% menos por cada decibel.

El capitulo está organizado de la siguiente manera: en la primera sección se presenta la revisión de la literatura y en la segunda sección describen los datos utilizados. En la tercera sección se define la estrategia empírica y luego se presentan los resultados obtenidos, mientras que en la quinta sección se realiza un análisis de sensibilidad de los resultados obtenidos. Finalmente, se presentan las conclusiones.

\subsection{Revisión de la literatura}

El enfoque hedónico desarrollado por Rosen (1974) se ha convertido en una metodología establecida en la economía ambiental. Luego de los estudios clásicos sobre contaminación de Ridker \& Henning (1967) y Harrison \& Rubinfeld (1978), se ha generado una voluminosa literatura que trata los aspectos teóricos, metodológicos y empíricos de la economía ambiental. En lo que se relaciona con el valor económico de la contaminación acústica, Navrud (2002) es una buena revisión de la literatura.

A diferencia de lo que se pretende analizar en este trabajo, la mayoría de los estudios anteriores analizan el impacto del ruido a través de la distancia a una fuente importante de contaminación acústica, como son las rutas o las avenidas con mucho tráfico, las estaciones de trenes o los aeropuertos. Dadas las grandes diferencias en los enfoques, en los contextos, en los datos, en las medidas de ruido, en el periodo de tiempo analizado, entre otras, los resultados de los estudios existentes muestran una amplia variedad de estimaciones sobre los impactos económicos del ruido. Las técnicas de meta-análisis son particularmente útiles para la comparación de este tipo de estudios, con el objetivo de identificar posibles factores que expliquen sus diferencias y para evaluar la consistencia de los resultados de investigación. Una revisión de 22 estudios sobre el efecto del ruido generado por el tráfico en las rutas y en las avenidas muestra valoraciones que van desde $0,08 \%$ a $2,22 \%$ por decibel, mientras que estudios que analizan las distancias a los aeropuertos presentan valoraciones que comienzan en 0,06\% y llegan al 2,3\% (Hawkins, 1999; Bateman et al., 2000 y Day, 2001). En otro meta-análisis realizado por Bertrand (1997) citado en Navrud (2002) basado en 16 estimaciones provenientes de 9 estudios internacionales, 
se señala que el impacto promedio es de $0,64 \%$ por decibel. Las variaciones entre los estudios son explicadas, principalmente, por las diferencias en los niveles de ingresos.

Con respecto al impacto del ruido de los aeropuertos, un meta-análisis realizado por Nelson (2004) sobre 20 estudios realizados en Estados Unidos y en Canadá, indicó que el impacto promedio del ruido es de $0,58 \%$ por cada decibel. El estudio muestra que el país y la especificación del modelo tienen efecto sobre el impacto estimado, mientras que las otras variables consideradas (como el tamaño de la muestra y el año del estudio) tienen poco efecto. El efecto de la contaminación acústica de los aeropuertos es de aproximadamente 0,5-0,6\% por decibel en los Estados unidos y 0,8-0,9\% en Canadá.

En lo que se refiere a los trabajos realizados para Argentina, existen dos trabajos que intentan realizar una valuación de la contaminación ambiental. El trabajo realizado por Angeletti (2000) para la ciudad de La Plata encuentra que la contaminación sonora y del aire no juegan un rol fundamental en la determinación del precio de las viviendas. Mientras que el análisis realizado por Conte Grand (2001) para la ciudad de Buenos Aires encuentra una relación negativa en los precios, pero con un valor pequeño y significativo, solamente al nivel de $15 \%$. Un punto que es importante recalcar sobre estos trabajos es que no cuentan con una medida directa del nivel de contaminación. Dada la escasez de información puntual sobre contaminación ambiental, ambos trabajos utilizan como proxy el transporte público de pasajeros, definido como el producto de la cantidad de lineas y su frecuencia. En este trabajo se cuenta con una medida directa del nivel de contaminación acústica con una gran precisión, la cual se describe en la próxima sección.

\subsection{Datos utilizados}

En este capitulo se analiza el efecto que la contaminación acústica tiene sobre los precios de las casas en el casco urbano de la ciudad de La Plata. La ciudad es la capital de la Provincia de Buenos Aires que, según el censo de 2010, tiene una población de 649613 habitantes y 265677 hogares. La Plata es una ciudad planificada; un paradigma de planificación urbana de finales del siglo XIX ${ }^{2}$. El plan original de la ciudad, diseñado por el arquitecto Pedro Benoit, se caracterizaba por una red de líneas rígidas que llevaban a importantes avenidas.

\subsubsection{Mercado de viviendas}

Los datos utilizados en este trabajo incluyen 697 casas en venta durante el año 2010. Los datos provienen de casas en oferta anunciadas por 28 agencias inmobiliarias locales. Estas inmobiliarias, que representan alrededor del $95 \%$ del mercado inmobiliario local, publican sus ofertas en un sistema de información en línea llamado SIOC (Sistema Inmobiliario de Ofertas

${ }^{2}$ La ciudad fue galardonada con dos medallas de oro en las categorías Ciudad del Futuro y Mejor Realización Construida en la Exposición Universal de 1889 en París. 
por Computación). Este sistema proporciona información sobre las propiedades en venta como: la ubicación de la casa, el precio, el tamaño del lote, el número de habitaciones y de baños y la antigüedad de la propiedad. Además de la información obtenida del SIOC, se calculó la distancia hasta el centro comercial de la ciudad ${ }^{3}$. Adicionalmente, se generó una variable dicotómica que indica si la propiedad se encuentra sobre una avenida.

Al igual que en el resto de la Argentina, el mercado inmobiliario de La Plata utiliza con frecuencia el dólar estadounidense como moneda de referencia. Esto se ha convertido en una necesidad en parte debido a la historia de inestabilidad monetaria argentina, que ha empeorado gravemente desde la hiperinflación de 1989-1990. En noviembre de 2011 se comenzó con una serie de restricciones sobre el acceso al mercado cambiario, las cuales complicaron el libre acceso a la moneda extranjera. Dichas restricciones introdujeron distorsiones en el mercado cambiario, lo cual generó un mercado informal de divisas, creó diversas cotizaciones de la moneda y dificultó los precios de referencia del mercado inmobiliario. Luego de dichas restricciones se produjeron fuertes caídas en el volumen de ventas de propiedades inmobiliarias. Desde la introducción de dichas restricciones, y según el Colegio de Escribanos de la Provincia de Buenos Aires, el volumen de escrituras durante el año 2014 representó solo el $60 \%$ del volumen correspondiente al año 2011, pasando de 136214 transacciones a 82348 en 2014. Es por este motivo que no hay datos confiables disponibles sobre la actividad del mercado inmobiliario después del 2011. El trabajo se centrará en la estimación de la disposición por reducciones del nivel de contaminación acústica con datos anteriores a esa fecha.

Es importante destacar que más del $70 \%$ de las viviendas de la muestra se publican en dólares estadounidenses, si bien se puede simplemente convertir los precios en pesos a dólares estadounidenses, o viceversa, para la aplicación empírica, sin embargo, esas casas que figuran en dólares son diferentes a las anunciados en la moneda local ${ }^{4}$. En particular, la calidad de las estructuras de las casas que figuran en dólares tiende a ser mejor, una situación que no se refleja plenamente en las características que se pueden obtener del SIOC. Por ejemplo, dos casas nuevas de muy diferente calidad se publican como en excelente estado; por lo tanto, la variable estado del inmueble (reportada en el SIOC) no logra controlar por todas las diferencias de calidad. Teniendo esto en cuenta, se convirtió el precio de las propiedades a dólares, pero se incluyó una variable dummy que captura la moneda en la que las propiedades fueron listadas originalmente.

El cuadro 3.1 presenta las estadísticas descriptivas de los inmuebles que se utilizarán en este capitulo. Las viviendas tienen un precio promedio de alrededor de 143 mil dólares y cuentan con una superficie cubierta promedio de 199 metros cuadrados. La distancia media al centro comercial es de 8,1 kilómetros. Los inmuebles tienen, en promedio, 3 dormitorios, 2 baños y

\footnotetext{
${ }^{3}$ El centro comercial de la ciudad se estableció en la intersección de las calles 8 y 47. Los cálculos se realizaron con el comando traveltime en Stata, que calcula las distancias de viaje y el tiempo en diversos medios de transporte a través de Google Maps.

${ }^{4}$ Ver cuadro 3.2 en el apéndice.
} 
una antigüedad de poco más de 35 años. El nivel de ruido promedio es de 71 decibeles. A su vez, el $18 \%$ de las casas se encuentra ubicada sobre una avenida y el $77 \%$ tiene cochera. La distribución espacial de los datos utilizados se muestra en la figura 3.1.

\subsubsection{Contaminación acústica}

El mapa de ruido de la ciudad de La Plata fue realizado por el Laboratorio de Acústica y Luminotecnia perteneciente a la Comisión de Investigaciones Científicas de la Provincia de Buenos Aires (LAL-CIC-PBA). Para la realización del mapa se utilizaron 146 puntos de medición ubicados en esquinas, que conformaron una cuadrícula que logró cubrir el casco urbano de la ciudad, con una resolución de 1,5 cuadras $^{5}$. Las mediciones se realizaron entre agosto y noviembre de 2013. En cada esquina se montaron sonómetros, elevados a un metro y medio del suelo mediante trípodes. El tiempo de toma de la muestra fue de 15 minutos consecutivos en las horas pico de lunes a viernes (excluyendo vacaciones y feriados) entre las 11.30 y las 13.30, siempre y cuando las condiciones meteorológicas fueran propicias: ausencia de lluvias y vientos por debajo de los 5 metros por segundo, que equivalen apenas a una brisa suave.

Dado que el sonido no permanece constante a lo largo del tiempo, para valorar lo que ha ocurrido acústicamente a lo largo de un punto del espacio se usan diferentes índices o niveles de valoración. La medida que se utiliza en este trabajo, provista por LAL-CIC-PBA, es el nivel sonoro continuo equivalente (LEQ), que se define como el nivel de un sonido constante en todo el período ${ }^{6}$.

El análisis empírico se basa en ofertas de casas en venta; en consecuencia, existe un pequeño desajuste entre el soporte espacial de la contaminación acústica recogida mediante un conjunto finito de estaciones de monitoreo y el precio de los inmuebles en venta. Existen varias técnicas para producir superficies mediante la estimación/predicción de valores en los lugares no muestreados sobre la base de mediciones en puntos de muestra (Diem, 2003). Estas técnicas de generación de superficies incluyen ponderación por la inversa de la distancia, kriging (kriging ordinario, kriging universal y co-kriging) y por regresión ${ }^{7}$. Los valores de la contaminación acústica reportados por las estaciones de monitoreo se interpolan utilizando kriging ordinario. Es importante tener en cuenta que, dada la distribución espacial de los puntos de medición, la elección del método de interpolación no genera grandes cambios en los resultados. La Figura 3.3 muestra el resultado de los valores de ruido interpolados, el color más oscuro representa los

\footnotetext{
${ }^{5}$ En la figura 3.2 se muestra la distribución de los puntos de medición

${ }^{6} \mathrm{El}$ nivel sonoro continuo equivalente se puede definir como aquel nivel sonoro continuo que proporciona la misma cantidad de energía acústica como el sonido ponderado (A) durante un periodo de tiempo T.

$L_{A E Q, T}=10 . \log \frac{1}{T} \int_{0}^{T}\left(P a(t) / P_{0}\right)^{2} d t$

Donde: T es el tiempo de medición,

$P_{a}(t)$ es el valor instantáneo de la presión sonora y

$P_{0}$ es la presión sonora de referencia $\left(20 \mu P_{a}\right)$

${ }^{7}$ Para mas detalles sobre métodos de interpolación ver Anselin \& Le Gallo (2006).
} 
niveles de ruido más altos. Dicho mapa muestra una cobertura inmejorable de la distribución espacial de la contaminación acústica. El análisis del mapa de ruido sugiere que las avenidas de circunvalación, particularmente 32 entre 1 y 13, 72 entre 1 y 19, y 31 entre 42 y 60, están entre los ejes más ruidosos de la ciudad. También, la avenida 13 entre 32 y plaza Paso; la plaza San Martín e inmediaciones, particularmente hacia plaza Rocha; y polos de concentración urbana como la Torre I de plaza Moreno, la Estación de Trenes, y las escuelas 42 (63 y 22) y 19 (41 y 22). Todos superaron en promedio los 76 decibles (dB), cuando los parámetros recomendados por la Organización Mundial de la Salud, en espacios exteriores, oscilan entre los 60 y los 65 dB.

Si bien existe un desfasaje entre los datos de los inmuebles en venta y los datos sobre el nivel de ruido, no ha habido grandes modificaciones en los principales determinantes de la distribución del ruido. Las rutas del transporte urbano de pasajeros, las zonas peatonales y los principales accesos a la ciudad siguen siendo los mismos. Los resultados obtenidos deben interpretarse como un límite inferior, ya que los individuos pueden haber actualizado sus valoraciones sobre la contaminación acústica desde el 2010 a la fecha. Lamentablemente, no se cuenta con información confiable disponible para el mercado inmobiliario para periodos posteriores al analizado.

\subsection{Estrategia empírica}

La estimación del efecto que tiene la contaminación acústica sobre los valores inmobiliarios se realiza mediante el enfoque de precios hedónicos espaciales descripto en el capítulo metodológico. Es importante remarcar que el modelo hedónico asume que los beneficios derivados de una reducción en el nivel de contaminación acústica se reflejará en el precio que los individuos pagan por la propiedad que elijen, y que todos los compradores y vendedores tienen información perfecta sobre cada atributo de la vivienda. En otras palabras, el fundamento de este enfoque es que, ceteris paribus, las casas ubicadas en zonas con menor contaminación acústica tendrán este beneficio capitalizado en el precio, que debe reflejarse en valores inmobiliarios más altos.

La estrategia empírica comienza con la estimación por MCO de un modelo hedónico básico que relaciona el precio de un inmueble con las características de la propiedad, con la calidad ambiental y con las características socioeconómicas del barrio. Formalmente, se estima el siguiente modelo:

$$
P=X \beta+R \gamma+\mu
$$

donde $P$ es un vector de $N$ por 1 que contiene los precios de las propiedades, $X$ es una matriz de $N$ por $K$ de variables independientes como el tamaño del lote, el número de habitaciones y de baños y la antigüedad de la propiedad. Además, se incluye la distancia hasta el centro 
comercial, si se encuentra ubicada sobre una avenida, si fue publicada en dólares, junto con las características socioeconómicas de la zona. $R$ es una matriz de $N$ por $S$ variables indicadoras del nivel de contaminación acústica y $\mu$ es el término de error.

Una vez obtenidos los errores de la estimación por MCO, se realizan los test de Lagrange propuestos por Anselin (2005) para detectar la existencia de interacciones espaciales. Los resultados de los test obtenidos en el cuadro 3.3 muestran que el multiplicador de Lagrange robusto $L M_{\text {error }}$ es el único significativo y permite rechazar la hipótesis nula de no autocorrelación espacial en favor de la especificación del modelo de error espacial (SEM).

El modelo SEM posee la siguiente especificación:

$$
\begin{aligned}
& P=X \beta+R \gamma+\mu, \\
& \mu=\lambda W \mu+\varepsilon,
\end{aligned}
$$

donde $P, X$, y $R$ son las matrices definidas anteriormente y $\lambda$ es el coeficiente en la estructura autorregresiva espacial de la perturbación $\mu$. Las innovaciones $\varepsilon$ se asumen independientes e idénticamente distribuidas (i.d.d.) o independientes pero heteroscedásticas, donde la heteroscedasticidad es de una forma desconocida. La matriz $W$, de dimensión $\mathrm{N}$ por $\mathrm{N}$, es la matriz de ponderadores espaciales obtenida aplicando el criterio de distancia según la función definida en la sección 1.3 del capítulo 1. El modelo SEM a estimar puede expresarse de la siguiente manera:

$$
P=X \beta+R \gamma+(I-\lambda W)^{-1} \varepsilon
$$

Si el modelo anterior especifica correctamente el proceso generador de datos, la estimación de Mínimos Cuadrados Ordinarios será inapropiada. En estos casos, los errores pueden ser distribuidos en grupos (valores similares en áreas específicas) de una manera tal que den lugar a heterogeneidad espacial continua o discreta (regímenes espaciales) cuando no se especifica adecuadamente el modelo. Por lo tanto, puede conducir a problemas de ineficiencia aunque no de sesgo en los estimadores MCO.

\subsection{Resultados}

Los resultados del análisis sobre cómo la contaminación acústica afecta los precios de las viviendas en la ciudad de La Plata se presenta en el cuadro 3.4, así como también los coeficientes que acompañan las otras características de los inmuebles. 
La variable dependiente es el logaritmo natural del precio de venta de las casas. Las cinco primeras columnas corresponden a estimaciones MCO con diferentes controles, mientras que las columnas restantes muestran las estimaciones de los modelos espaciales realizadas por el Método de Momentos Generalizados (GMM).

La ubicación en una zona con un nivel de ruido mayor afecta negativamente el precio, las estimaciones de MCO (columna 1 a 5) muestran un descuento estadísticamente significativo en el precio de alrededor del 2,1\% por ciento por cada decibel. Por otro lado, las estimaciones de los modelos espaciales también sugieren un descuento en el precio, levemente menor, cercano al 1,9\% por cada decibel. Los descuentos estimados se encuentran en línea con los valores encontrados en la literatura. Es importante notar que el coeficiente estimado podría ser una sobrestimación del efecto del ruido, debido a que la variable de ruido también captura el efecto del tráfico, de la congestión y de la escasez de espacio para estacionar en la zona. Otra característica que se debe tener en cuenta es que estas cifras representan el valor capitalizado de los beneficios de la reducción de la contaminación acústica.

Es necesario tener en cuenta que esta es una medida de cambio marginal y que no debería esperarse que se mantenga para cambios no marginales de la contaminación acústica. Un cambio no marginal probablemente cambiaría la voluntad de los individuos a pagar por una reducción en la contaminación acústica y, posiblemente, también induzca a un nuevo equilibrio de precios de las viviendas que tendría que ser recalculado (Kim et al., 2003).

El valor del coeficiente $\lambda$ que mide la estructura autorregresiva espacial de la perturbación tiene un valor significativo de alrededor de 0,6. El resto de las características de los inmuebles tiene el efecto esperado, los precios de las casas aumentan a medida que se incrementa la superficie del lote y la cantidad de metros cubiertos. Del mismo modo, las casas con más habitaciones, baños, garaje y plantas tienden a tener valoraciones más altas. La relación entre la antigüedad y el precio parece no ser significativa. Las viviendas ubicadas sobre avenidas no tienen un efecto significativo y las que se encuentran ubicadas sobre zonas inundables tienen un descuento significativo en el precio. En cuanto a las distancias al centro comercial se encuentra un valor positivo y significativo para las casas más cercanas. Por último, las características socioeconómicas de la zona, como el ingreso de los hogares y la tasa de desempleo, potencialmente correlacionadas con los atributos de vecinos, tienen el efecto esperado en los precios inmobiliarios.

\subsection{Sensibilidad de los resultados}

El mapa de ruido con el cual se trabajó en este capítulo fue elaborado por el Laboratorio de Acústica y Luminotecnia de la Comisión de Investigaciones Científicas de la Provincia de Buenos Aires (LAL-CIC-PBA). Es su realización se utilizaron 146 puntos de medición, que 
conformaron una cuadrícula que logró cubrir el área urbana de la ciudad, con una resolución de 1.5 cuadras; es decir, la distancia máxima al punto de medición es de 150 metros. Esta es una de las resoluciones más altas con las cuales se puede trabajar. Sin embargo, no siempre es posible contar con semejante resolución, principalmente debido a los costos relacionados con este tipo de tareas.

En esta sección se intenta analizar cuáles serían los costos, tanto económicos como en términos de precisión de trabajar con un mapa de ruido realizado con una menor cantidad de puntos de medición y, por ende, con menores costos económicos. Esta es una pregunta válida que podrían hacerse los responsables de los organismos públicos al momento de decidir realizar un mapa de ruido en una ciudad determinada.

Para esto se utilizan los mismos datos provistos por LAL-CIC-BA, solo que se seleccionaron algunos de los puntos para la realización de un mapa de ruido alternativo y luego se compararon los resultados obtenidos.

Una alternativa es realizar una distribución aleatoria de los puntos de medición y analizar el mapa de ruido resultante. Sin embargo, no sería razonable que las autoridades realizaran este tipo de acciones. En este sentido, se plantean cuatro escenarios. El primer escenario contiene 73 puntos de medición distribuidos de manera uniforme por el casco urbano de la ciudad, lo que da como resultado una resolución aproximada de 3 cuadras. El mapa 3.4 muestra la distribución de los puntos de medición. Este escenario representaría un 50\% del costo de realización del mapa original, asumiendo un costo constante por cada unidad de medición.

La segunda alternativa contiene 40 puntos de medición distribuidos de manera uniforme por el casco urbano de la ciudad, lo que da como resultado una resolución aproximada de poco más de 5 cuadras. El mapa 3.5 muestra la distribución de los puntos de medición. Este escenario representaría un $34 \%$ del costo de realización del mapa original, asumiendo un costo constante por cada unidad de medición.

El tercer escenario ubica los puntos de medición en los cruces de las avenidas, una actitud que razonablemente realizarían los gobiernos. Este escenario contaría con 43 puntos de medición, lo que da como resultado una resolución aproximada de 5 cuadras y representaría un $29 \%$ del costo de realización del mapa original. El mapa 3.6 muestra la distribución de los puntos de medición bajo este escenario.

En el último escenario se consideran todos los puntos de medición que se ubican sobre las avenidas. Este escenario contaría con 113 puntos de medición, lo que da como resultado una resolución aproximada de 2 cuadras, cuya distribución se muestra en el mapa 3.7 . Este escenario representaría un $77 \%$ del costo de realización del mapa original.

La forma en la que se analizan las divergencias entre las mediciones de ruido de los distintos escenarios consistió en la extracción de más de 5000 puntos aleatoriamente distribuidos en los mapas de ruido y luego se los comparó con los valores del mapa original. 
El primer escenario muestra una subestimación de aproximadamente un -0,30\%, con diferencias que van desde $-7,5 \%$ a $11,10 \%$. El $50 \%$ de las diferencias se encuentra entre $-1,3 \%$ (percentil 25) y $0,5 \%$ (percentil 75), como lo muestra el cuadro 3.5. En el segundo escenario se observa una sobrestimación de aproximadamente un 1,3\%, con valores que oscilan entre -6,5\% y 12,7\%, mientras que el $50 \%$ de las divergencias tienen valores entre $0,1 \%$ y 2,40\%. Mientras que en el tercer escenario se observa una sobrestimación de aproximadamente un $1 \%$, con valores que van desde $-6,6 \%$ a $12,50 \%$, mientras que el $50 \%$ de las divergencias se presentan entre $-0,2 \%$ y $1,9 \%$. Finalmente, en el cuarto escenario se observa una sobrestimación de aproximadamente un $0,6 \%$, con valores que van desde $-7,2 \%$ a 10,3\%. En este caso, el $50 \%$ de las diferencias se encuentra entre $0 \%$ y $0,9 \%$.

Otro tema interesante para analizar es preguntarse cuáles serían los resultados de la disposición marginal a pagar por la reducción de la contaminación acústica si en lugar de utilizar el mapa de ruido de LAL-CIC-BA, se utilizara el mapa realizado en cada escenario. Los cuadros 3.6, $3.7,3.8$ y 3.9 muestran los resultados de las estimaciones realizadas con los mapas de ruido del escenario 1, 2, 3 y 4, respectivamente.

Las diferencias en los coeficientes serían, en promedio, de 0,00012 en el primer escenario, con diferencias que oscilan entre -0,0005 y 0,0006, según los controles que se utilicen en la regresión. Estas diferencias representan divergencias en promedio del $-0,6 \%$, con valores que van desde $-2,9 \%$ hasta $2,6 \%$.

En el segundo escenario, la diferencia en el coeficiente de la regresión sería de aproximadamente 0,00408, con diferencias que oscilan entre 0,003 y 0,0046. Estas divergencias son valores en promedio cercanos al $-20,8 \%$ en el valor del coeficiente, con valores que van desde $-24,7 \%$ hasta $-13,6 \%$.

Las diferencias en los coeficientes serían, en promedio, de 0,0003 en el escenario 3, con diferencias que oscilan entre $-0,0015$ y 0,003 . Estas diferencias representan divergencias en promedio del $1,3 \%$, con valores que van desde $-1,6 \%$ hasta $6,8 \%$.

En el último escenario, la diferencia en el coeficiente de la regresión sería de aproximadamente 0,0007, con diferencias que oscilan entre -0,0018 y 0,0039. Estas divergencias son valores en promedio cercanos al $-3,0 \%$ en el valor del coeficiente, con valores que van desde $-17,6 \%$ hasta $9,3 \%$.

Los resultados muestran que no hay grandes pérdidas de eficiencia, si se realiza un análisis con menor cantidad de puntos de medición, si esa selección de puntos de medición se realiza cuidadosamente. Sin embargo, el resultado depende del objetivo del trabajo. Como era de esperarse, los mayores errores de precisión se encuentran en las zonas que se encuentran más distanciadas de los puntos de medición y, sobre todo, en las zonas entre las avenidas en los últimos escenarios. En el caso de que esas zonas sean las relevantes para el análisis la pérdida de eficiencia sería importante. 


\subsection{Conclusiones}

Muchas ciudades se ven afectadas por los altos niveles de contaminación del aire y de ruido derivados fundamentalmente del tráfico, de la actividad industrial y de las operaciones relacionadas con la construcción. De hecho, la contaminación (atmosférica y acústica) es uno de los principales problemas de los individuos que habitan las ciudades. En este sentido, el ruido, entendido como un factor de estrés ambiental, puede intervenir en trastornos del sueño y del aprendizaje, de la memoria, de la motivación, en la resolución de problemas y en el incremento de la irritabilidad y de la agresividad.

Una valoración precisa de los beneficios asociados con una reducción de los niveles de contaminación acústica es especialmente relevante en el diseño de políticas públicas, ya que determina si los beneficios de las políticas diseñadas exceden los costos asociados con su implementación. En este capítulo se ha estimado la disposición a pagar por reducir la contaminación acústica utilizando modelos hedónicos espaciales y un mapa de ruido con gran resolución para la ciudad de La Plata. Los resultados sugieren que cuando las propiedades se encuentran en zonas más ruidosas sufren una reducción en el precio. Dicho descuento es de aproximadamente un 1,9\% en el precio del inmueble por cada decibel.

Se considera que este estudio tiene importantes implicancias en las decisiones sobre la realización de inversiones en infraestructura, como puede ser la selección de una capa asfáltica menos ruidosa o el rediseño de la red de transporte público. La disponibilidad a pagar estimada debe ser confrontada con los costos esperados de los distintos proyectos de control ambiental con el fin de priorizar las inversiones.

Adicionalmente, el trabajo analiza la sensibilidad de los costos, tanto económicos como en términos de precisión, de trabajar con mapas del ruido realizados con una menor cantidad de puntos de medición. Un tema relevante para los organismos públicos que estén evaluando realizar un mapa de ruido. Los resultados del análisis de sensibilidad muestran que no hay grandes pérdidas de eficiencia si se realiza un análisis con menor cantidad de puntos de medición, siempre y cuando los puntos de medición sean cuidadosamente seleccionados. 


\subsection{Apéndice}

Cuadro 3.1: Estadísticas descriptivas

\begin{tabular}{lccccc}
\hline Variables & Media & Desvío estándar & Mínimo & Máximo & Fuente \\
\hline Precio (USD) & 132,770 & 75,731 & 19,139 & 450,000 & $\mathrm{f} 1$ \\
Ruido (LEQ) & 71 & 1 & 66 & 76 & $\mathrm{f} 2$ \\
Dólar & $72 \%$ & $45 \%$ & $0 \%$ & $100 \%$ & $\mathrm{f} 1$ \\
Superficie del lote & 311 & 225 & 40 & 2,160 & $\mathrm{f} 1$ \\
Superficie cubierta & 198 & 106 & 32 & 800 & $\mathrm{f} 1$ \\
Número de plantas & 1.7 & 0.6 & 1.0 & 4.0 & $\mathrm{f} 1$ \\
Número de dormitorios & 3.0 & 1.1 & 1.0 & 10.0 & $\mathrm{f} 1$ \\
Número de baños & 2.3 & 1.2 & 1 & 5.0 & $\mathrm{f} 1$ \\
Antigüedad & 35.2 & 17.4 & 0 & 100.0 & $\mathrm{f} 1$ \\
Cochera & $77 \%$ & $42 \%$ & $0 \%$ & $100 \%$ & $\mathrm{f} 1$ \\
Calidad 2 & $62 \%$ & $49 \%$ & $0 \%$ & $100 \%$ & $\mathrm{f} 1$ \\
Calidad 3 & $27 \%$ & $44 \%$ & $0 \%$ & $100 \%$ & $\mathrm{f} 1$ \\
Periodo 2 (Mayo) & $35 \%$ & $48 \%$ & $0 \%$ & $100 \%$ & $\mathrm{f} 1$ \\
Periodo 3 (Septiembre) & $30 \%$ & $46 \%$ & $0 \%$ & $100 \%$ & $\mathrm{f} 1$ \\
Distancia al centro (km) & 8.1 & 2.6 & 0.3 & 14.2 & $\mathrm{f} 3$ \\
Tiempo en auto al centro (Minutos) & 3.2 & 1.3 & 0.1 & 6.3 & $\mathrm{f} 3$ \\
Sobre avenida & $18 \%$ & $39 \%$ & $0 \%$ & $100 \%$ & $\mathrm{f} 4$ \\
Salario medio horario & 559 & 38 & 441 & 655 & $\mathrm{f} 5$ \\
Tasa de desempleo & $17 \%$ & $5 \%$ & $4 \%$ & $42 \%$ & $\mathrm{f5}$ \\
Proporción de propietarios & $71 \%$ & $9 \%$ & $40 \%$ & $86 \%$ & $\mathrm{f} 5$ \\
Riesgo de inundación: alto & $8 \%$ & $28 \%$ & $0 \%$ & $100 \%$ & $\mathrm{f} 6$ \\
Riesgo de inundación: moderado & $51 \%$ & $50 \%$ & $0 \%$ & $100 \%$ & $\mathrm{f} 6$ \\
\hline
\end{tabular}

Nota: Los precios de los inmuebles son en dólares estadounidenses. Las fuentes son f1: Sistema Inmobiliario de Ofertas por Computacion (SIOC); f2: Laboratorio de Acústica y Luminotecnia de la Comisión de Investigaciones Científicas de la Provincia de Buenos Aires; f3: SIOC y herramientas geoespaciales; f4: elaboración propia en base a dirección postal; f5: Censo 2001 a nivel de radio censal; f6: Instituto de Geomorfologia y Suelos y herramientas geoespaciales. 
Cuadro 3.2: Estadísticas descriptivas

\begin{tabular}{lccccc}
\hline Variables & Media Dólar & Desvío dólar & Media pesos & Desvío pesos & Diferencia \\
\hline Precio (USD) & 76,814 & 38,720 & 154,661 & 75,440 & $77847^{* * *}$ \\
Ruido (LEQ) & 71 & 2 & 71 & 1 & 0 \\
Superficie del lote & 247 & 133 & 337 & 247 & $90^{* * *}$ \\
Superficie cubierta & 143 & 87 & 219 & 105 & $76^{* * *}$ \\
Número de plantas & 1.5 & 0.6 & 1.7 & 0.6 & $0.28^{* * *}$ \\
Número de dormitorios & 2.6 & 0.8 & 3.2 & 1.1 & $0.59^{* * *}$ \\
Número de baños & 1.7 & 0.9 & 2.5 & 1.2 & $0.81^{* * *}$ \\
Antigüedad & 32.6 & 17.8 & 36.3 & 17.1 & $3.69^{* *}$ \\
Cochera & $65 \%$ & $48 \%$ & $82 \%$ & $39 \%$ & $0.17^{* * *}$ \\
Calidad 2 & $71 \%$ & $46 \%$ & $59 \%$ & $49 \%$ & $-0.12^{* * *}$ \\
Calidad 3 & $14 \%$ & $35 \%$ & $31 \%$ & $46 \%$ & $0.17^{* * *}$ \\
Periodo 2 (Mayo) & $32 \%$ & $47 \%$ & $36 \%$ & $48 \%$ & 0.04 \\
Periodo 3 (Septiembre) & $27 \%$ & $44 \%$ & $31 \%$ & $46 \%$ & 0.04 \\
Distancia al centro (km) & 9.6 & 2.4 & 7.5 & 2.5 & $-2.16^{* * *}$ \\
Sobre avenida & $14 \%$ & $35 \%$ & $20 \%$ & $40 \%$ & $0.06^{*}$ \\
Salario medio horario & 538 & 38 & 567 & 34 & $29.5^{* * *}$ \\
Tasa de desempleo & $19 \%$ & $6 \%$ & $17 \%$ & $5 \%$ & $-0.02^{* * *}$ \\
Proporción de propietarios & $73 \%$ & $8 \%$ & $71 \%$ & $10 \%$ & $-0.02^{* * *}$ \\
Riesgo de inundación: alto & $-6 \%$ & $24 \%$ & $-9 \%$ & $29 \%$ & -0.03 \\
Riesgo de inundación: moderado & $-42 \%$ & $50 \%$ & $-54 \%$ & $50 \%$ & $-0.12^{* * *}$
\end{tabular}

* significativo al $10 \%$; ${ }^{* *}$ significativo al $5 \%$; *** significativo al $1 \%$

Cuadro 3.3: Diagnostico espacial

\begin{tabular}{|c|c|c|c|c|}
\hline \multirow{2}{*}{\multicolumn{2}{|c|}{ Modelo }} & \multicolumn{3}{|c|}{ Estadístico } \\
\hline & & I de Moran & Multiplicador de Lagrange & Multiplicador de Lagrange Robusto \\
\hline \multirow[t]{2}{*}{1} & Spatial error & $12.632^{* * *}$ & $147.441^{* * *}$ & $83.494^{* * *}$ \\
\hline & Spatial lag & & $64.177^{* * *}$ & 0.23 \\
\hline \multirow[t]{2}{*}{2} & Spatial error & $12.467 * * *$ & $141.846^{* * *}$ & $83.494^{* * *}$ \\
\hline & Spatial lag & & $56.316^{* * *}$ & 0.23 \\
\hline \multirow[t]{2}{*}{3} & Spatial error & $12.252^{* * *}$ & $141.846^{* * *}$ & $85.538 * * *$ \\
\hline & Spatial lag & & $56.316^{* * *}$ & 0.009 \\
\hline \multirow[t]{2}{*}{4} & Spatial error & $12.357^{* * *}$ & $136.747^{* * *}$ & $84.606^{* * *}$ \\
\hline & Spatial lag & & $54.898^{* * *}$ & 0 \\
\hline \multirow[t]{2}{*}{5} & Spatial error & $12.28^{* * *}$ & $136.746^{* * *}$ & $84.15^{* * *}$ \\
\hline & Spatial lag & & $52.635^{* * *}$ & 0.039 \\
\hline
\end{tabular}

* significativo al $10 \%$; ${ }^{* *}$ significativo al $5 \%$; *** significativo al $1 \%$

El modelo 1 incluye controles por riesgo de inundación alto y moderado. El modelo 2 incluye controles por riesgo de inundación alto y moderado, salario medio y proporción de propietarios. El modelo 3 incluye controles por riesgo de inundación alto y moderado, tasa de desocupación y proporción de propietarios. El modelo 4 incluye controles por riesgo de inundación alto y moderado, salario medio y tasa de desocupación. El modelo 5 incluye controles por riesgo de inundación alto y moderado, salario medio, tasa de desocupación y proporción de propietarios. 
Figura 3.1: Distribución espacial de las viviendas

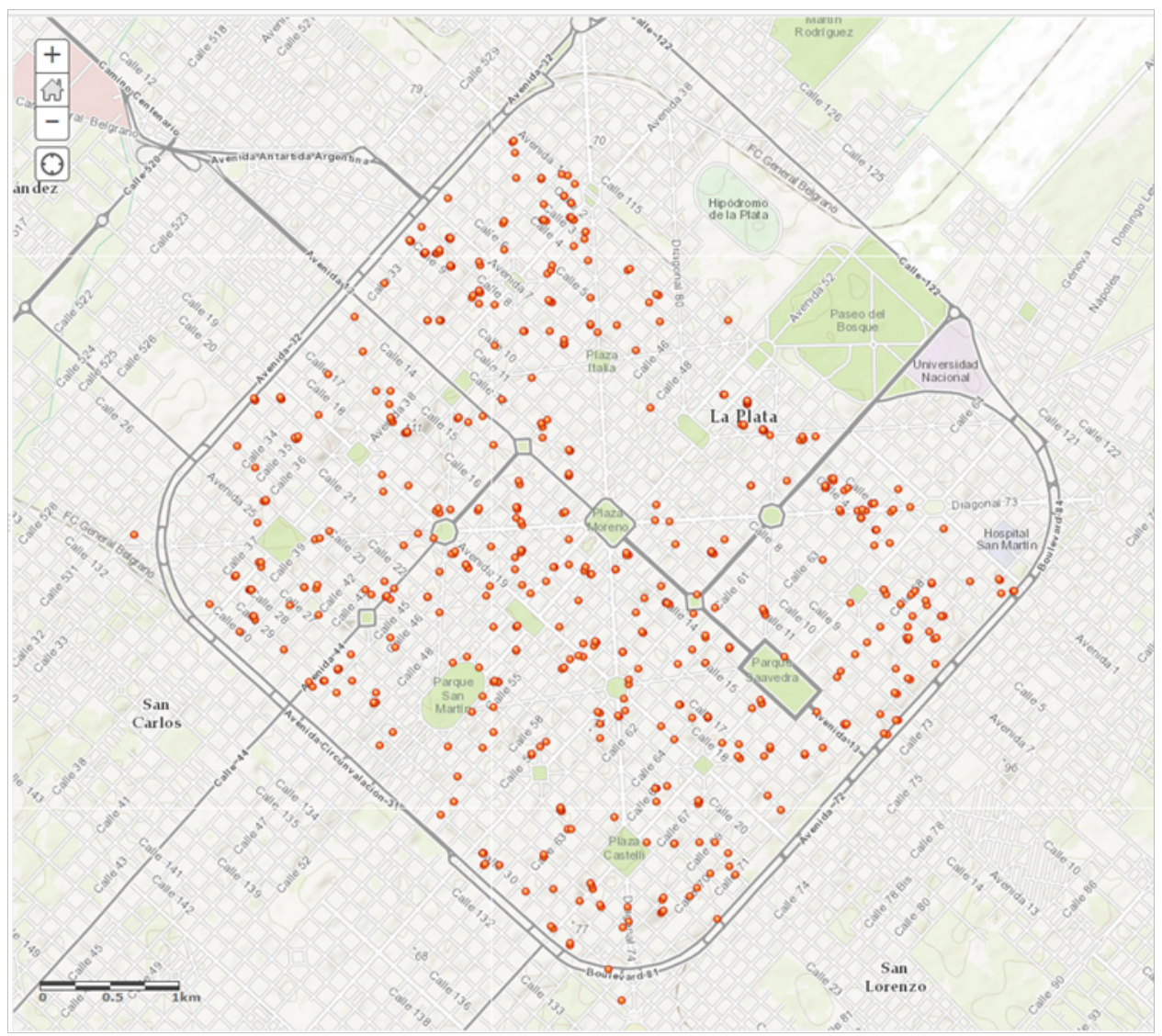

Figura 3.2: Distribución espacial de los puntos de medición

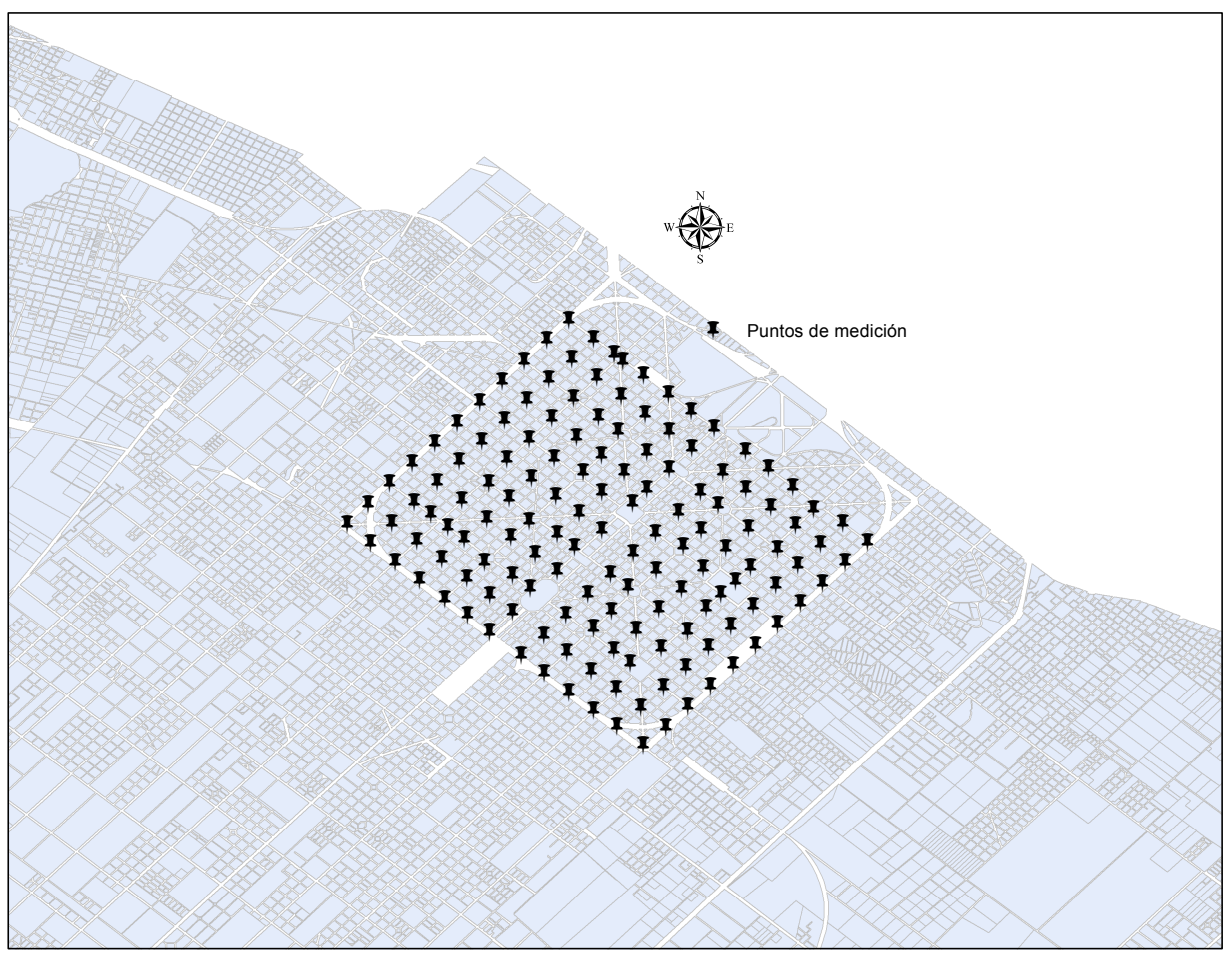


Figura 3.3: Interpolación de la contaminación acústica

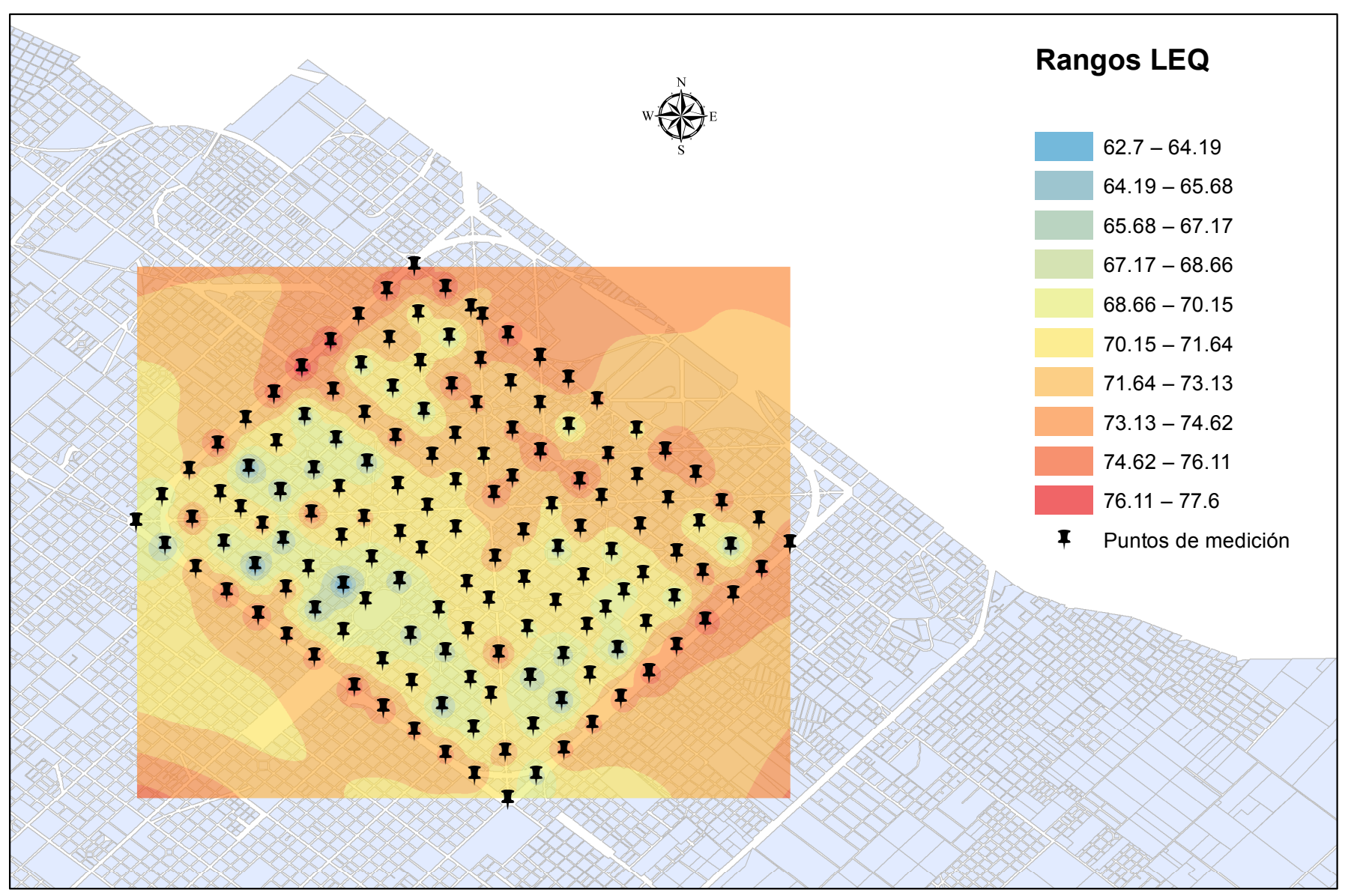


Cuadro 3.4: Regresiones estimadas

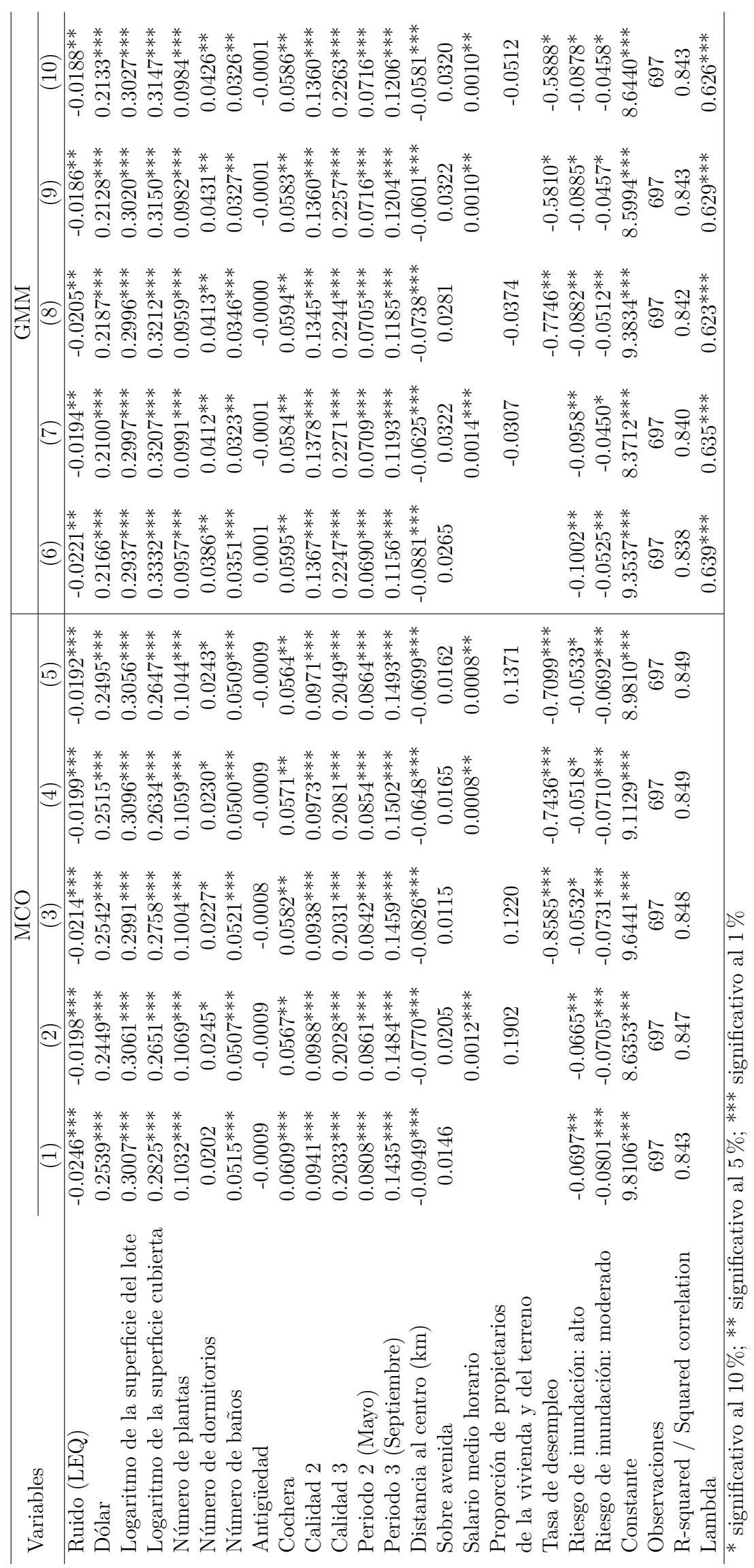


Cuadro 3.5: Estadísticas descriptivas y diferncias con el Mapa de LAL-CIC-BA

\begin{tabular}{lcccc}
\hline \multirow{2}{*}{ Estadísticos } & \multicolumn{4}{c}{ Escenario } \\
\cline { 2 - 5 } & 1 & 2 & 3 & 4 \\
\hline Media & $-0.30 \%$ & $1.30 \%$ & $1.00 \%$ & $0.60 \%$ \\
Desvío estándar & $1.70 \%$ & $2.10 \%$ & $1.90 \%$ & $1.40 \%$ \\
Mínimo & $-7.50 \%$ & $-6.50 \%$ & $-6.60 \%$ & $-7.20 \%$ \\
Percentil 1 & $-4.90 \%$ & $-3.20 \%$ & $-3.30 \%$ & $-2.70 \%$ \\
Percentil 5 & $-3.00 \%$ & $-1.90 \%$ & $-1.90 \%$ & $-0.70 \%$ \\
Percentil 10 & $-2.20 \%$ & $-1.20 \%$ & $-1.40 \%$ & $-0.30 \%$ \\
Percentil 25 & $-1.30 \%$ & $0.10 \%$ & $-0.20 \%$ & $0.00 \%$ \\
Mediana & $-0.20 \%$ & $1.30 \%$ & $1.00 \%$ & $0.40 \%$ \\
Percentil 75 & $0.50 \%$ & $2.40 \%$ & $1.90 \%$ & $0.90 \%$ \\
Percentil 90 & $1.30 \%$ & $3.80 \%$ & $3.10 \%$ & $1.90 \%$ \\
Percentil 95 & $2.00 \%$ & $4.70 \%$ & $4.20 \%$ & $3.00 \%$ \\
Percentil 99 & $4.50 \%$ & $7.90 \%$ & $7.20 \%$ & $6.40 \%$ \\
Máximo & $11.10 \%$ & $12.70 \%$ & $12.50 \%$ & $10.30 \%$ \\
Puntos de observación & 73 & 40 & 43 & 113 \\
Costo relativo & $50.00 \%$ & $27.40 \%$ & $29.50 \%$ & $77.40 \%$ \\
\hline
\end{tabular}


Cuadro 3.6: Regresiones estimadas (escenario 1)

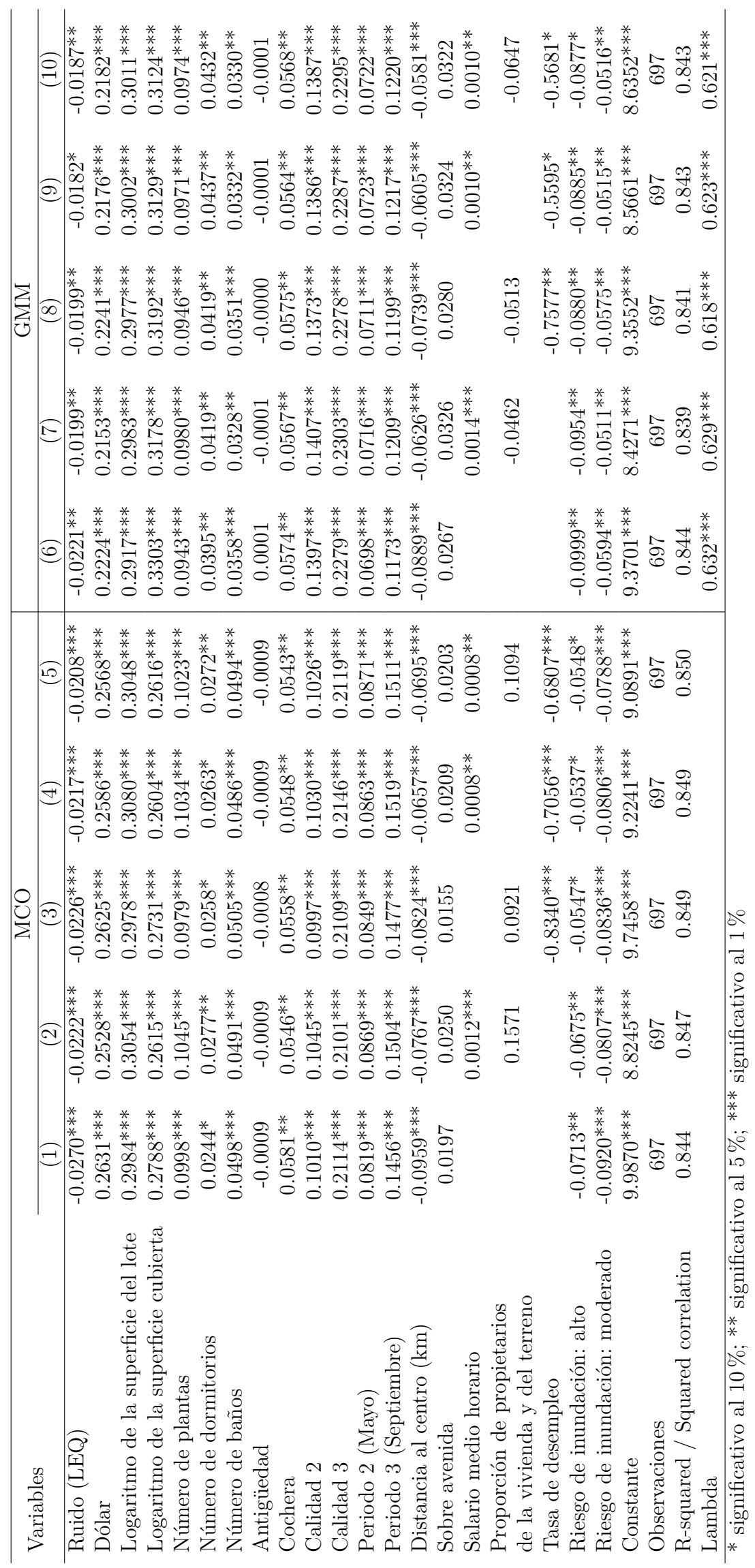


Cuadro 3.7: Regresiones estimadas (escenario 2)

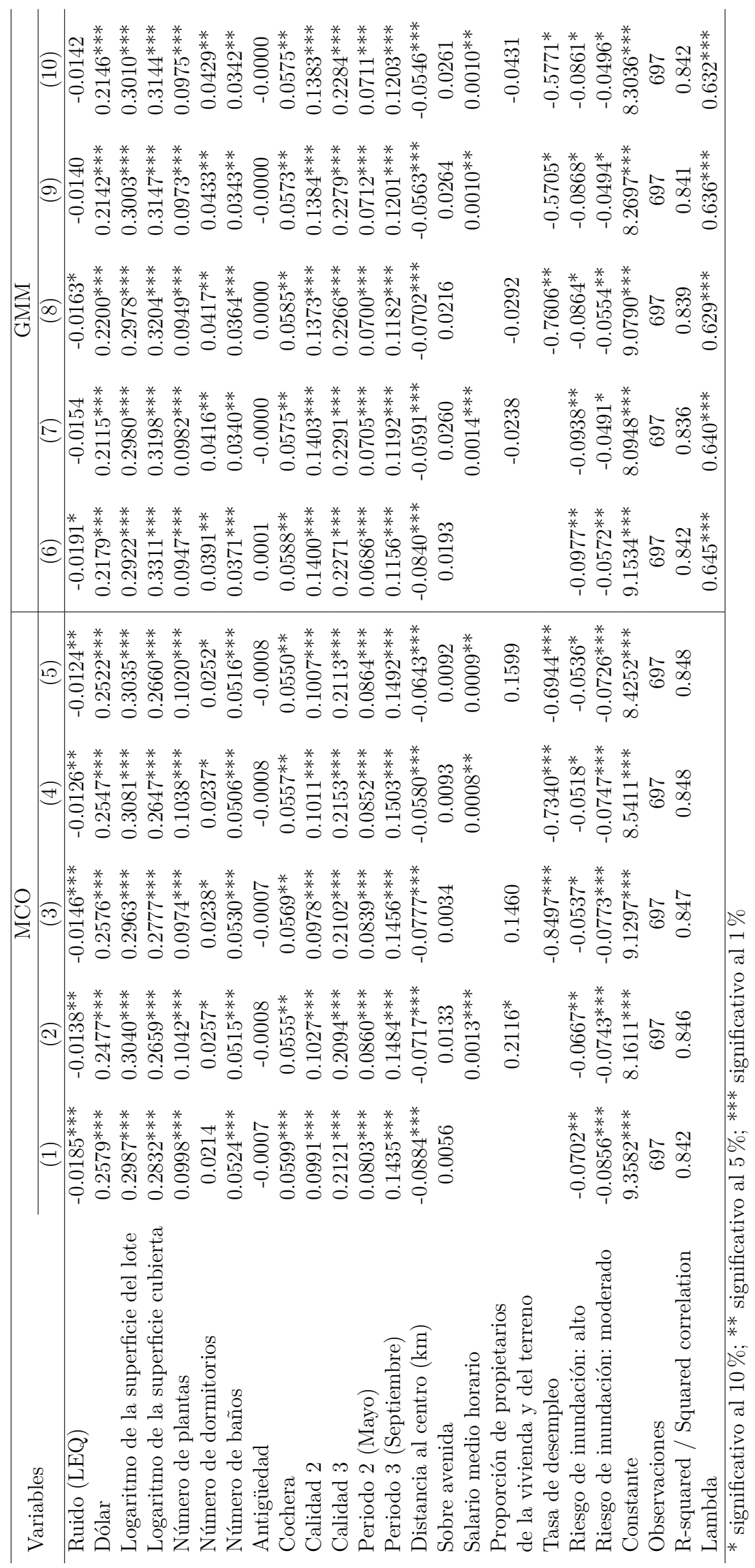


Cuadro 3.8: Regresiones estimadas (escenario 3)

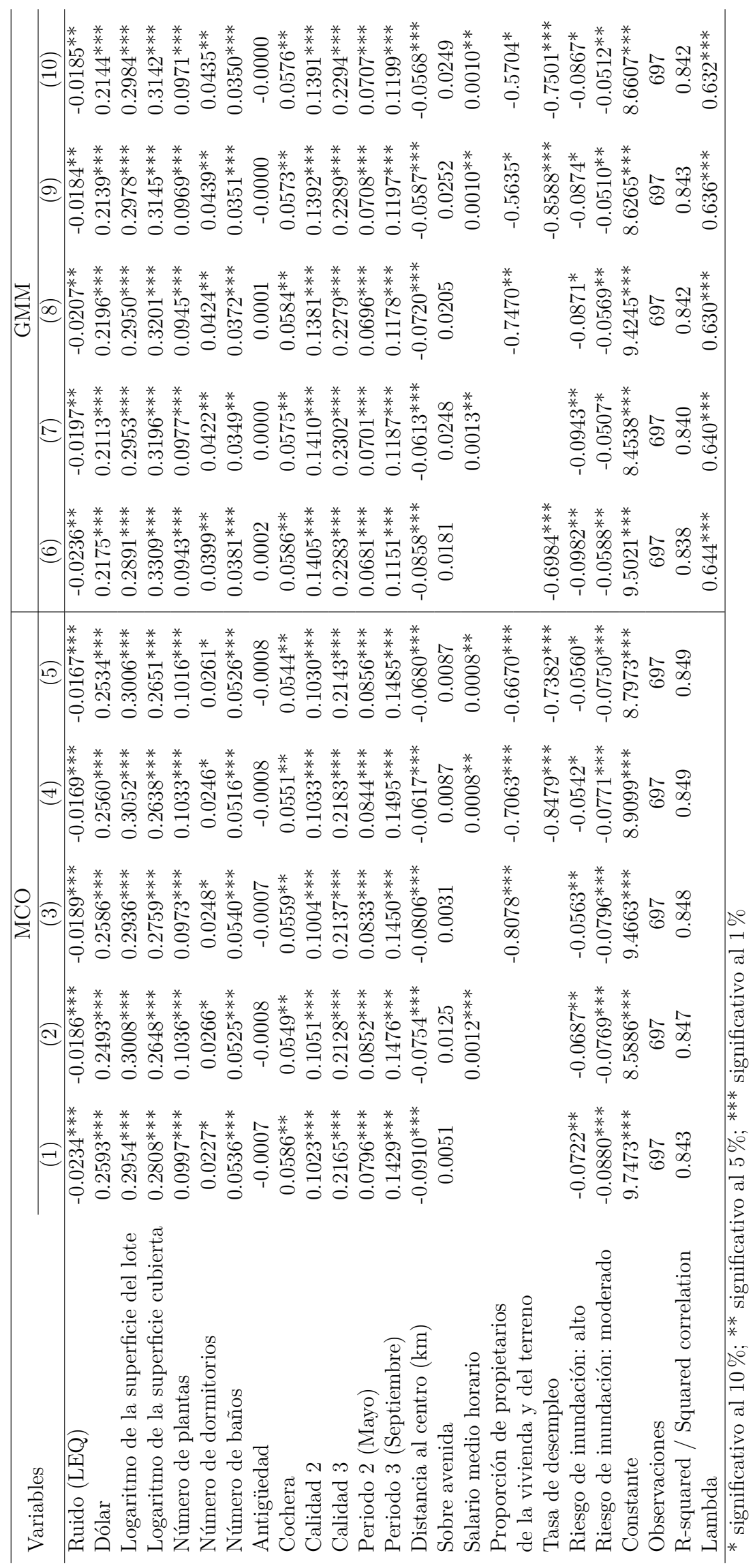


Cuadro 3.9: Regresiones estimadas (escenario 4)

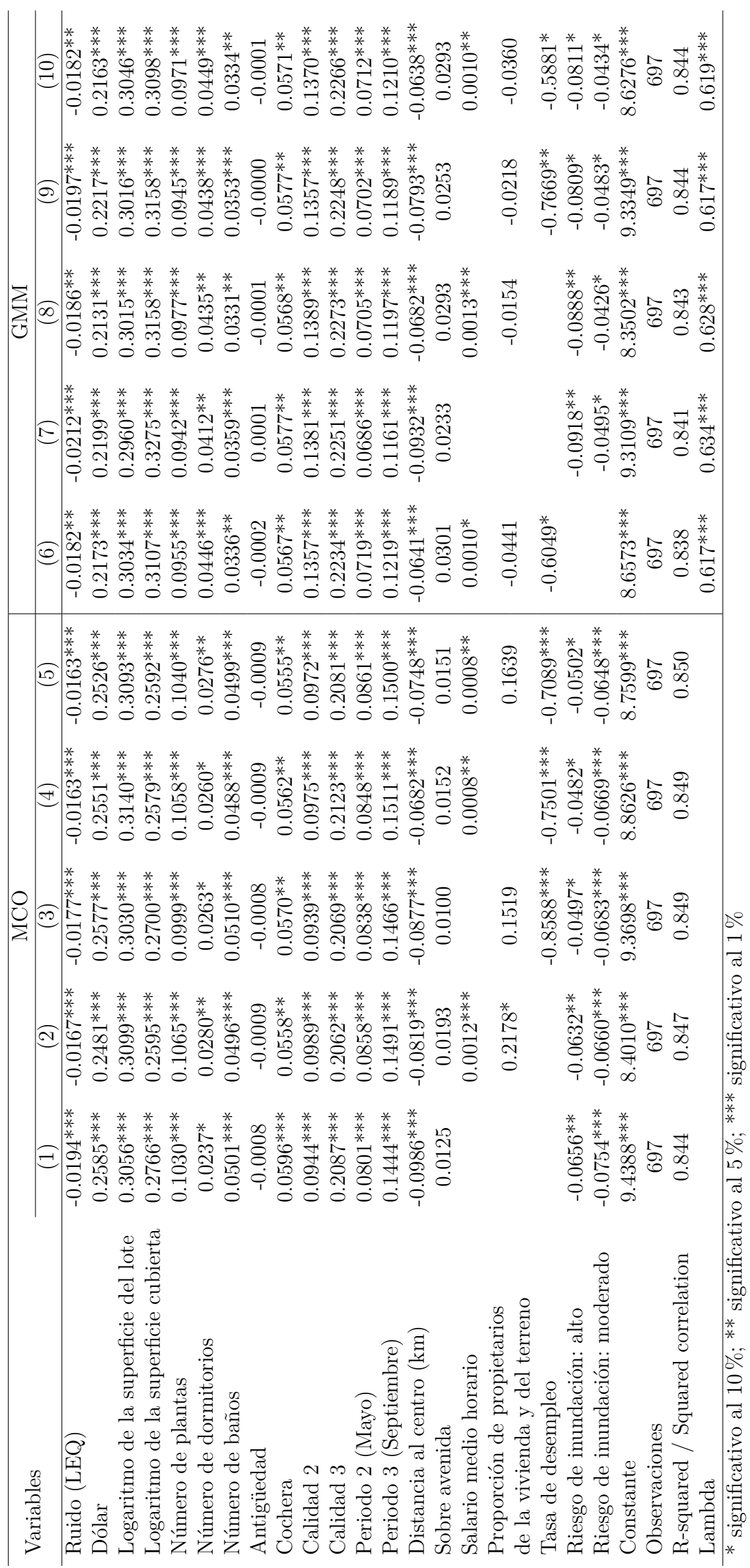


Figura 3.4: Mapa del ruido, escenario 1

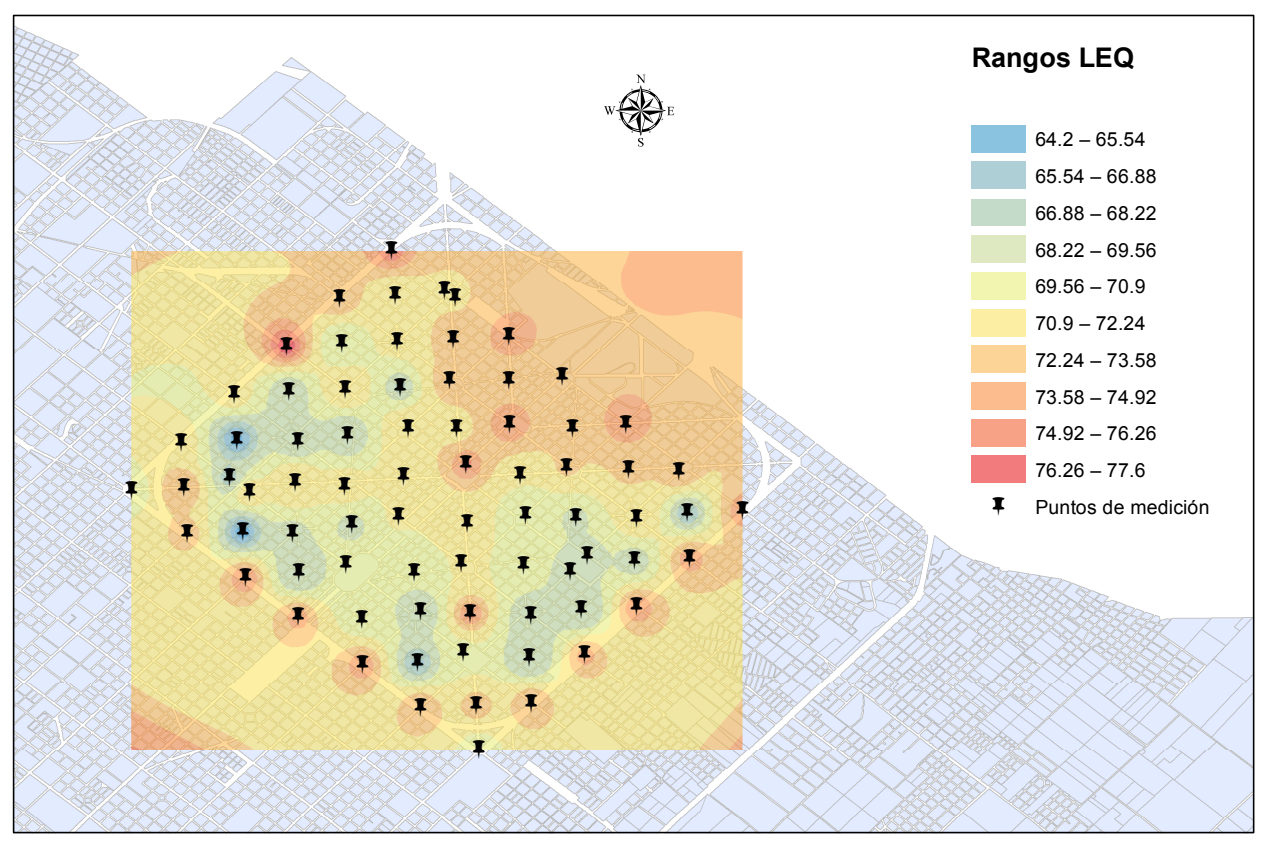

Figura 3.5: Mapa del ruido, escenario 2

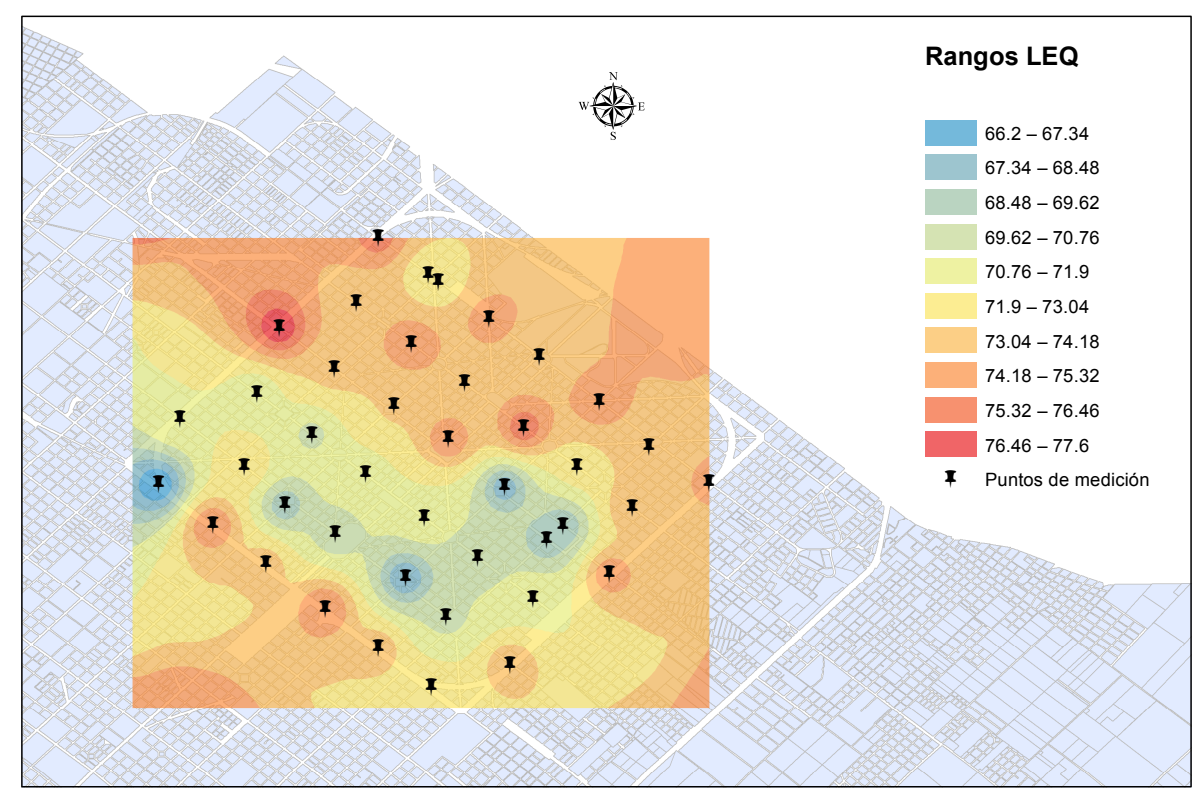


Figura 3.6: Mapa del ruido, escenario 3

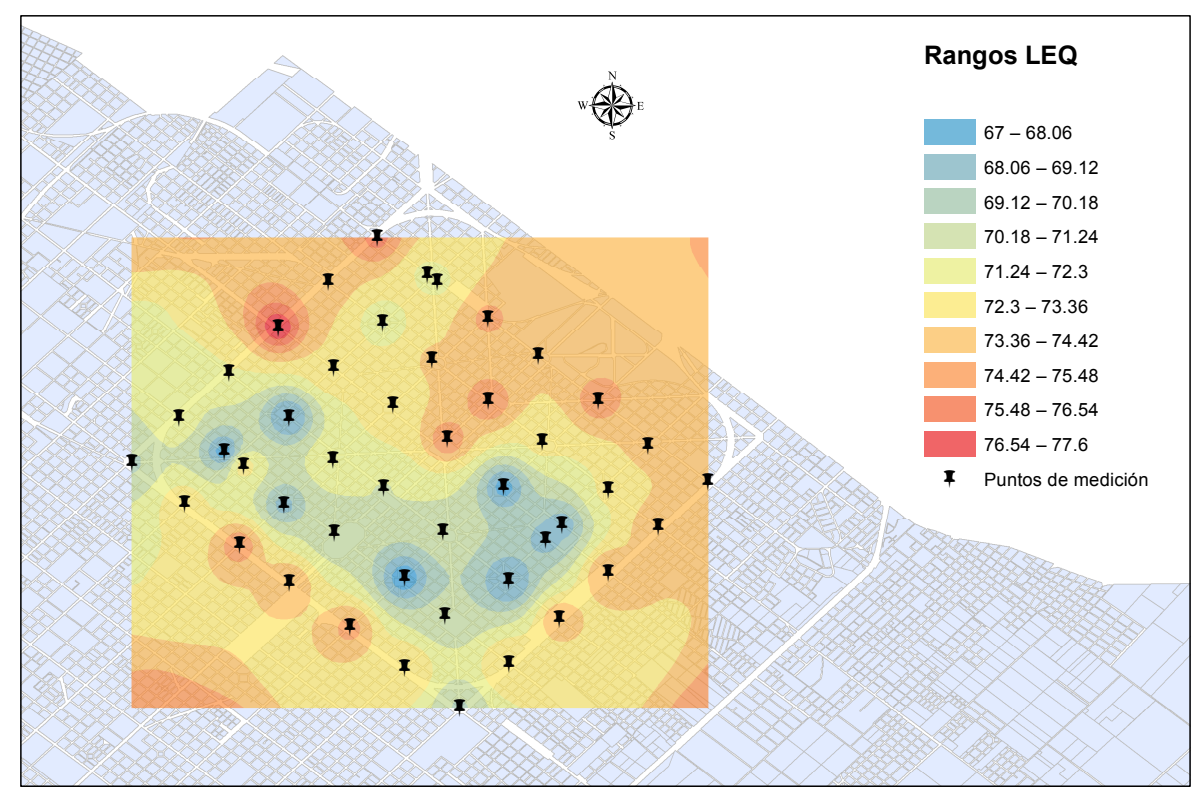

Figura 3.7: Mapa del ruido, escenario 4

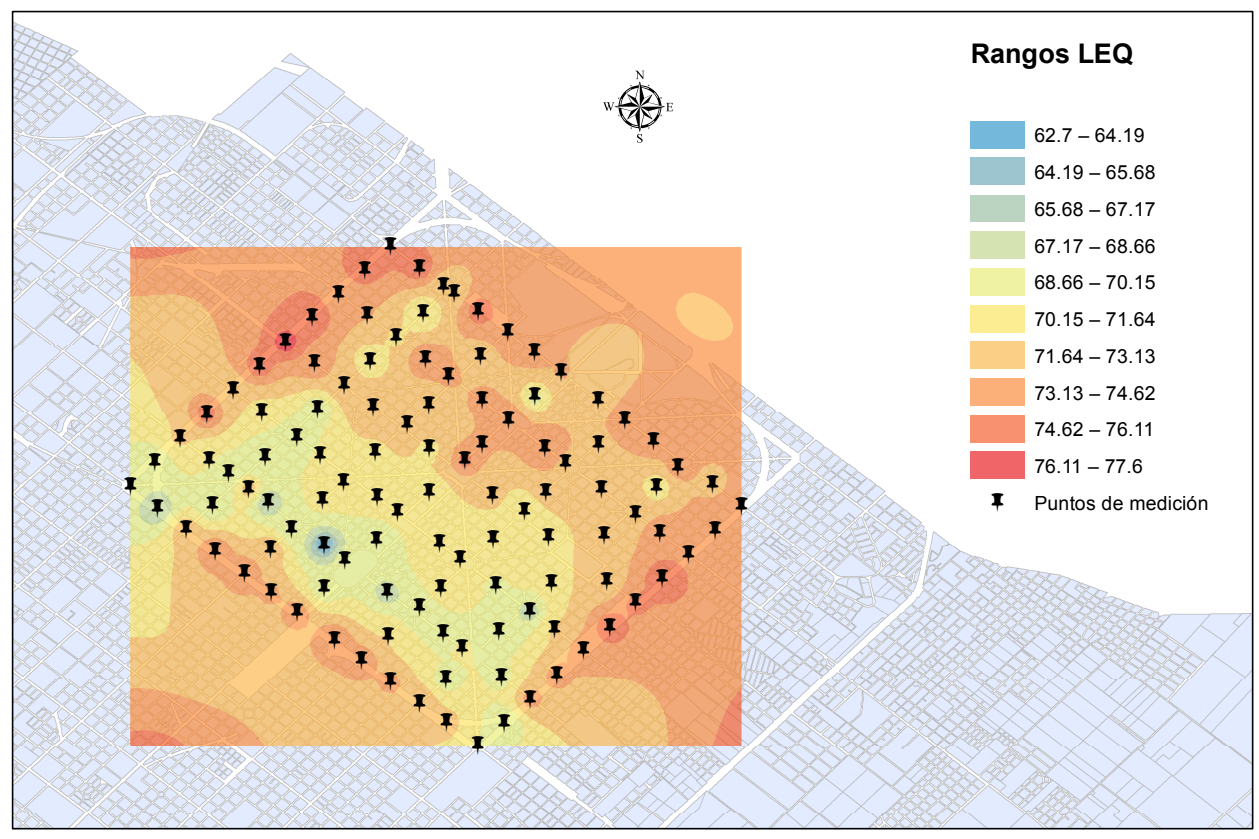




\section{Valuación por cuantiles en la ciudad de Buenos Aires}

\section{Introducción}

En este capítulo, además de las interacciones espaciales, se agrega una complejidad al análisis que tiene que ver con que en el mercado inmobiliario algunas características de las propiedades pueden no tener la misma importancia a través de una determinada distribución de los precios. Específicamente, el valor marginal de una determinada característica puede ser valorada de manera diferente a lo largo de todo el rango de precios. En esta situación, los compradores de casas lujosas pueden asignar un valor diferente que los compradores de casas humildes a cada una de las características de la propiedad.

Se utiliza un enfoque de precios hedónicos para analizar la relación entre los valores inmobiliarios y las características de los inmuebles. Bajo el supuesto de que todos los consumidores son iguales y de que el mercado inmobiliario se encuentra en equilibrio, la derivada de la ecuación de precios hedónicos con respecto a cada variable explicativa es el precio marginal implícito de esa característica por el consumidor representativo. En este contexto, la estimación de de Mínimos Cuadrados Ordinarios (MCO) se ajusta muy bien al caso de consumidores homogéneos, ya que identifica los precios implícitos y determina de manera óptima el precio promedio de una propiedad en un muestra dada. Sin embargo, en el marco de consumidores heterogéneos la utilidad de la regresión por MCO puede ser cuestionada para identificar diferentes segmentos de mercado y sus precios implícitos.

Sungase que hay dos consumidores con diferentes niveles de ingreso: uno de bajo poder adquisitivo y con restricciones de acceso al crédito y otro con alto poder adquisitivo que obviamente no está restringido. El primer comprador no se encuentra en el mercado de casas lujosas, ya que ningún banco le va a financiar el préstamo necesario para comprarla, y el individuo de alto poder adquisitivo no considerará comprar la casa de un hombre de bajos recursos, ya que no proporciona las comodidades deseadas y puede afectar negativamente su deseo de estatus social. Por lo tanto, las personas con altos y bajos recursos puede que ocupen propiedades de diferentes valores, como así también pueden desarrollar gustos específicos por ciertas características del inmueble. Las empresas constructoras y los desarrolladores 
inmobiliarios, conscientes de esta situación, construirán distintos tipos de propiedades para adaptarse a las necesidades percibidas de los distintos consumidores.

Además, algunos atributos relevantes de los inmuebles no son observables, como pueden ser la calidad arquitectónica del barrio, el estatus social del mismo, la calidad de los paisajes y los espacios públicos, etc. y pueden no ser valorados de la misma manera a través de la distribución de precios inmobiliarios. Por ejemplo, si los de mayor poder adquisitivo asignan un valor más alto a las características no observables que los de bajos recursos, entonces las propiedades en los cuantiles superiores de la distribución serán ocupados por las personas de mayores recursos en el equilibrio. Como resultado, los precios implícitos de las características no observables en los cuantiles superiores podrían ser impulsadas por la disposición a pagar del grupo de alto poder adquisitivo. En última instancia, si estas personas de mayores recursos económicos valoran una característica no observable particular, mientras que los de bajos recursos no lo hacen, los precios implícitos de dicha característica en la parte superior e inferior de la distribución de la función de respuesta mostrará signos opuestos y efectos por cuantil.

En un caso extremo, un precio alto de un inmueble deja a los hogares de bajos recursos fuera del mercado previsto de propiedades lujosas y un precio bajo es un elemento disuasorio para la entrada de personas de alto poder adquisitivo. El resultado no es una curva de oferta de características de la vivienda, sino varias curvas que dependen de las características de los compradores. Del mismo modo, habrá varios conjuntos de curvas de demanda de cada característica de la propiedad que resultan en varios conjuntos de precios implícitos de las características del inmueble. Por lo tanto, la tarea principal es identificar las diferentes curvas del mercado y sus precios implícitos. La media condicional de la variable de respuesta proporcionada por la estimación de MCO puede no ser la más representativa en estos casos. En efecto, al igual que la media, no siempre es la medida más representativa de una distribución cuando existen valores extremos o una gran variabilidad en la muestra; la estimación MCO producida por la media condicional, no siempre es la mejor expresión de la relación entre las variables si alguno de los problemas mencionados anteriormente surge. En tales casos, la regresión cuantil ofrece la posibilidad de crear diferentes líneas de regresión para diferentes cuantiles de la variable de respuesta, por medio de un método de estimación que se ve menos afectado por la ocurrencia de dichos problemas. Estas cuestiones, pueden modelizarse mediante una forma funcional distinta de la ecuación de precios hedónicos que considere la posibilidad de la no normalidad de los errores y la heteroscedasticidad en el mercado inmobiliario.

En este capitulo, se aplican técnicas econométricas espaciales con el fin de cuantificar las interrelaciones espaciales y se utiliza la regresión por cuantil para examinar si los precios implícitos de las características de los inmuebles varían a través de la distribución condicional de precios. Se logra una amplia cobertura del mercado inmobiliario, ya que cuentan con datos de casas, de departamentos y de lotes baldíos. 
Los resultados obtenidos muestran una dependencia espacial positiva, que varía por tipo de propiedad, pero que no presenta variaciones importantes entre cuantiles. Además, se observa que algunos atributos se comportan de manera diferente en los distintos niveles de precios lo cual permite concluir que los compradores de propiedades de alto precio parecen dispuestos a pagar un precio distinto por ciertas características de las propiedades que los compradores de propiedades de menor precio.

El documento está organizado de la siguiente manera: en la primera sección se definen algunos aspectos metodológicos de las regresiones por cuantiles espaciales, mientras que en la segunda sección se describen los datos utilizados. La tercera sección define la estrategia empírica, luego se presentan los resultados obtenidos y, finalmente, se exponen las conclusiones.

\subsection{Regresiones espaciales por cuantil}

Durante las últimas décadas, diversas innovaciones metodológicas han sido incorporadas al enfoque de los modelos hedónicos. Una de estas innovaciones ha sido la regresión por cuantil, que data de finales de los años setenta cuando fue desarrollada por Koenker \& Bassett (1978). A pesar de que no es un avance reciente, ya que ha existido durante treinta años, la regresión por cuantil no se ha utilizado tan ampliamente como el método de mínimos cuadrados ordinarios a pesar de que su uso ofrece una amplia gama de ventajas en determinadas circunstancias. La regresión por cuantil representa una alternativa a MCO cuando no se cumplen algunos de los supuestos. Específicamente, el método por cuantil proporciona buenos resultados cuando se enfrentan situaciones de heterocedasticidad y de ausencia de normalidad debido a la presencia de valores extremos.

El mercado inmobiliario puede contener algunos problemas econométricos, tales como heterocedasticidad y autocorrelación espacial que ya fueron analizados en los capítulos anteriores. A dichos problemas se agrega la posibilidad de que la forma funcional de la ecuación de precios hedónicos no sea lineal. Estos problemas pueden ser resueltos por medio de regresiones por cuantiles espaciales, ya que permiten estimar diferentes líneas de regresión para los distintos segmentos de la distribución de precios a través de un método basado en los desvíos absolutos. Esta técnica se ha aplicado recientemente a los modelos de precios hedónicos (McMillen, 2008; McMillen \& Thorsnes, 2006 y Mak et al., 2010) y con especificaciones espaciales en algunos casos (Su \& Yang, 2011; Zietz et al., 2008; Kostov, 2009 y Liao \& Wang, 2012).

A diferencia de la regresión lineal clásica, que estima una función de una media condicional, la regresión por cuantil estima una función cuantil condicional, en el que un cuantil de la distribución condicional de la variable de respuesta se expresa como una función de las covariables. Dado que la regresión por cuantil permite que sus estimaciones varíen con el cuantil correspondiente, es particularmente útil cuando existen estos efectos. Otras de las ventajas de 
la regresión por cuantil es su capacidad superior en el manejo de la heterocedasticidad, valores atípicos, y la heterogeneidad no observada. Koenker \& Hallock (2001) y Koenker (2005) revisan este método con gran nivel de detalle.

El mecanismo para llevar a cabo la regresión por cuantil es similar a la regresión ordinaria. La diferencia radica en que en lugar de buscar el argumento que minimiza (argmin) la suma de los residuos al cuadrado, la regresión por cuantil busca el argumento que minimiza la suma ponderada de los residuos absolutos. La principal ventaja de la utilización de las desviaciones absolutas en lugar de los residuos al cuadrado es el comportamiento del modelo en presencia de valores atípicos en la variable dependiente y, por lo tanto, en el término de error. En tales situaciones, la estimación proporcionada por la regresión por cuantil permanece inalterada por los valores extremos debido a que los errores son penalizados de una manera lineal, mientras que la regresión por MCO está basada en la minimización de los errores al cuadrado, que en realidad concede más importancia a estos valores al ponderarlos de manera cuadrática.

Si se considera una muestra de $\mathrm{N}$ observaciones para la estimación de un modelo de precios hedónicos donde $\mathrm{P}$ es un vector de $N$ por 1 que contiene los precios de las propiedades y X es una matriz de variables explicativas de $N$ por $K$, entonces el problema de minimización en una regresión por cuantil puede ser escrito de la siguiente manera:

$$
\widehat{\beta}_{q}=\arg \min _{\beta_{k} \in \mathbb{R}^{k}} \sum_{n=1}^{N}\left[P_{n-} x_{n} \beta_{q}\right] \omega_{n},
$$

donde $\beta_{q}$ es el vector de coeficientes estimados y el subíndice $q \in(0,1)$ denota el cuantil a estimar. Además, $P_{n}$ es el precio de la enésima observación, $x_{n}$ es la fila enésima de $\mathrm{X}$ y $\omega_{n}$ es el peso de la enésima observación que se define como:

$\omega_{n}=2_{q}$, si $P_{n-} x_{n} \beta_{q}>0, \mathrm{y}$

$\omega_{n}=2-2_{q}$, en caso contrario.

La literatura presenta dos métodos para integrar la regresión por cuantil con los modelos econométricos espaciales. El primero es el modelo de regresión en dos etapas por cuantil (2SQR) propuesto por Kim \& Muller (2004), y el otro es un enfoque de regresión por cuantil de variables instrumentales (IVQR) introducido por Chernozhukovm \& Hansen (2006), adaptado a los modelos espaciales por Kostov (2009). Mientras Kostov (2009) considera implícitamente sólo un tipo interacción espacial, la dependencia espacial, Su \& Yang (2011) amplían los argumentos teóricos de Chernozhukovm \& Hansen (2006) y demuestran la aplicabilidad de su método bajo un diseño general de datos dependientes. La ventaja de 2SQR en la estimación es su simplicidad computacional, en comparación con IVQR. Sin embargo, 2SQR se basa en propiedades asintóticas, mientras IVQR tiene mejores propiedades en muestras finitas (Liao \& Wang, 2012). Si bien estos métodos no son específicos de la econometría espacial, sino que 
están abocados a lidiar con problemas de endogeneidad, amplían la posibilidad de utilizar la regresión por cuantil para estimar un modelo espacial.

Una característica interesante de estos modelos es que estas especificaciones permiten que el parámetro espacial dependa del cuantil y que, por lo tanto, pueda ajustarse a diferentes grados de dependencia espacial en diferentes puntos de la distribución. Por ejemplo, se podría tener dependencia espacial sólo en algunas partes de la distribución de la variable dependiente, pero no en otras. El hecho de tener un modelo con un grado variable de dependencia espacial, depende de los objetivos particulares del análisis y de la naturaleza del problema empírico bajo estudio. Además, dado que el método de regresión cuantil no implica un hipótesis sobre el término de error, este último puede exhibir formas arbitrarias de heterocedasticidad.

\subsection{Datos utilizados}

En este capítulo se trabaja con información inmobiliaria de la ciudad más importante de Argentina $^{1}$. La ciudad de Buenos Aires fue fundada sobre un terreno plano en la orilla del Río de La Plata, durante la época colonial. Se desarrolló como el principal puerto comercial, centro político, financiero y económico. Según el censo de 2010, la ciudad tiene una población de 2890151 habitantes en 1150134 hogares.

La base de datos inmobiliarios de Buenos Aires contiene información de más de seis mil propiedades distribuidas en 47 barrios de la ciudad de Buenos Aires correspondientes a noviembre de 2006. La muestra consta de 3162 departamentos, 2014 casas y 1415 terrenos baldíos. La distribución espacial de los datos utilizados se muestra en las Figuras 4.1, 4.2 y 4.3. Además de las características constructivas usuales, el conjunto de datos contiene información sobre la distancia al centro, la cercanía a avenidas, a escuelas, a zonas verdes, a autopistas y a las estaciones de subte y de ferrocarril. El cuadro 4.1 presenta un resumen de las estadísticas descriptivas desagregadas por tipo de propiedad.

Las casas en la ciudad de Buenos Aires tienen un precio de venta más alto y son de mayor tamaño que los departamentos. Una casa promedio en la ciudad costaba alrededor de 200 mil dólares en 2006 y tenía, en promedio, 225 metros cuadrados, mientras que los departamentos costaban, en promedio, 90 mil dólares y tenían casi 80 metros cuadrados. Un lote promedio costaba casi 450 mil dólares y tenia una superficie de 500 metros cuadrados. El origen relativamente moderno de Buenos Aires explica la gran cantidad de avenidas, en promedio una casa se encuentra a menos de dos cuadras de una avenida y a 9,4 kilómetros del centro. Los departamentos están más cerca de las avenidas, del centro y del transporte público. Los lotes en venta se encuentran, en promedio, a 7.8 kilómetros del centro y a una cuadra de la avenida más cercana. La red de escuelas se encuentra relativamente extendida, por lo tanto no hay mucha variabilidad relativa

\footnotetext{
${ }^{1}$ Los datos de la Ciudad de Buenos Aires fueron utilizados en Cruces et al. (2007) para estimar la calidad de
} vida urbana. 
en este indicador. Por otro lado, la red de subtes tiene una baja cobertura para una ciudad de este tamaño y presenta una mayor variabilidad en la distancia a una estación de subte o de ferrocarril.

Los cuadros 4.7, 4.8 y 4.9 muestran las estadísticas de departamentos, de casas y de terrenos baldíos diferenciando por barrio ${ }^{2}$. Los precios de las casas por metro cuadrado oscilan entre 416 (La Boca) y 2107 (Retiro), en los departamentos van desde 612 (Villa Lugano) a 2810 (Puerto Madero) y en los lotes baldíos los precios oscilan entre 224 (La Boca) y 5914 (Retiro). Dentro de los barrios, hay una variabilidad significativa en los precios, como se puede observar en el cuadro 4.6, que presenta la desviación estándar relativa (RSD) para los precios de cada barrio. La localidad con mayor oscilación en los precios es el barrio de Palermo, una zona de alto valor. Sin embargo, los barrios con mayor desviación respecto a la media son de precio medio (Parque Chacabuco y Floresta) e, inclusive, un barrio de precios bajos (Nueva Pompeya). En el otro extremo, los barrios con mayor igualdad de precio son las urbanizaciones de lujo como Puerto Madero, en el que todas las propiedades tienen altos precios.

\subsection{Estrategia empírica}

La estrategia empírica utilizada comienza con la estimación por MCO de un modelo hedónico básico que relaciona el precio de un inmueble con sus características. Formalmente, se estima el siguiente modelo:

$$
P=X \beta+\mu
$$

donde $P$ es un vector de $N$ por 1 que contiene los precios de las propiedades, $X$ es una matriz de $N$ por $K$ de variables independientes y $\mu$ es el término de error.

Luego, siguiendo los pasos establecidos en el capítulo metodológico, se realizan los test propuestos por Anselin et al. (1996) sobre los errores de la estimación por MCO para detectar la existencia de interacciones espaciales. Dichos test presentados en el cuadro 4.2 muestran que el multiplicador de Lagrange robusto $L M_{\text {lag }}$ es el único significativo al $1 \%$ para todos los tipos de propiedades y, por lo tanto, permite rechazar la hipótesis nula de no autocorrelación espacial en favor de la especificación del modelo de rezago espacial (SLM). Por lo que el modelo que mejor explica el proceso generador de datos tendría la forma SLM especificado de la siguiente manera:

$$
P=\rho_{q} W P+X \beta_{q}+\mu_{q},
$$

${ }^{2}$ El único barrio para los que no hay datos es Parque Chas, que registró ninguna propiedad en el mercado ese mes en particular. 
donde $W P$ es la variable endógena ponderada espacialmente por la matriz $W$, el subíndice $q$ indica el cuantil correspondiente y $\rho_{q}$ y $\beta_{q}$ son los coeficientes a ser estimados. La matriz $W$ captura la vecindad de cada observación según el criterio de distancia definido en la sección 1.3 del capítulo 1.

A diferencia de los capítulos anteriores, para la ciudad de Buenos Aires se cuenta con un muestra de gran tamaño, lo que permite la utilización del método de estimación por cuantiles espaciales en dos etapas (2SQR). En la primera etapa, se estima WP en función de las variables exógenas $X$ y de las variables exógenas ponderadas espacialmente $W X$. Luego, el valor predicho $\hat{W P}$ que se obtiene en la primera etapa de regresión sustituye a $W P$ en el modelo 4.2 para eliminar la correlación entre la variable endógena ponderada espacialmente y el término de error. Finalmente, se realiza la segunda etapa de la regresión por cuantil para obtener $\rho_{q}$ y $\beta_{q}$.

A diferencia del modelo de rezago espacial estándar, el parámetro de dependencia espacial $\rho_{q}$ y el vector de parámetros de regresión $\beta_{q}$ son dependientes del cuantil correspondiente de la variable dependiente. Por lo que, en el análisis empírico, el principal punto de interés serán las derivadas parciales $\left(I-\rho_{q} W\right)^{-1} \beta_{q}$ más que los coeficientes $\beta_{q}$.

Aunque en general la dependencia espacial en el término de error no afecta la consistencia de los estimadores, puede tener un efecto perjudicial en la eficiencia. Sin embargo, este último es un tema que preocupa para la construcción de intervalos de confianza. La ausencia de supuestos sobre la distribución del término de error, que de hecho es la principal ventaja de esta técnica, se convierte en el principal inconveniente cuando se trata de validar el modelo, ya que la matriz de varianza-covarianza de los parámetros que se utiliza para probar su importancia es desconocida. Diferentes métodos para la construcción de intervalos de confianza pueden ser empleados, algunos de cuales requieren supuestos más fuertes (como por ejemplo la distribución i.i.d. de los errores), mientras que otros no lo requieren como el método Bootstrap. En este capitulo, la significatividad estadística se obtiene mediante un procedimiento de bootstrap con 500 repeticiones.

\subsection{Resultados de la estimación}

En esta sección se analiza la relación entre el precio de venta de los inmuebles y los atributos que conforman el paquete de características (tamaño del lote, antigüedad, número de habitaciones y de baños, garaje, etc.), a través de la estimación del modelo de precios hedónicos espaciales por cuantil. En las regresiones realizadas, se utiliza el logaritmo del precio como variable dependiente. Además de los atributos del inmueble, se incluye un conjunto de variables dummy por barrio e indicadores de las características socioeconómicas de la zona. Las siguientes subsecciones presentan los resultados de la estimación de departamentos, de casas y de terrenos baldíos. 


\subsubsection{Departamentos}

En este apartado, se presentan los resultados de la estimación de departamentos de Buenos Aires. En la primera columna del cuadro 4.3 se presenta el modelo espacial, y las columnas restantes presentan los resultados del modelo espacial por cuantil.

La Figura 4.4 muestra cómo los efectos marginales estimados de las variables explicativas varían en la distribución condicional de precios. Las figuras 4.5 y 4.6 hacen lo propio para las distancias a las amenidades y las características socioeconómicas de la zona, respectivamente. Cada uno de estos paneles grafica las estimaciones de los efectos marginales del modelo Spatial Lag y del modelo 2SQR de las variables explicativas y sus intervalos de confianza del $95 \%$.

La estimación del coeficiente que mide la dependencia espacial es de 0,169, lo que indica autocorrelación espacial significativa y positiva. El coeficiente de dependencia espacial presenta una tendencia creciente que se incrementa en los últimos cuantiles. Sin embargo, los intervalos de confianza del modelo Spatial lag y 2SQR se superponen, por lo que no se puede afirmar que los coeficientes de ambos modelos sean distintos.

Se puede observar una tendencia creciente y significativa de la superficie total que cruza el intervalo de confianza del modelo Spatial Lag. El número de habitaciones es significativo sólo para los departamentos de mayor precio. Como resultado, un dormitorio adicional tendrá un valor marginal mayor en los departamentos de mayor precio. El signo negativo para la antigüedad y el signo positivo para la antigüedad al cuadrado sugieren que los departamentos más nuevos tienen precios más altos. Hay una prima más alta para los departamentos nuevos en los hogares de mayor precio. En lo que respecta a los baños, los valores de las viviendas de mayor y de menor precio están influidos positivamente por el número de cuartos de baño; el coeficiente tiene un patrón en forma de U.

En el cuadro 4.3 se observa que la distancia a avenidas, a espacios verdes y a escuelas no es significativa. Los resultados muestran que la distancia a la autopista presenta un efecto más negativo en los cuantiles más altos, lo que indica que los departamentos de precios más altos valoran más estar cerca de los accesos al centro de la ciudad. La distancia al centro comercial muestra una tendencia mayor en los cuantiles más bajos, lo que indica que los departamentos de precios más bajos valoran más estar cerca del centro. La distancia a la estación de tren no es significativa en la mayoría de los cuantiles.

$\mathrm{Al}$ analizar las características socioeconómicas de los barrios, se encuentra que sólo la tasa de empleo es relevante, aunque no es significativa en todos los cuantiles. Adicionalmente, la cobertura de salud es relevante sólo para los departamentos más baratos. El salario horario medio, la proporción de propietarios, el acceso a la recolección de residuos y al transporte público y la proporción de trabajadores informales en la zona no parecen ser determinantes del precio de los departamentos de Buenos Aires. 


\subsubsection{Casas}

El análisis de los resultados de la estimación de casas se presenta en el cuadro 4.4, mientras que las figuras 4.7, 4.8 y 4.9 muestran la variación de los coeficientes de los distintos atributos, de las distancias a amenidades y de las características socioeconómicas de la zona a través de la distribución condicional de precios, respectivamente.

El coeficiente que mide la dependencia espacial presenta un valor significativo de 0,11 en el modelo Spatial lag, el cual no presenta variaciones importantes entre cuantiles. El valor positivo indica una autocorrelación espacial positiva significativa en los precios de las casas de Buenos Aires. Una casa en un barrio determinado tiende a verse influenciada levemente por los precios de las casas vecinas.

La relación entre la superficie cubierta y el precio de las viviendas presenta una forma de U invertida entre cuantiles y una clara tendencia creciente de la superficie del lote (ambas relaciones cruzan los intervalos de confianza del modelo Spatial Lag). El coeficiente que acompaña la superficie del lote indica que las casas más caras valoran más un metro cuadrado adicional de terreno. El número de dormitorios no presenta una relación significativa. Por otro lado, el signo negativo de la antigüedad y el signo positivo para la antigüedad al cuadrado sugieren que las casas más nuevas se venden relativamente a mayores precios y esa relación no varía por cuantiles. Con respecto al coeficiente relacionado con los cuartos de baño, los resultados obtenidos sugieren que las casas de precios más bajos asignan un mayor valor a los cuartos de baño adicionales. Algo similar sucede con el lugar para estacionar: las casas de los cuantiles más bajos asignan un mayor valor al garaje que las casas de los cuantiles más altos, en los cuales la variable garaje ni siquiera es significativa. El efecto marginal del número de plantas también varía por cuantil, lo cual siguiere que las casas de precios más altos asignan un mayor valor a una planta adicional. Las variables de distancia no resultan ser significativas, solo la distancia a las avenidas es significativa para los cuantiles más bajos, lo que indica que en las casas de menor valor se castiga de mayor medida el ruido y la contaminación.

Al analizar las características socioeconómicas de los barrios, se encuentra que el salario promedio de la zona es significativo si se analiza la totalidad de las casas, pero al analizar por cuantil se observa que solo es significativa y positiva para los cuantiles más altos. Un comportamiento similar se presenta con la tasa de desempleo de la zona, que si bien no es significativa para la totalidad de las casas, sí lo es para los cuantiles más bajos. El trabajo informal es valorado negativa y significativamente por los cuantil más altos, mientras que la cobertura de salud es valorada positiva y significativamente por los cuantiles más bajos. La proporción de propietarios, el acceso a la recolección de residuos y al transporte público no parecen ser determinantes del precio de las casas en Buenos Aires. 


\subsubsection{Lotes baldíos}

En este apartado, se presentan los resultados de la estimación de los lotes baldíos. El cuadro 4.5 presenta los resultados de las estimaciones y las figuras 4.10, 4.11 y 4.12 muestran cómo varían los coeficientes de los atributos, de las distancias a amenidades y de las características socioeconómicas de la zona a través de la distribución condicional de precios, respectivamente. La dependencia espacial tiene una valor significativo de 0,29 si se analiza la totalidad de los lotes en venta y presenta una tendencia negativa entre cuantiles. Como los intervalos de confianza se superponen, no se puede concluir que los coeficientes de ambos modelos sean diferentes. La superficie del lote muestra una tendencia significativa y positiva en los cuantiles.

Al analizar la información sobre los códigos urbanísticos, más precisamente el factor de ocupación total permitido por el gobierno, se observa que dicha característica es significativa al $5 \%$ si se incluyen todos los lotes. Sin embargo, al analizar por cuantil se encuentra una alta significatividad y un efecto positivo sobre los cuantiles más bajos.

Los resultados de las estimaciones de distancia indican que la cercanía a una avenida tiene una fuerte tendencia alcista (con coeficientes negativos), lo que indica que una mayor distancia es penalizada de mayor manera por los lotes de los cuantiles más altos. Algo similar ocurre con la distancia al subte; se indica la relevancia de los accesos a vías de comunicación en los lotes, sobre todo en los lotes más caros. La distancia a escuelas muestra una ligera tendencia al alza (también coeficientes negativos), lo que indica que los lotes de precios más altos penalizan más la lejanía a una institución educativa. La distancia al centro, a áreas verdes, a la autopista y a las estaciones de ferrocarril no parecen ser determinantes del precio de los lotes en Buenos Aires.

$\mathrm{Al}$ analizar las características socioeconómicas de los barrios se encuentra que, si bien el salario promedio de la zona es significativo al analizar la totalidad de los lotes, teniendo en cuenta los cuantiles sólo es significativo y positivo para los cuantiles más altos. Adicionalmente, la cobertura de salud y el trabajo informal resultan significativos para los cuantiles medios. El resto de las características de la zona no son significativas.

\subsection{Conclusiones}

En esta capítulo se estimó un modelo espacial por cuantil para analizar el hecho de que algunas características de los inmuebles pueden ser valoradas de manera distinta a lo largo de distribución de los precios, teniendo en cuenta la existencia de las interacciones espaciales. La base de datos utilizada contiene departamentos, casas y terrenos baldíos, lo que permite abarcar buena parte del mercado inmobiliario.

Los resultados muestran que el efecto de las distintas características de una propiedad sobre el precio de venta puede ser mejor explicado por la estimación de regresiones por cuantil espaciales. 
El trabajo muestra la existencia de diferentes comportamientos de la dependencia espacial en los distintos tipos de propiedades y que dicha dependencia no varía entre cuantiles.

Además, los coeficientes de regresión de algunas variables se comportan de manera diferente en los distintos niveles de precios, lo cual permite concluir que los compradores de propiedades de altos precio parecen dispuestos a pagar un precio distinto por ciertas características de las propiedades que los compradores de propiedades de precios más bajos. 


\subsection{Apéndice}

Cuadro 4.1: Estadísticas descriptivas

\begin{tabular}{|c|c|c|c|c|c|c|}
\hline \multirow{2}{*}{ Variables } & \multicolumn{2}{|r|}{ Casas } & \multicolumn{2}{|c|}{ Departamentos } & \multicolumn{2}{|r|}{ Lotes } \\
\hline & Media & Desvío estándar & Media & Desvío estándar & Media & Desvío estándar \\
\hline Precio (USAD) & 205,067 & {$[4490]$} & 92,638 & {$[2069]$} & 444,306 & {$[28903]$} \\
\hline Superficie cubierta (mts) & 225.4 & [2.913] & 78.2 & {$[0.902]$} & & \\
\hline Superficie del lote (mts) & 241.5 & {$[3.088]$} & & & 510.8 & {$[23.466]$} \\
\hline Dormitorios & 3.4 & {$[0.03]$} & 1.7 & {$[0.021]$} & & \\
\hline Factor de ocupación total & & & & & 0.54 & {$[1.30]$} \\
\hline Antigüedad & 32.7 & {$[0.46]$} & 13.2 & {$[0.384]$} & & \\
\hline Baños & 2.5 & {$[0.029]$} & 0.6 & {$[0.018]$} & & \\
\hline Número de plantas & 1.9 & {$[0.016]$} & & & & \\
\hline Garaje & 0.8 & {$[0.01]$} & & & & \\
\hline Distancia a la avenida & 0.2 & {$[0.003]$} & 0.1 & {$[0.002]$} & 0.1 & {$[0.003]$} \\
\hline Distancia a la escuela & 0.2 & {$[0.002]$} & 0.1 & {$[0.002]$} & 0.2 & {$[0.003]$} \\
\hline Distancia a espacio verde & 0.3 & {$[0.004]$} & 0.3 & {$[0.003]$} & 0.3 & {$[0.005]$} \\
\hline Distancia a la autopista & 1.4 & {$[0.025]$} & 1.8 & {$[0.019]$} & 1.7 & {$[0.029]$} \\
\hline Distancia al centro & 9.4 & {$[0.067]$} & 6.0 & {$[0.071]$} & 7.8 & {$[0.089]$} \\
\hline Distancia al subte & 2.5 & {$[0.038]$} & 1.2 & {$[0.025]$} & 1.8 & {$[0.04]$} \\
\hline Distancia a la estación de tren & 1.1 & {$[0.012]$} & 1.0 & {$[0.01]$} & 1.0 & {$[0.013]$} \\
\hline Salario medio horario & 516 & {$[0.655]$} & 571 & {$[0.588]$} & 518 & {$[0.76]$} \\
\hline Tasa de desempleo & $20.6 \%$ & {$[12]$} & $11.8 \%$ & [8] & $19.5 \%$ & {$[13]$} \\
\hline Proporción de propietarios & $72.7 \%$ & {$[23]$} & $66.6 \%$ & {$[21]$} & $68.5 \%$ & {$[30]$} \\
\hline Tasa acceso a recolección de residuos & $99.4 \%$ & {$[10]$} & $99.3 \%$ & {$[12]$} & $99.6 \%$ & {$[6]$} \\
\hline Tasa acceso a transporte público & $99.4 \%$ & {$[10]$} & $99.3 \%$ & {$[13]$} & $99.6 \%$ & [6] \\
\hline Tasa de trabajo informal & $98.2 \%$ & {$[3]$} & $98.4 \%$ & {$[2]$} & $98.3 \%$ & {$[4]$} \\
\hline Tasa de personas sin cobertura de salud & $58.9 \%$ & {$[27]$} & $77.3 \%$ & {$[25]$} & $59.1 \%$ & {$[31]$} \\
\hline
\end{tabular}

Distancias en kilómetros

Figura 4.1: Distribución de las propiedades: Departamentos

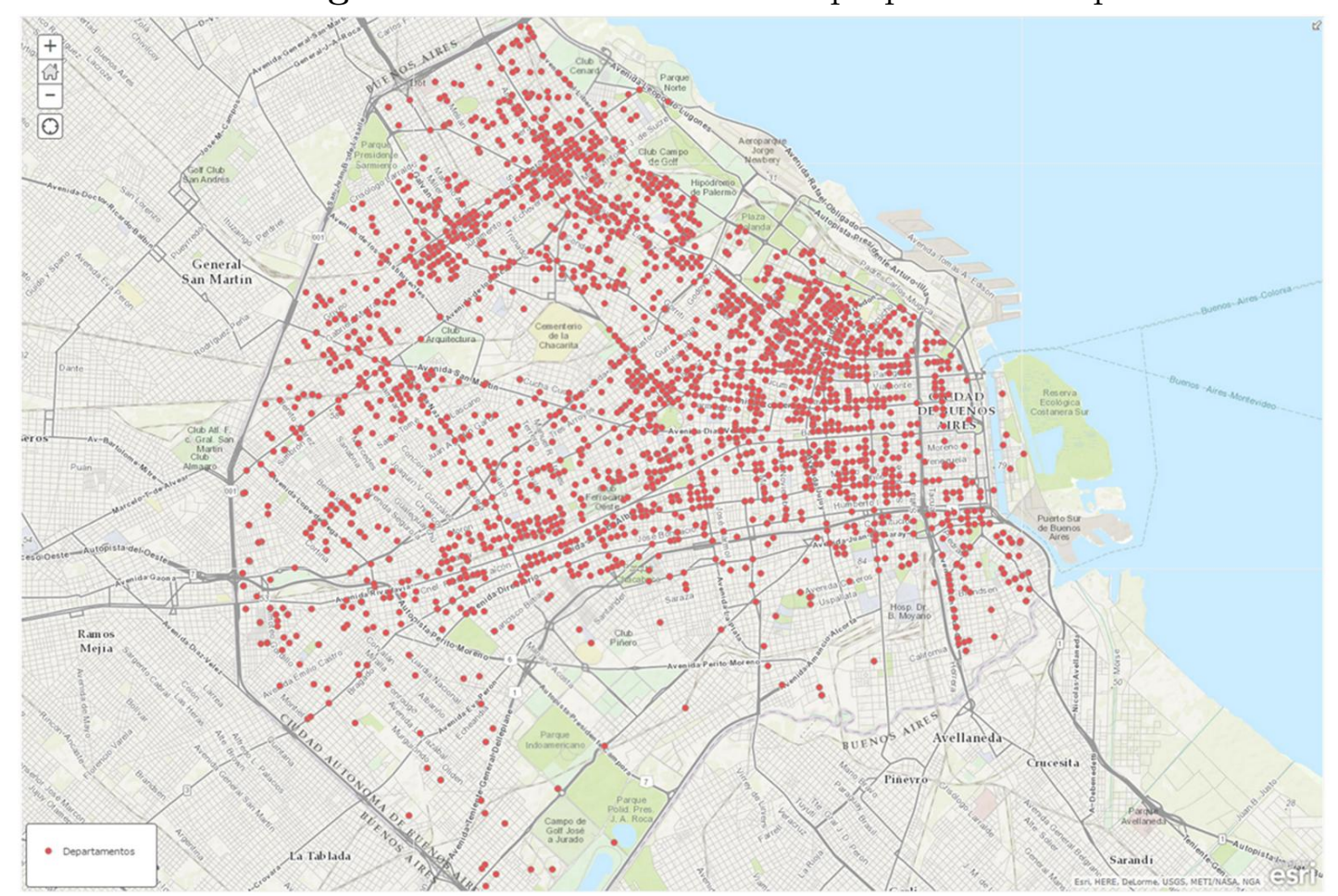


Figura 4.2: Distribución de las propiedades: Casas

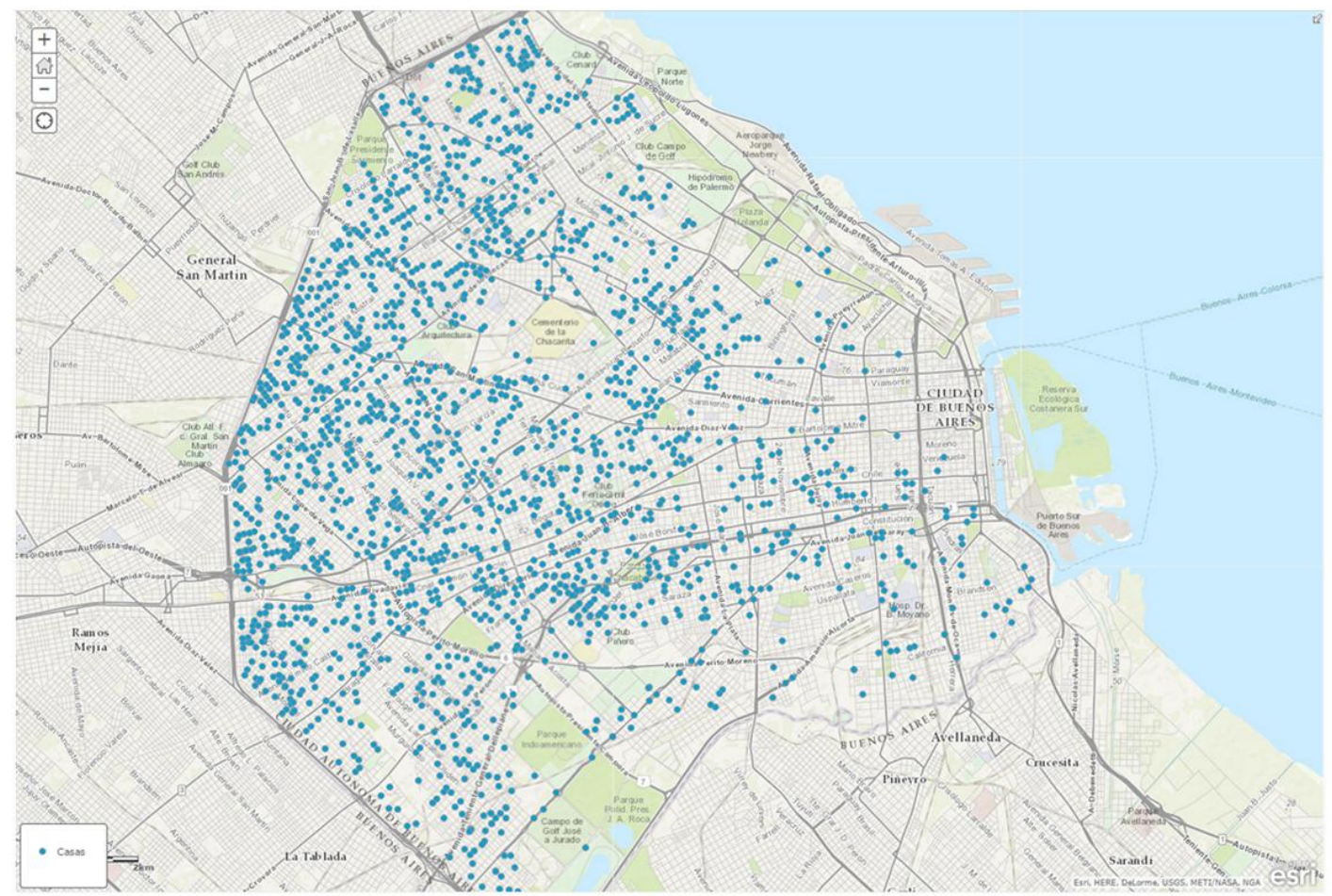

Figura 4.3: Distribución de las propiedades: Lotes

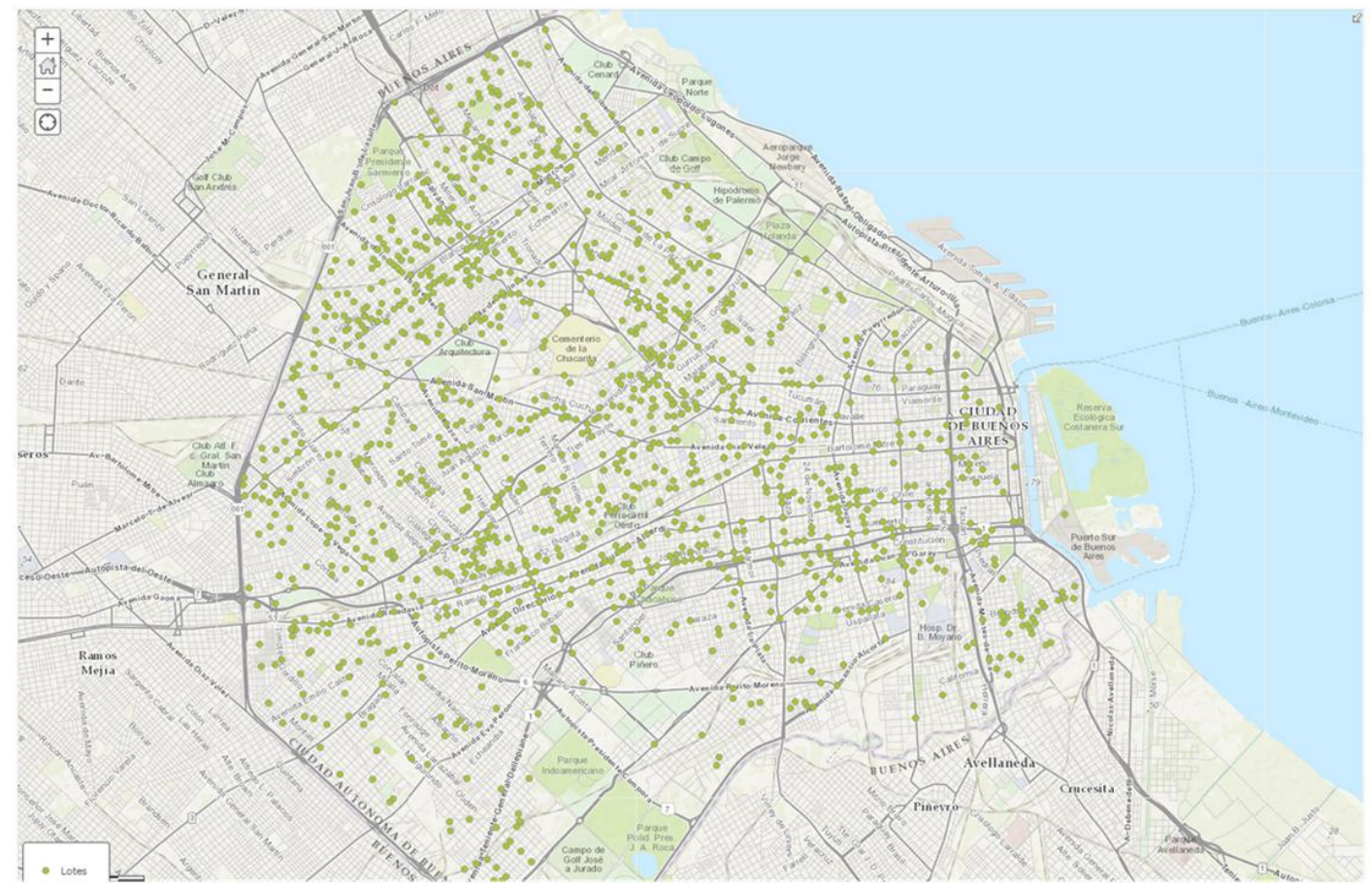


Cuadro 4.2: Diagnostico Espacial

\begin{tabular}{cccc}
\hline \multirow{2}{*}{ Tipo de propiedad } & \multicolumn{3}{c}{ Estadísticos } \\
\cline { 2 - 4 } & I de Moran & Multiplicador de Lagrange & Multiplicador de Lagrange Robusto \\
\hline \multirow{2}{*}{ Departamentos } & $2.818^{* * *}$ & 2.588 & 1.75 \\
& & $14.553^{* * *}$ & $13.715^{* * *}$ \\
\hline \multirow{2}{*}{ Casas } & $7.598^{* * *}$ & $43.988^{* * *}$ & 1.751 \\
& & $90.602^{* * *}$ & $48.366^{* * *}$ \\
\hline \multirow{2}{*}{ Lotes } & $5.464^{* * *}$ & $16.69^{* * *}$ & $3.242^{*}$ \\
& & $56.324^{* * *}$ & $42.876^{* * *}$ \\
\hline
\end{tabular}

$*$ significativo al 10\%; ** significativo al 5\%; *** significativo al 1\% 


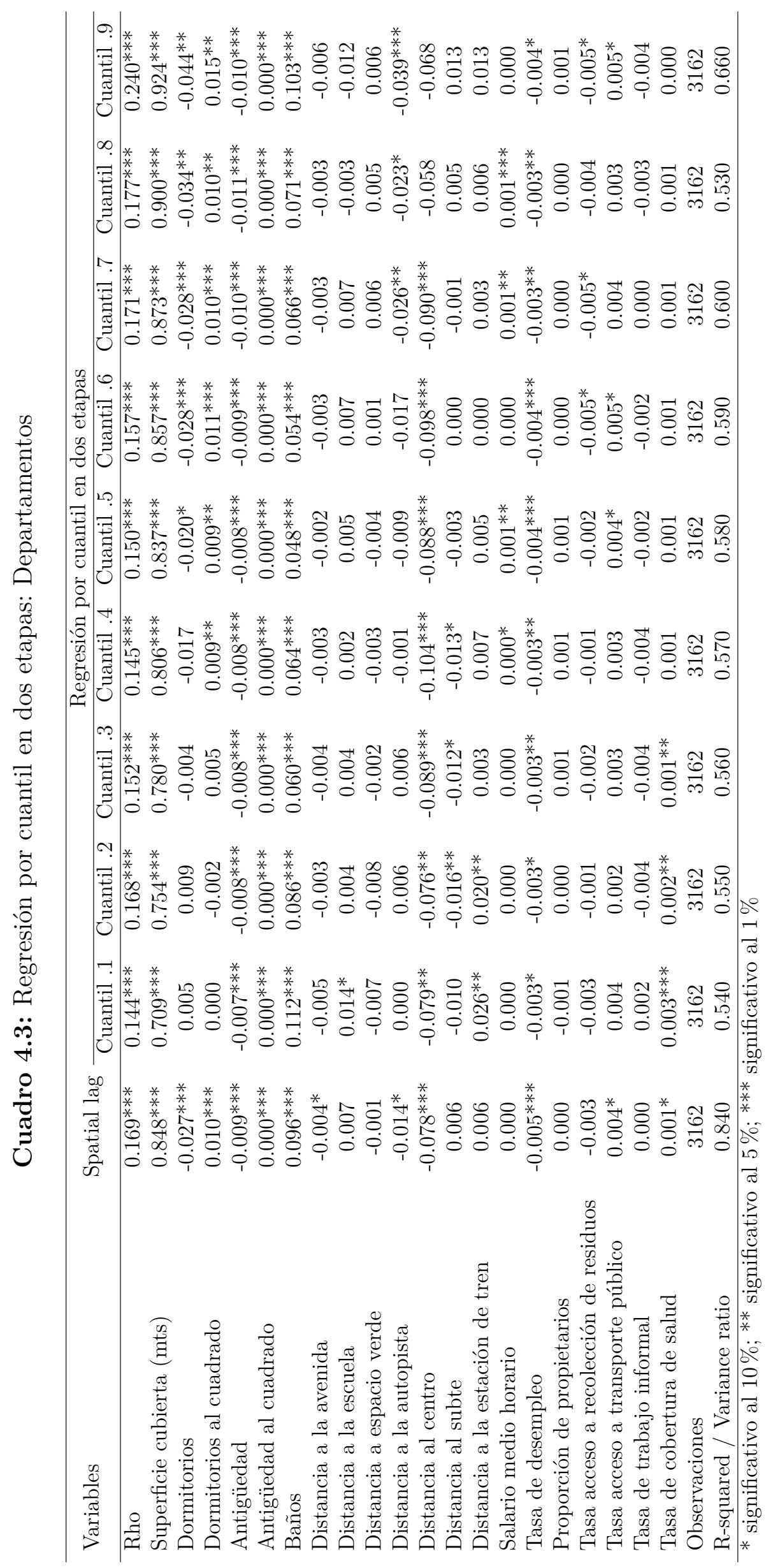




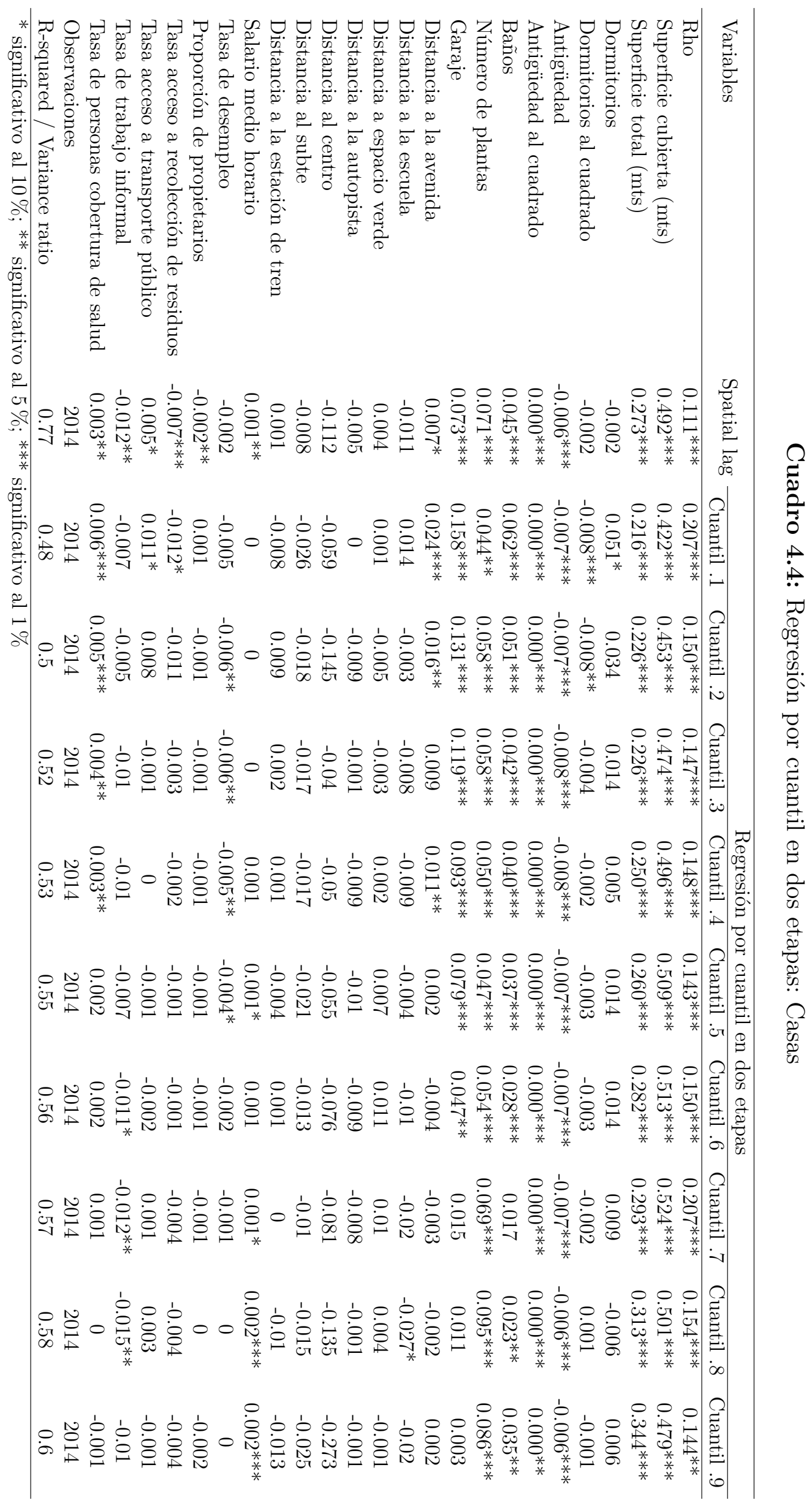




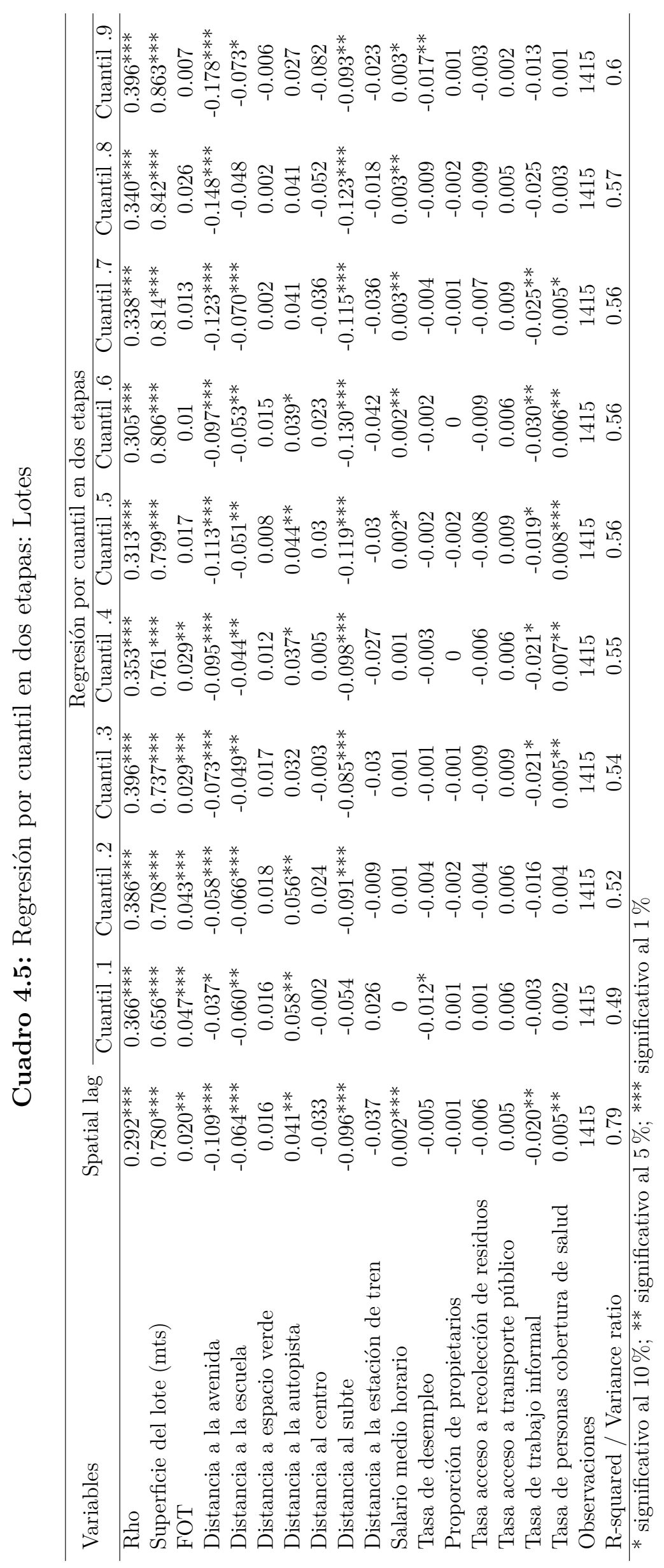


Cuadro 4.6: Desviación estándar relativa del precio de los inmuebles

\begin{tabular}{|c|c|c|c|}
\hline Barrio & Casas & Departamentos & Lotes \\
\hline Agronomía & 35.5 & 124.3 & 15.5 \\
\hline Almagro & 32.8 & 145.0 & 16.7 \\
\hline Balvanera & 31.9 & 85.7 & 23.5 \\
\hline Barracas & 37.1 & 90.7 & 22.9 \\
\hline Belgrano & 30.2 & 238.1 & 22.2 \\
\hline Boca & 50.3 & 148.4 & 25.9 \\
\hline Boedo & 28.0 & 109.6 & 18.2 \\
\hline Caballito & 32.0 & 78.2 & 18.3 \\
\hline Chacarita & 35.0 & 58.3 & 15.1 \\
\hline Coghlan & 17.4 & 73.7 & 14.5 \\
\hline Colegiales & 24.0 & 164.2 & 16.7 \\
\hline Constitución & 17.0 & 54.9 & 20.6 \\
\hline Flores & 31.6 & 156.3 & 17.0 \\
\hline Floresta & 45.2 & 151.4 & 42.0 \\
\hline Liniers & 30.1 & 102.6 & 25.8 \\
\hline Mataderos & 34.9 & 59.2 & 19.7 \\
\hline Monte Castro & 23.5 & 95.7 & 15.4 \\
\hline Monserrat & 85.9 & 101.7 & 20.2 \\
\hline Nueva Pompeya & 57.7 & 108.1 & 32.1 \\
\hline Núñez & 27.6 & 112.9 & 21.9 \\
\hline Palermo & 55.7 & 108.6 & 36.0 \\
\hline Parque Avellaneda & 32.6 & 47.3 & 23.3 \\
\hline Parque Chacabuco & 53.8 & 106.5 & 13.1 \\
\hline Parque Patricios & 34.8 & 59.5 & 19.7 \\
\hline Paternal & 23.9 & 69.2 & 21.3 \\
\hline Puerto Madero & 0.0 & 92.5 & 20.1 \\
\hline Recoleta & 47.4 & 103.8 & 29.9 \\
\hline Retiro & 0.0 & 86.3 & 36.2 \\
\hline Saavedra & 26.2 & 71.9 & 23.6 \\
\hline San Cristóbal & 29.0 & 118.7 & 19.0 \\
\hline San Nicolás & 0.0 & 136.8 & 27.3 \\
\hline San Telmo & 19.6 & 81.8 & 16.8 \\
\hline Vélez Sarsfield & 29.9 & 111.4 & 14.9 \\
\hline Versalles & 33.1 & 0.0 & 30.2 \\
\hline Villa Crespo & 38.8 & 126.0 & 18.3 \\
\hline Villa del Parque & 23.3 & 115.3 & 17.0 \\
\hline Villa Devoto & 33.3 & 182.6 & 20.2 \\
\hline Villa Gral. Mitre & 41.4 & 84.7 & 17.2 \\
\hline Villa Lugano & 37.0 & 141.0 & 30.8 \\
\hline Villa Luro & 28.4 & 119.7 & 21.7 \\
\hline Villa Ortuzar & 33.8 & 112.9 & 19.6 \\
\hline Villa Pueyrredón & 31.0 & 38.6 & 15.9 \\
\hline Villa Real & 35.1 & 89.4 & 19.5 \\
\hline Villa Riachuelo & 46.6 & 114.4 & 23.1 \\
\hline Villa Santa Rita & 32.4 & 68.3 & 16.0 \\
\hline Villa Soldati & 35.4 & 22.2 & 35.8 \\
\hline Villa Urquiza & 32.0 & 103.2 & 17.4 \\
\hline Total & 33.8 & 116.3 & 22.8 \\
\hline
\end{tabular}


Figura 4.4: Departamentos, efectos por cuantil
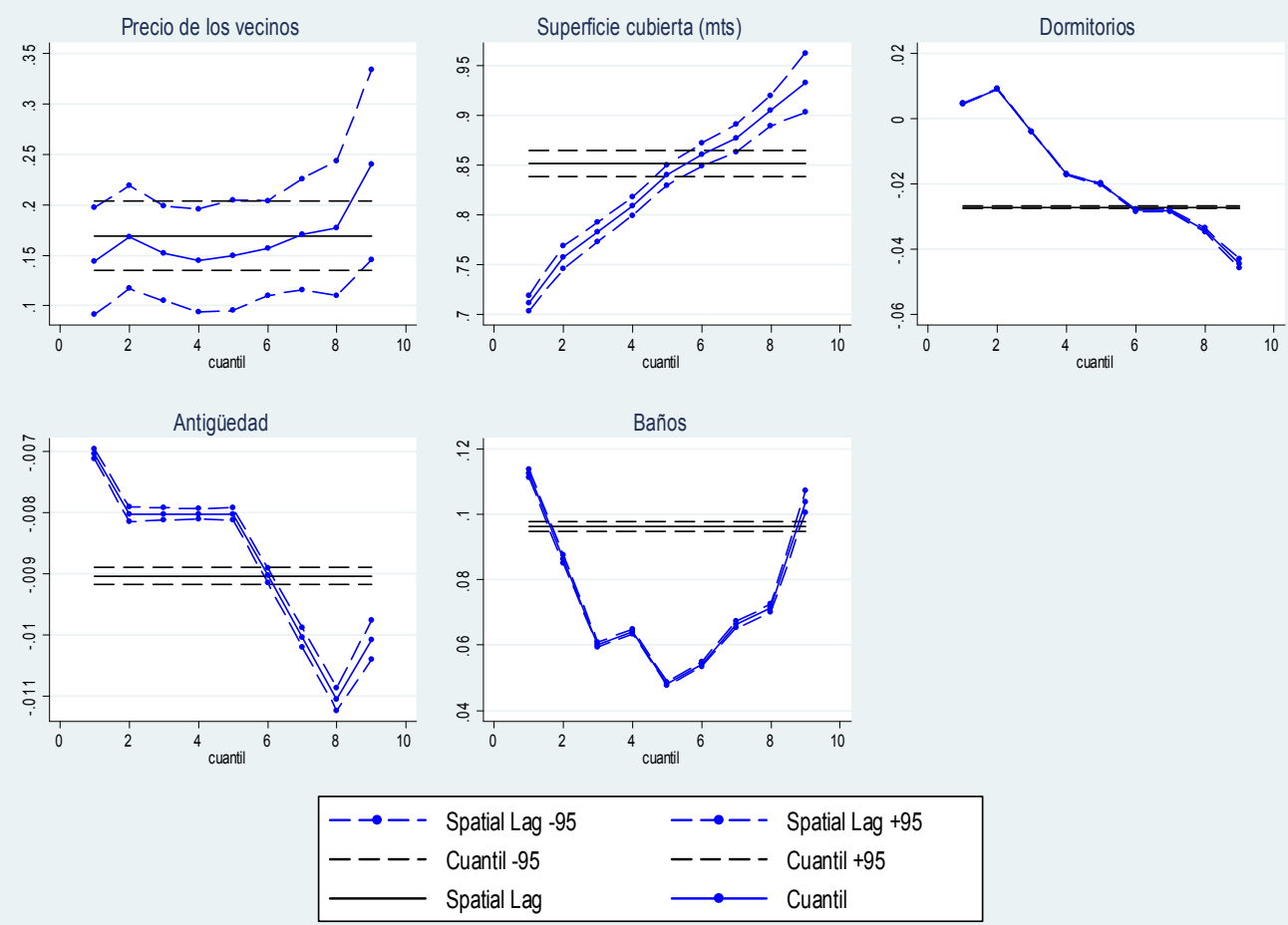

Figura 4.5: Departamentos, efectos por cuantil: Distancias
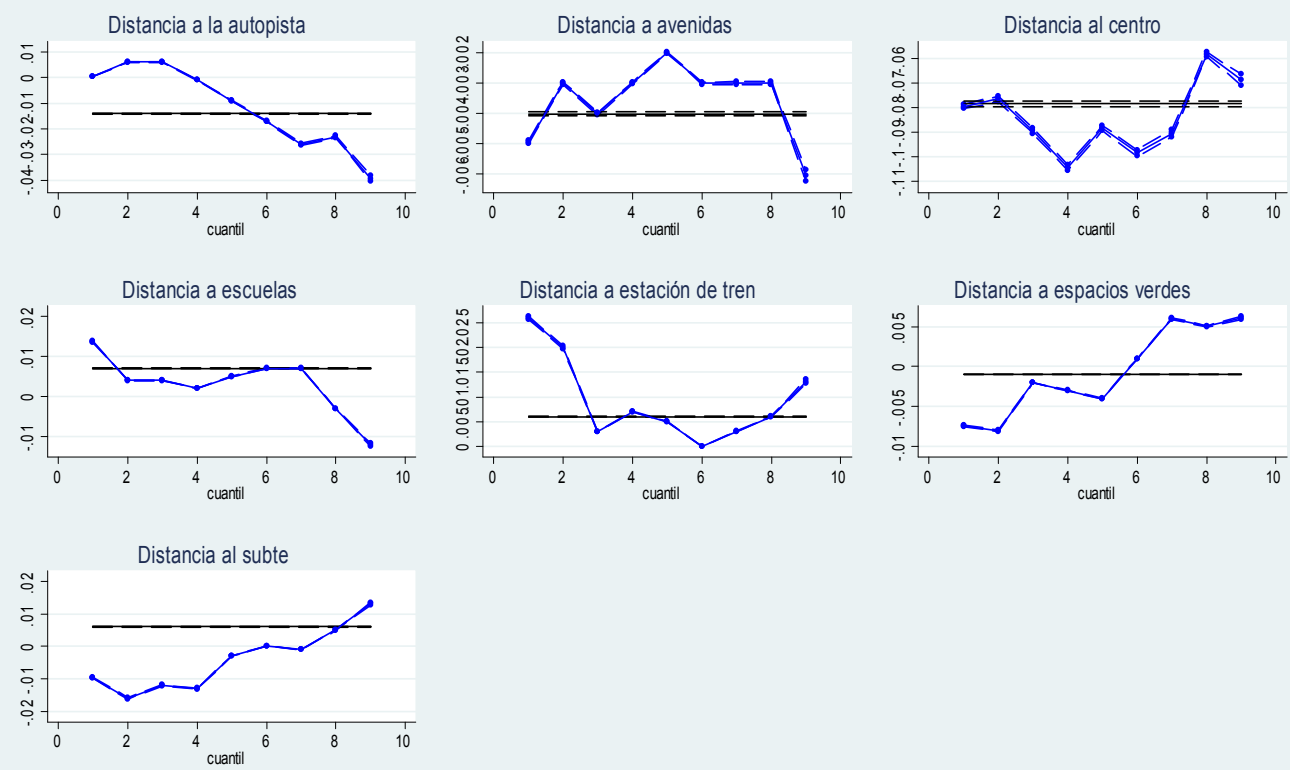

\begin{tabular}{|c|c|c|}
\hline \multirow{2}{*}{\multicolumn{3}{|c|}{ —ーー - Spatial Lag -95 }} \\
\hline & & \\
\hline--- & Cuantil -95 & ---- Cuantil +95 \\
\hline & Spatial Lag & $\longrightarrow$ Cuantil \\
\hline
\end{tabular}


Figura 4.6: Departamentos, efectos por cuantil: Variables socioeconómicas
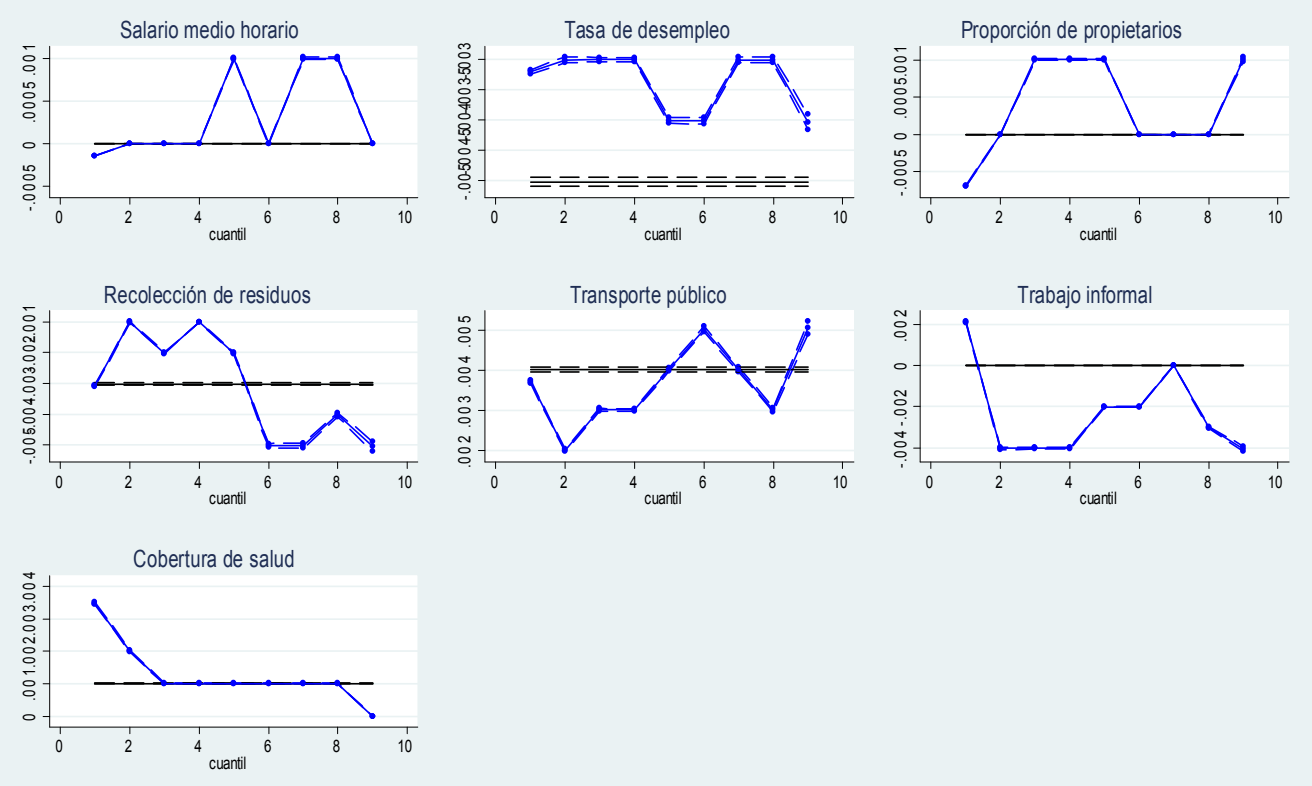

\begin{tabular}{|c|c|c|}
\hline $\begin{array}{l}-\rightarrow-- \\
---- \\
\end{array}$ & $\begin{array}{l}\text { Spatial Lag }-95 \\
\text { Cuantil -95 } \\
\text { Spatial Lag }\end{array}$ & $\begin{array}{ll}-\rightarrow-- & \text { Spatial Lag }+95 \\
---- & \text { Cuantil }+95 \\
\longrightarrow & \text { Cuantil }\end{array}$ \\
\hline
\end{tabular}

Figura 4.7: Casas: efectos por cuantil
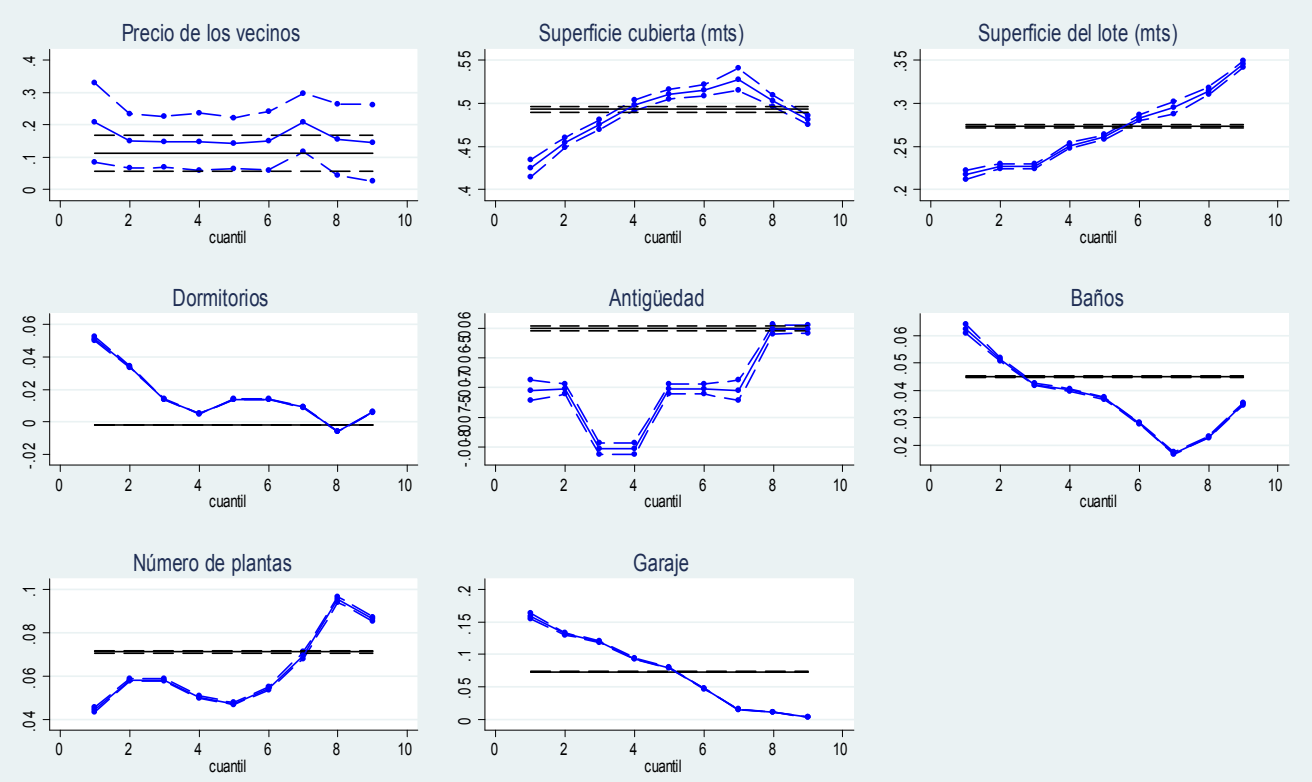

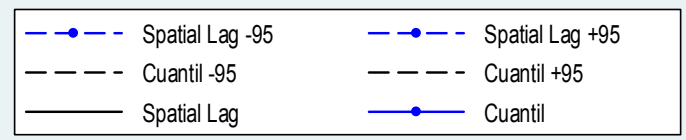


Figura 4.8: Casas, efectos por cuantil: Distancias
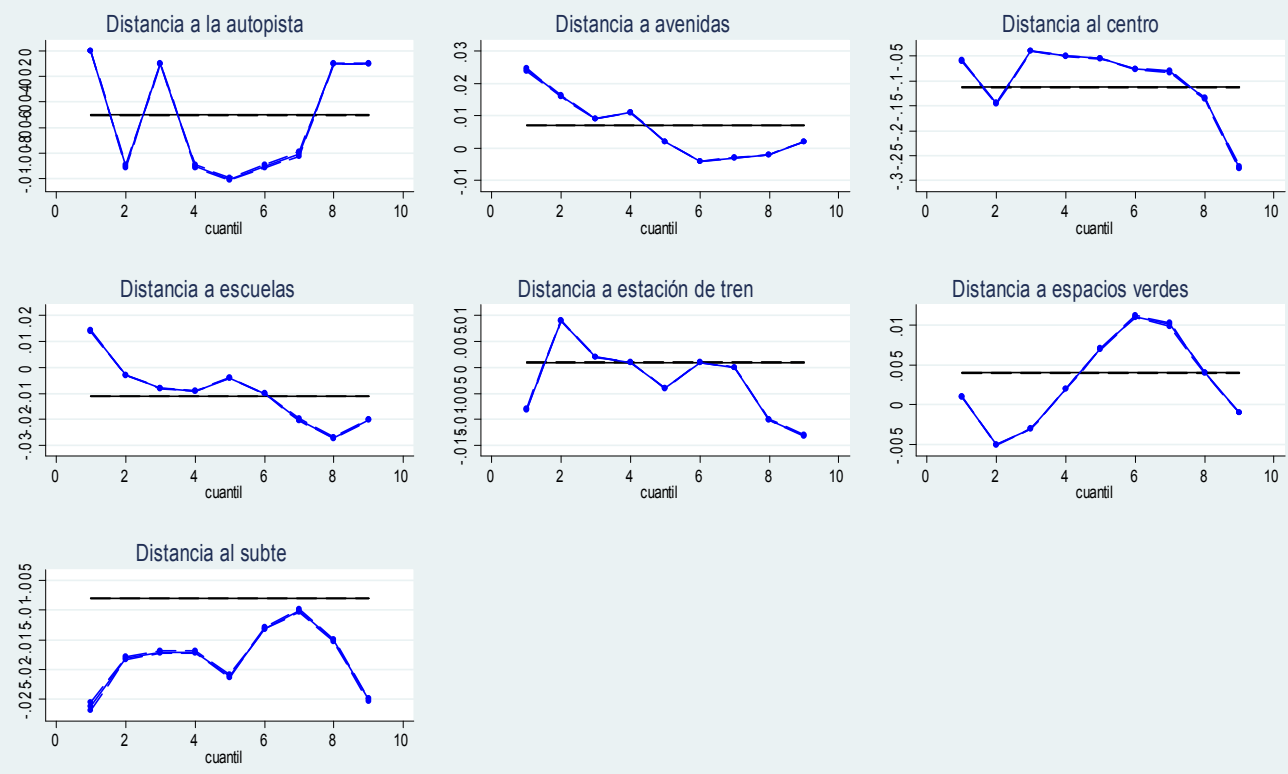

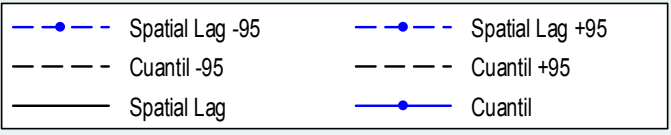

Figura 4.9: Casas, efectos por cuantil: Variables socioeconómicas
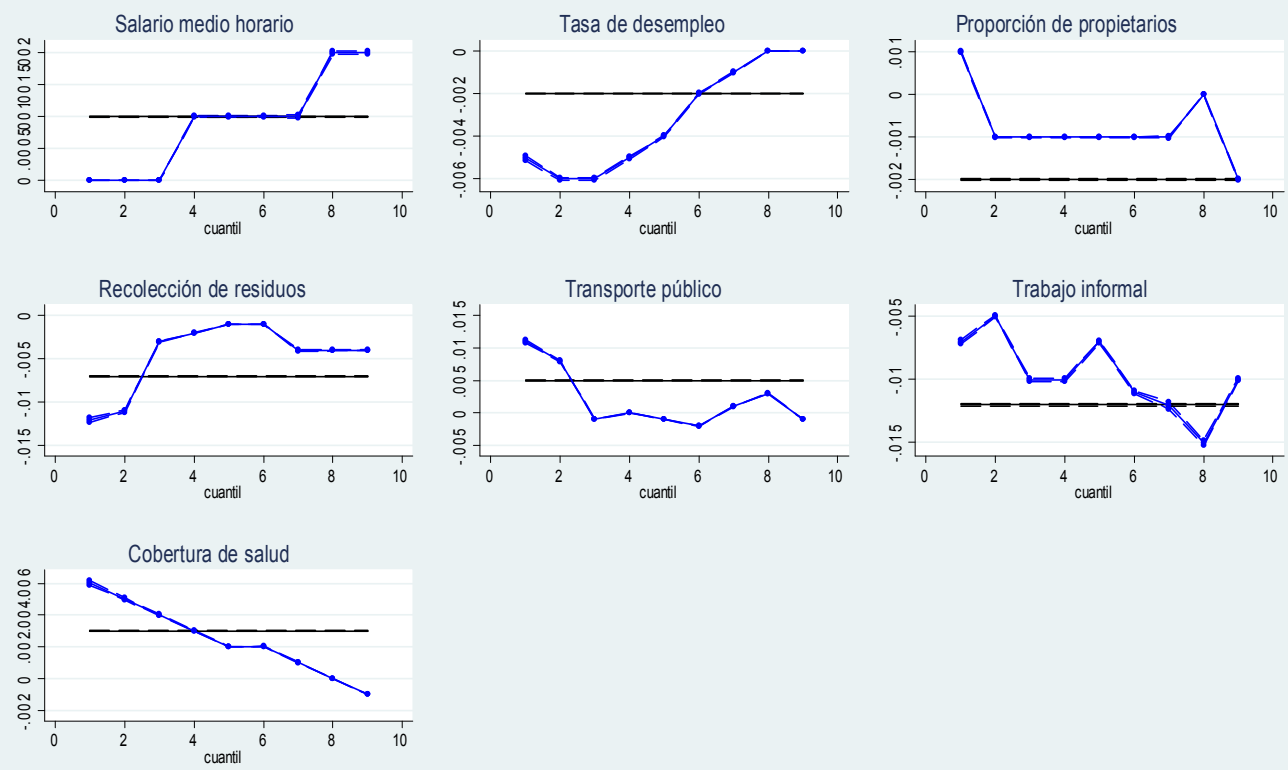

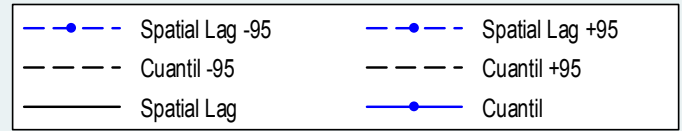


Figura 4.10: Lotes, efectos por cuantil
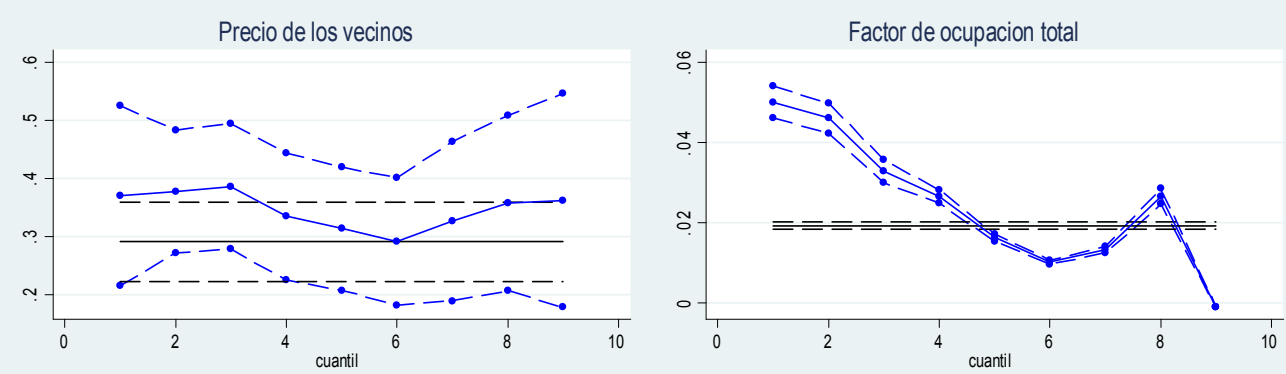

Superficie del lote (mts)

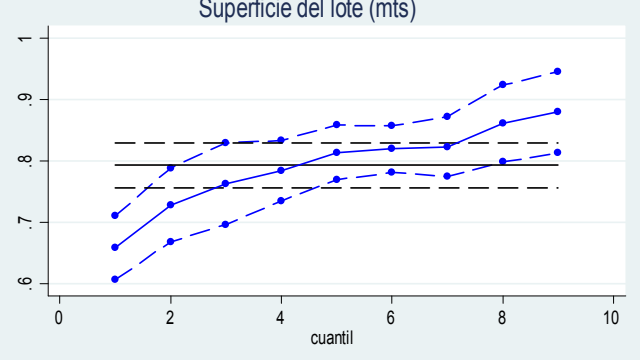

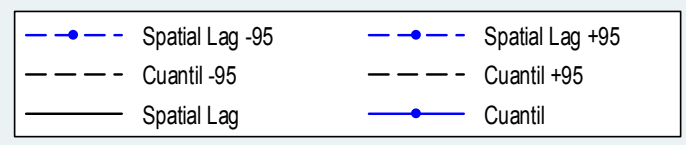

Figura 4.11: Lotes, efectos por cuantil: Distancias
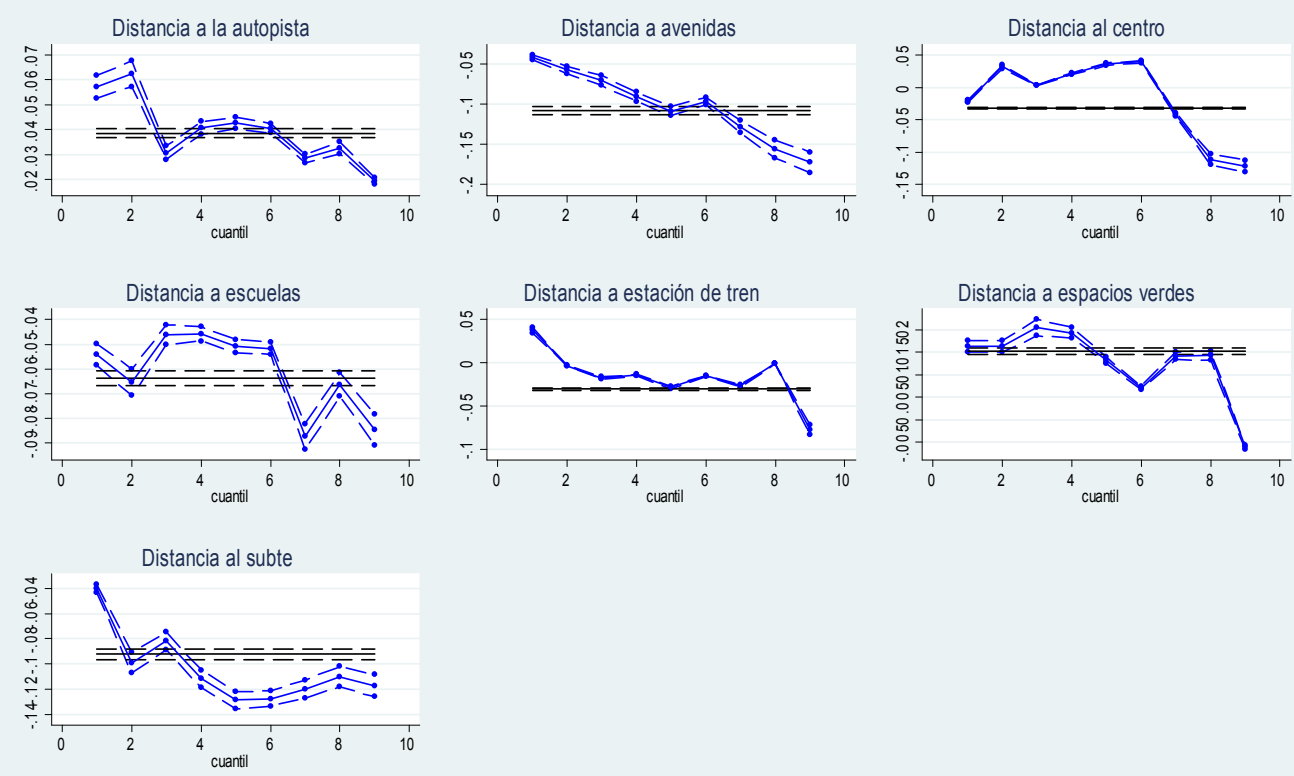

\begin{tabular}{|ll|}
\hline$-\rightarrow-$ Spatial Lag -95 & $-\rightarrow--$ Spatial Lag +95 \\
--- Cuantil -95 & --- Cuantil +95 \\
- Spatial Lag & $\longrightarrow$ Cuantil \\
\hline
\end{tabular}


Figura 4.12: Lotes, efectos por cuantil: Variables socioeconómicas
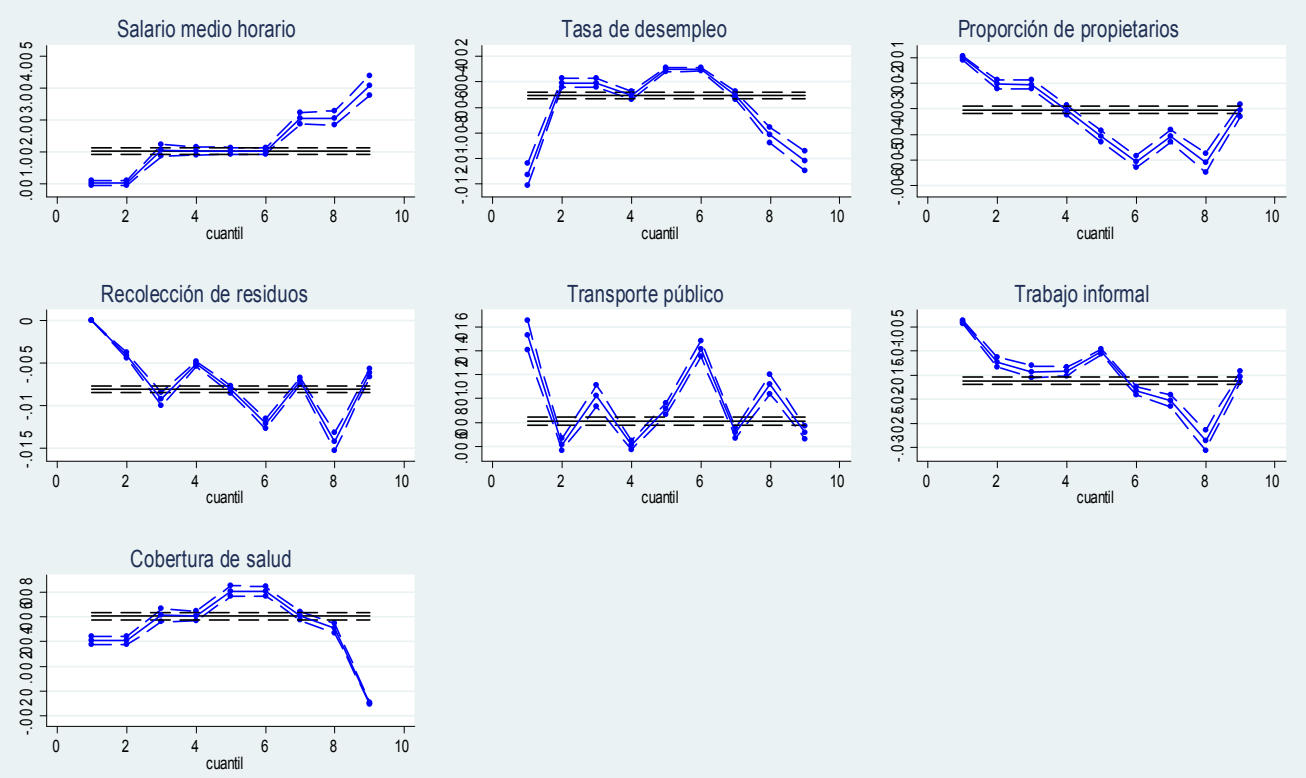

\begin{tabular}{|c|c|c|}
\hline \multirow{2}{*}{\multicolumn{2}{|c|}{ - $\rightarrow--$ Spatial Lag -95}} & \\
\hline & & - - Spatial Lag +95 \\
\hline & Cuantil -95 & Cuantil +95 \\
\hline & Spatial Lag & $\longrightarrow$ \\
\hline
\end{tabular}




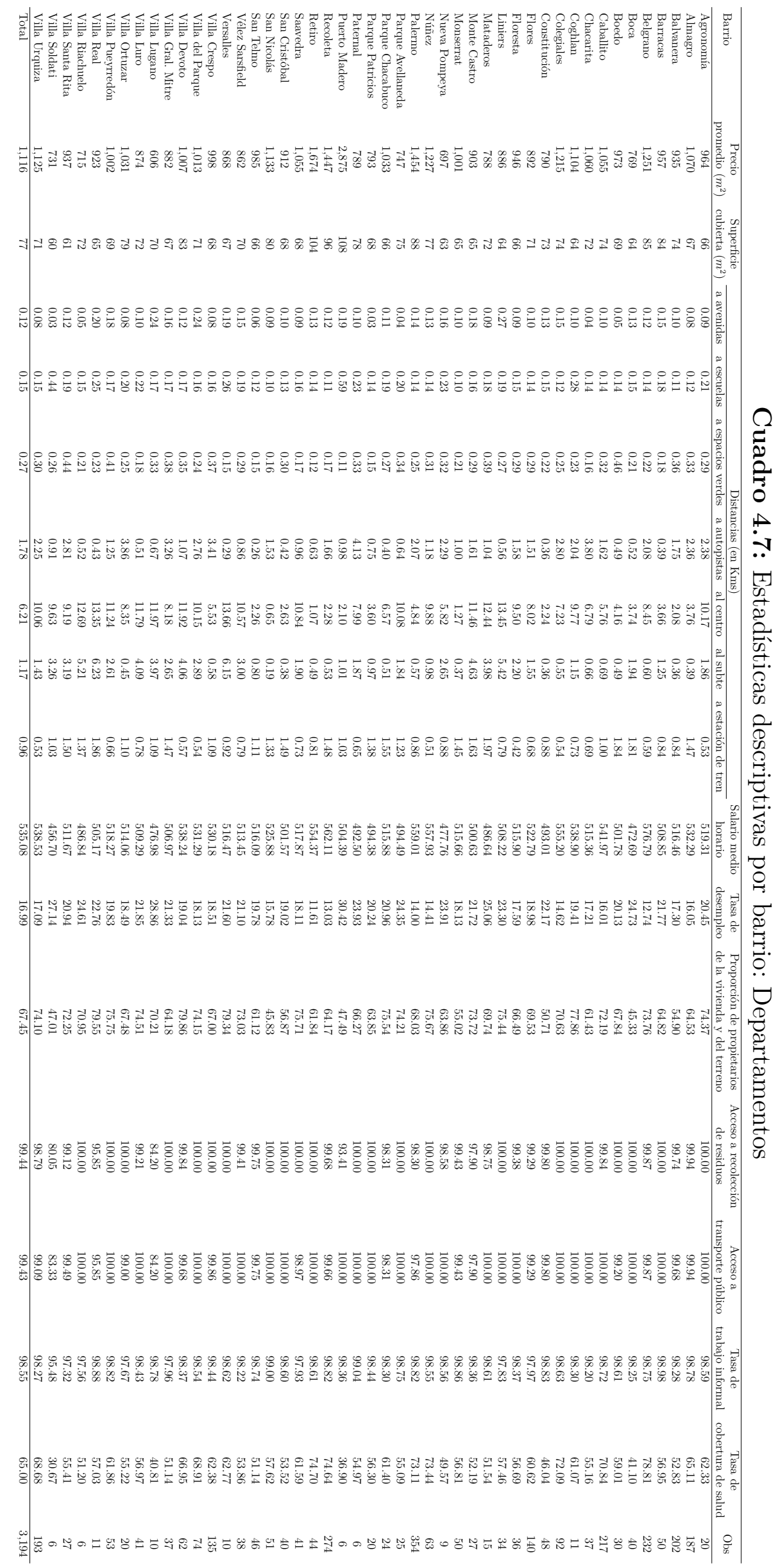




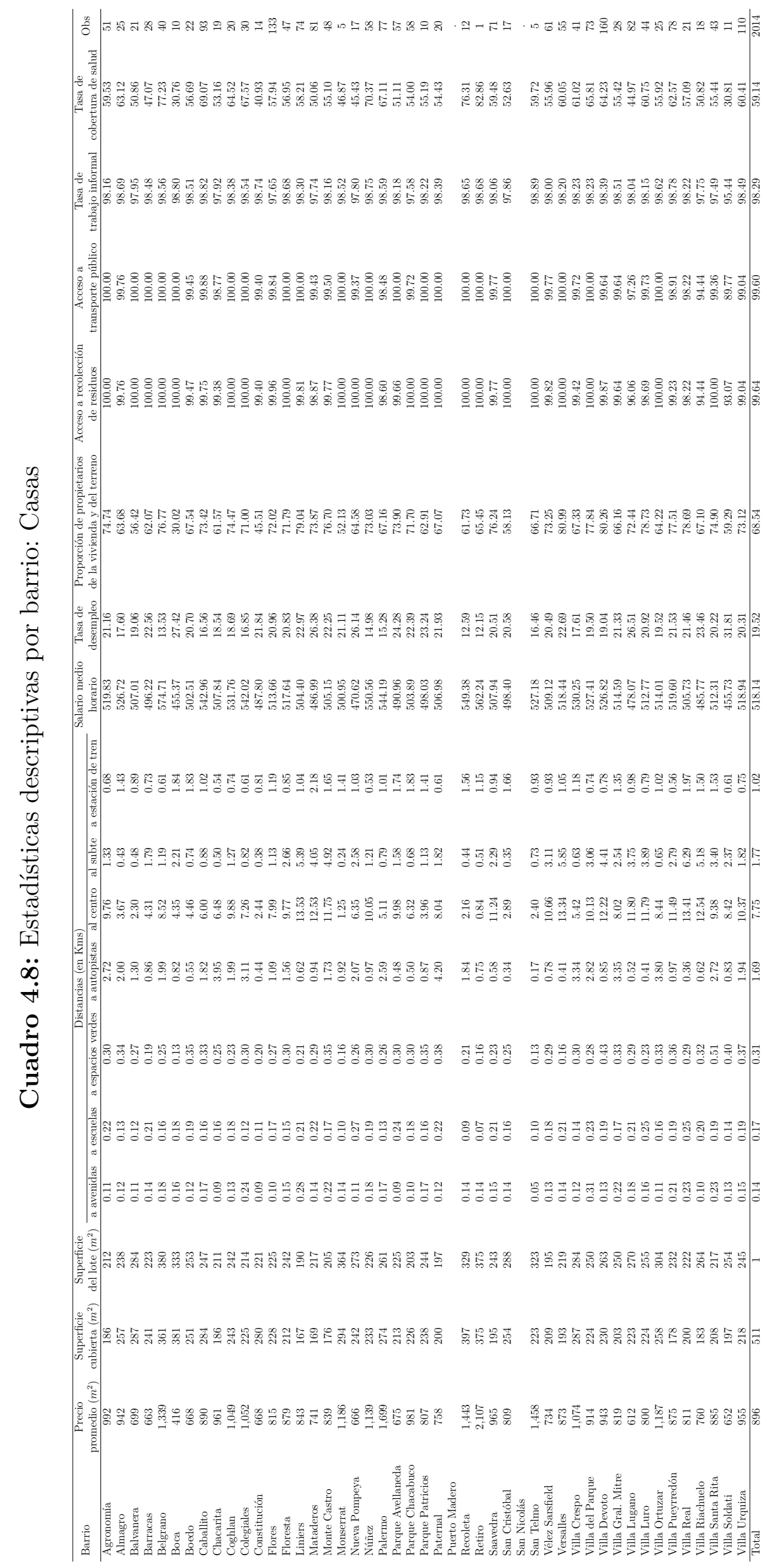




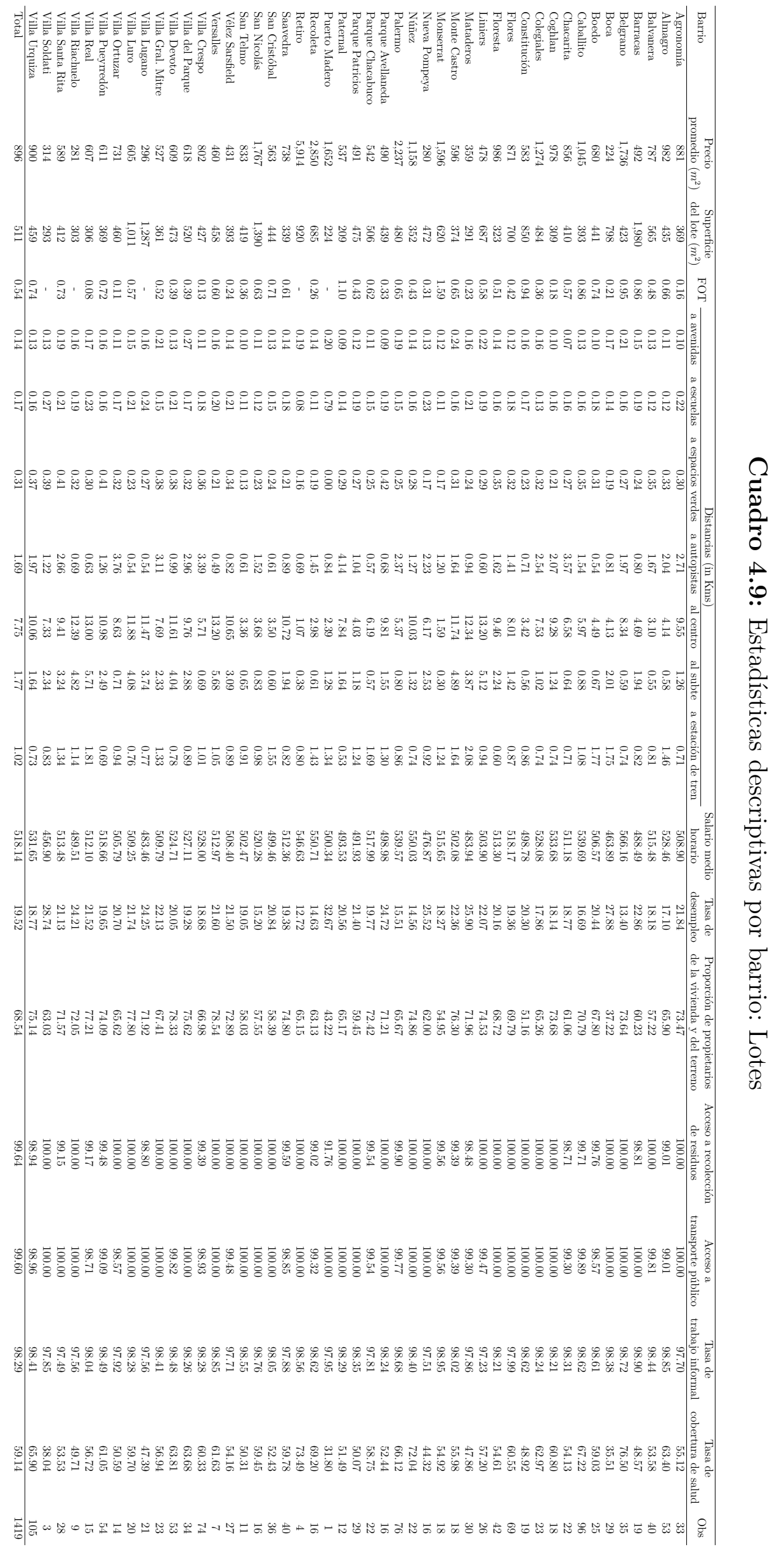




\section{Consideraciones finales}

Esta tesis doctoral aborda varios desafíos de investigación relacionados con el mercado inmobiliario. La principal motivación de esta tesis es contribuir a una mejor medición de la valuación que hacen los individuos de algunas características ambientales, tales como los riesgos de inundación y los cambios en la contaminación acústica, como así también analizar la posibilidad de que diversas características de los inmuebles puedan ser valoradas de una manera distinta a través de la distribución de precios. En algunos de los temas analizados, existe una amplia literatura empírica. Sin embargo, se centra casi por completo en los países desarrollados, mientras que la evidencia de los países en desarrollo es escasa, particularmente para Argentina casi no se observan estudios sobre dichos temas.

Una característica común a estos temas de investigación, es la inexistencia de un mercado para cada uno de estos atributos. La ausencia de un mercado explícito para cada uno de los atributos hace que la asignación de un valor a los mismos sea una tarea difícil, ya que este valor no se captura en las transacciones que se realizan regularmente en el mercado. En esta tesis se utiliza el enfoque de preferencias reveladas del que deriva el modelo de precios hedónicos. Estos modelos permiten el estudio del mercado inmobiliario y del comportamiento del consumidor mediante el análisis de los precios de los inmuebles y sus características. Si bien los modelos hedónicos tienen una larga historia, como herramientas para la valoración económica de los bienes que no tienen un mercado particular, sólo recientemente han introducido la dimensión espacial. En esta tesis se hizo una inclusión explícita de espacio en los métodos de estimación utilizando modelos hedónicos espaciales y junto con la utilización del estimador HAC (heteroskedasticity autocorrelation consistent) desarrollado por Kelejian \& Prucha (2007).

Como se describió en el capitulo metodológico, al no tener en cuenta la dependencia espacial, la heterogeneidad espacial, como así tampoco la heterocedasticidad de forma no especificada en los residuos, las estimaciones clásicas pueden llevar a conclusiones erróneas. Mejores estimaciones de los errores estándar obtenidos a través del estimador HAC proporcionan intervalos de confianza más realistas. Por otra parte, la determinación de la precisión de las estimaciones proporcionará información adicional a los responsables de las políticas públicas y ayudará a evitar la toma de decisiones incorrectas. La revelación de la verdadera precisión de las estimaciones es importante para evaluar el grado de fiabilidad de las estimaciones obtenidas. Una valoración precisa de los beneficios asociados con una política particular es especialmente relevante en el diseño, ya 
que determina si los beneficios de las políticas diseñadas exceden los costos asociados con su implementación.

Las valoraciones obtenidas en esta tesis servirán para mejorar la eficacia de las políticas públicas, las cuales tienen como objetivo mejorar la calidad de vida de las personas, como son la legislación sobre el uso del suelo, el planeamiento urbano y la aplicación de regulaciones sobre la contaminación ambiental. Es importante remarcar que uno de los aspectos más relevantes en la evaluación de dichas políticas es contar con una medida cuantitativa apropiada del valor económico de los beneficios devengados de esas políticas con los cuales contrastar los costos de las mismas (Freeman III, 2003), y es en ese punto donde esta tesis realiza su principal contribución.

La primera línea de investigación de esta tesis se relaciona con el hecho de que luego de la inundación de 2013 en la ciudad de La Plata, las autoridades empezaran a considerar la construcción de una nueva infraestructura hidráulica, a desarrollar planes de contingencia y a regular mejor el crecimiento urbanístico de la ciudad. Si bien estas políticas son necesarias, poco se sabía de los beneficios que las personas ven en este tipo de obras. Los resultados de esta tesis indican que cuando las propiedades se encuentran en zonas con riesgo hídrico sufren un descuento importante en el precio. Las estimaciones muestran que los lotes en zonas propensas a inundaciones son aproximadamente un $12 \%$ más baratos en la ciudad de La Plata. Por lo que una política destinada a proporcionar información a los potenciales compradores sobre la exposición al riesgo, sin duda aumentaría el bienestar general. La disponibilidad estimada a pagar debe ser confrontada con los costos esperados de los distintos proyectos de control de inundaciones con el fin de priorizar las inversiones futuras.

El segundo tópico está relacionado con uno de los principales problemas de los individuos que habitan las ciudades: la contaminación acústica. Un nivel de ruido por encima del umbral tolerable puede generar trastornos del sueño y del aprendizaje, de la memoria, de la motivación, en la resolución de problemas y en el incremento de la irritabilidad y de la agresividad. Muchas ciudades se ven afectadas por los altos niveles de contaminación del aire y de ruido derivados, fundamentalmente, del tráfico, de la actividad industrial y de las operaciones relacionadas con la construcción. Las estimaciones obtenidas sugieren que cuando las propiedades se encuentran en zonas más ruidosas sufren un descuento en el precio. Dicho descuento es de aproximadamente un 1,9\% en el precio del inmueble por cada decibel en la ciudad de La Plata. Adicionalmente, se analizó la sensibilidad de los costos, tanto económicos como en términos de precisión, de trabajar con mapas de ruido con menor precisión, un tema relevante para los organismos públicos que estén evaluando realizar un mapa de este tipo. El análisis de sensibilidad muestra que no hay grandes pérdidas de eficiencia si se realiza un análisis con menor cantidad de puntos de medición, siempre y cuando los puntos de medición sean cuidadosamente seleccionados.

La tercera línea de investigación estima modelos espaciales por cuantil para analizar el hecho de que algunas características de los inmuebles puedan ser valoradas de manera distinta a lo largo 
de distribución de los precios. Al contar con una amplia muestra de casas, de departamentos y de terrenos baldíos, se logró abarcar buena parte del mercado inmobiliario de la ciudad de Buenos Aires. Los resultados obtenidos muestran que los efectos de las distintas características de una propiedad sobre el precio de venta pueden ser mejor explicados por la estimación de regresiones por cuantil espaciales. El trabajo muestra la existencia de diferentes comportamientos de la dependencia espacial en los distintos tipos de propiedades y que dicha dependencia no presenta variaciones importantes entre cuantiles. Sin embargo, los efectos marginales de algunas variables se comportan de manera diferente en los distintos niveles de precios, lo cual permite concluir que los compradores de propiedades de alto precio parecen dispuestos a pagar un precio distinto por ciertas características de las propiedades que los compradores de propiedades de menor precio. Es importante señalar que las valoraciones estimadas no incluyen todos los beneficios asociados con los posibles cambios en los temas analizados. Las estimaciones muestran sólo lo que es capitalizado por los individuos en los precios de sus inmuebles. Los modelos hedónicos sólo captarán los beneficios de los cambios en los atributos que el consumidor realmente puede percibir; por lo que, si algún cambio en un atributo no es percibido por el individuo, el efecto de ese cambio no será incorporado en las medidas de bienestar ni en el precio del inmueble.

Un comentario final que resulta del análisis realizado tiene que ver con la diferente naturaleza espacial de los datos. En la primera línea de investigación, relacionada con la valoración que hacen los individuos sobre evitar los riesgos de una inundación en la ciudad de La Plata, los datos presentan tanto dependencia espacial como así también heterogeneidad espacial. Es decir, los precios o las características de los inmuebles parecen asumir valores similares en unidades geográficamente cercanas y, también, muestran la presencia de diferencias sistemáticas en el comportamiento de las relaciones bajo estudio a través del espacio. En cambio, en el análisis de la contaminación acústica, la dependencia espacial no parece estar presente en el casco fundacional de la ciudad de La Plata; Sin embargo, sí se encuentra evidencia de heterogeneidad espacial. El análisis de los datos de la ciudad de Buenos Aires muestra un comportamiento diferente a los mercados anteriores, la naturaleza espacial de los datos parece contener dependencia espacial, pero no heterogeneidad espacial. 



\section{Bibliografía}

Angeletti, K. (2000). Valuación de la contaminación del aire y del ruido por el método de los precios hedónicos: una aplicación a la ciudad de la plata. Maestría en Finanzas Públicas Provinciales y Municipales, UNLP, Agosto 2000.

Anselin, L. (1988). Spatial Econometrics: Method and Models. Dordrecht: Kluwer Academic Publishers.

Anselin, L. (1992). Spatial data analysis with gis: An introduction to application in the social sciences. National Center for Geographic Information and Analysis, University of California, Santa Barbara.

Anselin, L. (2005). Exploring spatial data with geoda: A workbook. Department of Geography, University of Illinois, Urbana-Champaign.

Anselin, L. \& Bera, A. (1998). Spatial dependence in linear regression models with an introduction to spatial econometrics. In A. Ullah \& D. Giles (Eds.), Handbook of Applied Economic Statistics, volume 155 (pp. 237-290). Marcel Lekker, New York.

Anselin, L., Bera, A., Florax, R., \& Yoon, M. (1996). Simple diagnostic tests for spatial dependence. Regional science and urban economics, 26(1), 77-104.

Anselin, L. \& Le Gallo, J. (2006). Interpolation of air quality measures in hedonic house price models: Spatial aspects. Spatial Economic Analysis, 1(1).

Arbia, G. (2006). Spatial econometrics: statistical foundations and applications to regional convergence. Berlin: Springer-Verlag.

Arraiz, I., Drukker, D., Kelejian, H., \& Prucha, I. (2010). A spatial cliff-ord-type model with heteroskedastic innovations: Small and large sample results. Journal of Regional Science, $50(2), 592-614$.

Bartosova, A., Clark, D., Novotny, V., \& Taylor, K. (2000). Using gis to evaluate the effects of flood risk on residential property values. In EPA Conference.

Bateman, I., Day, B., Lake, I., \& Lovett, A. (2000). The effect of road traffic on residential property values: A literature review and hedonic pricing study. Report to The Scottish Office, Development Department, Edinburgh, School of Environmental Sciences, University of East Anglia, Norwich. 
Bertrand, N. F. (1997). Meta-analysis of studies of willingness to pay to reduce traffic noise. MSc dissertation, University College London.

Bin, O. \& Kruse, J. (2006). Real estate market response to coastal flood hazards. Natural Hazards Review.

Bin, O., Kruse, J., \& Landry, C. (2008). Flood hazards, insurance rates, and amenities: evidence from the coastal housing market. Journal of Risk and Insurance.

Bin, O. \& Polasky, S. (2004). Effects of flood hazards on property values: Evidence before and after hurricane floyd. Land Economics, 80(4), 490-500.

BMRBB, B. M. R. B. (2005). Environment agency launches campaign to tackle flood apathy. disponible en www.marketresearchworld.net.

Burningham, K., Fielding, J., \& Thrush, D. (2008). It will never happen to me: understanding public awareness of local flood risk. Disasters, 32(2), $216-238$.

Burridge, P. (1980). On the cliff-ord test for spatial correlation. Journal of the Royal Statistical Society, Series B Methodological, (pp. 107-108).

Buse, A. (1973). Goodness of fit in generalized least squares estimation. The American Statistician, 27(3), 106-108.

Buse, A. (1979). Goodness-of-fit in the seemingly unrelated regressions model: a generalization. Journal of Econometrics, 10(1), 109-113.

Carbone, J., Hallstrom, D., \& Smith, K. (2006). Can natural experiments measure behavioral responses to environmental risks? Environmental and Resource Economics, 33(3), 273-97.

Case, K. \& Shiller, R. (2003). Is there a bubble in the housing market? Brookings Papers on Economic Activity, 2, 299-342.

Chernozhukovm, V. \& Hansen, C. (2006). Instrumental quantile regression inference for structural and treatment effect models. Journal of Econometrics, 127, 491-525.

Chivers, J. \& Flores, N. E. (2002). Market failure in information: the national flood insurance program. Land Economics, 78(4), 515 - 521.

Conte Grand, M. (2001). Una primera aproximación a la valuación hedónica de la contaminación en buenos aires. Serie Documentos de Trabajo, Universidad del CEMA: Área: economía, No. $20 \%$.

Cruces, G., Fernández, G., \& Ham, A. (2007). There goes the neighborhood: Urban quality of life and real estate prices in buenos aires. CEDLAS Working Paper.

Daniel, V., Florax, R., \& Rietveld, P. (2009). Flooding risk and housing values: An economic assessment of environmental hazard. Ecological Economics, 69, 355-365. 
Day, B. (2001). The theory of hedonic markets: Obtaining welfare measures for changes in environmental quality using hedonic market data. Economics for the Environment Consultancy (Eftec), London.

Day, B. (2003). Submarket identification in property markets: a hedonic housing price model for Glasgow. Technical report, CSERGE Working Paper EDM.

DEFRA (2008). Abi/government statement on flooding and insurance for england (london: Defra).

Diem, J. (2003). A critical examination of ozone mapping from a spatial-scale perspective. Environmental pollution, 125(3), 369-383.

Donnelly, W. (1998). Hedonic price analysis of the effect of a floodplain on property values. Journal of the American Water Resources Association, 25(3), 581-586.

Drukker, D., Egger, P., \& Prucha, I. (2013). On two-step estimation of a spatial autoregressive model with autoregressive disturbances and endogenous regressors. Econometric Reviews, 32(5-6), 686-733.

Florax, R. \& Van der Vlist, A. (2003). Spatial econometric data analysis: Moving beyond traditional models. International Regional Science Review, 26(3), 223-243.

Freeman III, M. (1979). Hedonic prices, property values and measuring environmental benefits: a survey of the issues. The Scandinavian Journal of Economics, (pp. 154-173).

Freeman III, M. (2003). Economic valuation: what and why. In P. Champ, K. Boyle, \& T. Brown (Eds.), A primer on nonmarket valuation, volume 3 chapter 1, (pp. 1-25). Springer.

Fujita, M., Krugman, P., \& Venables, A. (1999). The Spatial Economics: Cities, Regions and International Trade. Cambridge, MA: MIT Press.

Geary, R. (1954). The contiguity ratio and statistical mapping. The incorporated statistician, (pp. 115-146).

Gibbons, S. \& Overman, H. (2012). Mostly pointless spatial econometrics. Journal of Regional Science, 52(2), 172-191.

Griffith, D. (1996). Some guidelines for specifying the geographic weights matrix contained in spatial statistical models. Practical handbook of spatial statistics, (pp. $65-82$ ).

Griliches, Z. (1961). Hedonic price indexes for automobiles: An econometric of quality change. In The Price Statistics of the Federal Goverment (pp. 173-196). NBER.

Hallegatte, S., Green, C., Nicholls, R., \& Corfee-Morlot, J. (2013). Future flood losses in major coastal cities. Nature Climate Change, 3, 802-806.

Harrison, D. \& Rubinfeld, D. (1978). Hedonic housing prices and the demand for clean air. Journal of environmental economics and management, 5(1), 81-102. 
Harrison, D., Smersh, G., \& Schwartz, A. (2001). Environmental determinants of housing prices: The impact of flood zone status. Journal of Real Estate Research, 21(1-2), 3-20.

Hawkins, R. (1999). Review of studies on external costs of noise. prepared. Environment Protection Economics Division, DETR (DEFRA).

Herrera, M. (2015). Econometría espacial usando stata. breve guía aplicada para datos de corte transversal. Documentos de Trabajo del IELDE, (13). Documento Técnico No 1.

Herrera, M., Mur, J., \& Ruiz, M. (2014). Detecting causal relationships between spatial processes. Papers in Regional Science. Doi: 10.1111/pirs.12144.

Hurtado, M., Giménez, J., Cabral, M., Silva, M., Martinez, O., Camilion, M., Sanchez, C., Gebhard, J., Forte, L., Boff, L., Crincoli, A., \& Lucesoli, H. (2006). Analisis ambiental del partido de La Plata: Aportes al ordenamiento territorial. Consejo Federal de Inversiones, Facultad de Ciencias Naturales y Museo, La Plata.

IFRC (2014). World disasters report 2014. Geneva: International Federation of Red Cross 6 Red Crescent Societies.

IPCC (2014). Summary for policymakers. In: Climate Change 2014: Impacts, Adaptation, and Vulnerability. Part A: Global and Sectoral Aspects. Contribution of Working Group II to the Fifth Assessment Report of the Intergovernmental Panel on Climate Change. Cambridge University Press, Cambridge, United Kingdom and New York, NY, USA.

Kelejian, H. \& Prucha, I. (1998). A generalized spatial two-stage least squares procedure for estimating a spatial autoregressive model with autoregressive disturbances. The Journal of Real Estate Finance and Economics, 17(1), 99-121.

Kelejian, H. \& Prucha, I. (1999). A generalized moments estimator for the autoregressive parameter in a spatial model. International economic review, 40(2), 509-533.

Kelejian, H. \& Prucha, I. (2007). Hac estimation in a spatial framework. Journal of Econometrics, 140, 131-154.

Kelejian, H. \& Prucha, I. (2010). Specification and estimation of spatial autoregressive models with autoregressive and heteroskedastic disturbances. Journal of Econometrics, 157(1), 5367.

Kim, C., Phipps, T., \& Anselin, L. (2003). Measuring the benefits of air quality improvement: a spatial hedonic approach. Journal of environmental economics and management, 45(1), 24-39.

Kim, T.-H. \& Muller, C. (2004). Two-stage quantile regression when the first stage is based on quantile regression. Econometric Journal, 7, 218-231.

Koenker, R. (2005). Quantile Regression. Cambridge University Press.

Koenker, R. \& Bassett, G. S. (1978). Regression quantiles. Econometrica, (46), 33-50. 
Koenker, R. \& Hallock, K. (2001). Quantile regression. Journal of Economic Perspective, 14, $143-156$.

Kostov, P. (2009). A spatial quantile regression hedonic model of agriculture land prices. Spatial Economic Analysis, 4, 53-72.

Kousky, C. (2010). Learning from extreme events: Risk perceptions after the flood. Land Economics, 86.(3), 395-422.

Krugman, P. (1991). Geography and trade. Cambridge, MIT Press.

Lancaster, K. (1966). A new approach to consumer theory. Journal of Political Economy, 74(1), $132-157$.

Lee, L.-f. (2003). Best spatial two-stage least squares estimators for a spatial autoregressive model with autoregressive disturbances. Econometric Reviews, 22(4), 307-335.

LeSage, J. \& Pace, K. (2009). Introduction to Spatial Econometrics. CRC Press Taylor \& Francis Group.

Liao, W.-C. \& Wang, X. (2012). Hedonic house prices and spatial quantile regression. Journal of Housing Economic, 21, 16-27.

Mak, S., Choy, L., \& Ho, W. (2010). Quantile regression estimates of hong kong real estate prices. Urban Studies, 47(11), $2461-247$.

Malpezzi, S. (2003). Hedonic pricing models: a selective and applied review. Section in Housing Economics and Public Policy: Essays in Honor of Duncan Maclennan.

McMillen, D. (2008). Changes in the distribution of house prices over time: Structural characteristics, neighborhood, or coefficients? Journal of Urban Economics, 64, 573 - 589.

McMillen, D. \& Thorsnes, P. (2006). Housing renovations and the quantile repeat-sales price index. Real Estate Economics, 34(4), 567 - 584.

Moran, P. A. P. (1948). The interpretation of statistical maps. Journal of Royal Statistical Society.

Muellbauer, J. \& Murphy, A. (2008). Housing markets and the economy: the assessment. Oxford review of economic policy, 24(1), 1-33.

Navrud, S. (2002). The state of the art on economic valuation of noise. Final Report to European Commission DG Environment.

Nelson, J. (2004). Meta-analysis of airport noise and hedonic property values. Journal of Transport Economics and Policy, London School of Economics and University of Bath, 38(1), $1-27$.

Park, W. \& Miller, W. (1982). Flood risk perceptions and overdevelopment in the floodplain. Journal of the American Water Resources Association, 18(1), 89-94. 
Rambaldi, A., Fletcher, C., Collins, K., \& McAllister, R. (2013). Housing shadow prices in an inundation-prone suburb. Urban Studies, 50(9), 1889-1905.

Ridker, R. \& Henning, J. (1967). The determinants of residential property values with special reference to air pollution. The Review of Economics and Statistics, (pp. 246-257).

Rosen, S. (1974). Hedonic prices and implicit markets: product differentiation in pure competition. Journal of Political Economy, 1(82), 34-55.

Samarasinghe, O. \& Sharp, B. (2010). Flood prone risk and amenity values: a spatial hedonic analysis. Australian Journal of Agricultural and Resource Economics, 54(4), 457-475.

Sheppard, S. (1999). Hedonic analysis of housing markets. In P. Cheshire (Ed.), Handbook of Urban and Regional Economics, volume 3 (pp. 1595-1635). Elsevier.

Speyrer, J. \& Ragas, W. (1991). Housing prices and flood risk: an examination using spline regression. Journal of Real Estate Finance and Economics, 4(4), 395-407.

Su, L. \& Yang, Z. (2011). Instrumental variable quantile estimation of spatial autoregressive models. Working paper.

Thompson, M. \& Stoevener, H. (1983). Estimating residential flood control benefits using implicit price equations. Journal of the American Water Resources Association, 19(6), 889896.

Tobin, G. \& Montz, B. (1994). The flood hazard and dynamics of the urban residential land market. Water Resources Bulletin, 30(4), 673-685.

Tobin, G. \& Newton, T. (1986). A theoretical framework of flood induced changes in urban land values. Water Resources Bulletin, 22(1), 67-71.

Wake, B. (2013). Flooding costs. Nature Climate Change, 3(778).

WHO (2011). Burden of disease from environmental noise. Quantification of healthy life years lost in Europe. World Health Organization.

Zhai, G., Fukuzono, T., \& Ikeda, S. (2003). Effect of flooding on megalopolitan land prices: A case study of the 2000 tokai flood in japan. Journal of Natural Disaster Science, 25(1), $23-36$.

Zietz, J., Zietz, E., \& Sirmans, S. (2008). Determinants of house prices: a quantile regression approach. Journal of Real Estate Finance and Economics, 37, 317-333. 